\title{
S1
}

Supporting Information

\section{$N$-Benzyl-Isatin Sulfonamide Analogs as Potent Caspase-3 Inhibitors: Synthesis, In Vitro Activity, and Molecular Modeling Studies}

Wenhua Chu, Jun Zhang,, Chenbo Zeng, Justin Rothfuss, Zhude Tu, Yunxiang Chu, David E. Reichert, Michael J. Welch and Robert H. Mach

\section{Table of Contents}

- Table of Elemental Analysis Data

S2

- Mole2 Files

S3-S91 
Elemental Analysis

\begin{tabular}{|c|c|c|c|c|c|c|c|}
\hline \multirow[t]{2}{*}{ Compd } & \multirow[t]{2}{*}{ Formula } & \multicolumn{3}{|c|}{ Calcd } & \multicolumn{3}{|c|}{ Found } \\
\hline & & C & $\mathrm{H}$ & $\mathrm{N}$ & C & $\mathrm{H}$ & $\mathrm{N}$ \\
\hline 10 & $\mathrm{C}_{19} \mathrm{H}_{18} \mathrm{~N}_{2} \mathrm{O}_{5} \mathrm{~S}$ & 59.06 & 4.70 & 7.25 & 58.99 & 4.74 & 7.11 \\
\hline $11 a$ & $\mathrm{C}_{20} \mathrm{H}_{20} \mathrm{~N}_{2} \mathrm{O}_{5} \mathrm{~S}$ & 59.99 & 5.03 & 7.00 & 59.80 & 5.03 & 6.91 \\
\hline 2 & $\mathrm{C}_{26} \mathrm{H}_{24} \mathrm{~N}_{2} \mathrm{O}_{5} \mathrm{~S}$ & 65.53 & 5.08 & 5.88 & 65.27 & 5.32 & 5.58 \\
\hline $11 b$ & $\mathrm{C}_{27} \mathrm{H}_{26} \mathrm{~N}_{2} \mathrm{O}_{6} \mathrm{~S}$ & 64.02 & 5.17 & 5.53 & 64.76 & 5.24 & 5.06 \\
\hline $11 \mathrm{c}$ & $\mathrm{C}_{26} \mathrm{H}_{23} \mathrm{FN}_{2} \mathrm{O}_{5} \mathrm{~S}$ & 63.15 & 4.69 & 5.66 & 63.05 & 4.69 & 5.60 \\
\hline 11d & $\mathrm{C}_{27} \mathrm{H}_{26} \mathrm{~N}_{2} \mathrm{O}_{5} \mathrm{~S}_{2}$ & 62.05 & 5.01 & 5.36 & 61.81 & 4.95 & 5.34 \\
\hline $11 e$ & $\mathrm{C}_{28} \mathrm{H}_{26} \mathrm{~N}_{2} \mathrm{O}_{7} \mathrm{~S}$ & 62.91 & 4.90 & 5.24 & 62.99 & 5.02 & 5.13 \\
\hline $11 f$ & $\mathrm{C}_{26} \mathrm{H}_{24} \mathrm{~N}_{2} \mathrm{O}_{6} \mathrm{~S} \cdot 0.25 \mathrm{H}_{2} \mathrm{O}$ & 62.83 & 4.97 & 5.64 & 62.87 & 4.74 & 5.69 \\
\hline $11 \mathrm{~g}$ & $\mathrm{C}_{25} \mathrm{H}_{22} \mathrm{FN}_{3} \mathrm{O}_{5} \mathrm{~S}$ & 60.60 & 4.47 & 8.48 & 60.60 & 4.59 & 8.33 \\
\hline $11 \mathrm{~h}$ & $\mathrm{C}_{25} \mathrm{H}_{22} \mathrm{FN}_{3} \mathrm{O}_{5} \mathrm{~S}$ & 60.60 & 4.47 & 8.48 & 60.32 & 4.34 & 8.35 \\
\hline $11 \mathrm{i}$ & $\mathrm{C}_{25} \mathrm{H}_{22} \mathrm{FN}_{3} \mathrm{O}_{5} \mathrm{~S} \cdot 0.25 \mathrm{H}_{2} \mathrm{O}$ & 60.05 & 4.54 & 8.40 & 60.06 & 4.49 & 8.24 \\
\hline 17 & $\mathrm{C}_{18} \mathrm{H}_{16} \mathrm{~N}_{2} \mathrm{O}_{5} \mathrm{~S} \cdot 0.5 \mathrm{H}_{2} \mathrm{O}$ & 56.68 & 4.49 & 7.34 & 56.96 & 4.39 & 7.30 \\
\hline $18 a$ & $\mathrm{C}_{19} \mathrm{H}_{18} \mathrm{~N}_{2} \mathrm{O}_{5} \mathrm{~S}$ & 59.06 & 4.70 & 7.25 & 58.98 & 4.75 & 7.19 \\
\hline $18 b$ & $\mathrm{C}_{25} \mathrm{H}_{22} \mathrm{~N}_{2} \mathrm{O}_{5} \mathrm{~S}$ & 64.92 & 4.79 & 6.06 & 64.82 & 4.79 & 5.97 \\
\hline $18 c$ & $\mathrm{C}_{26} \mathrm{H}_{24} \mathrm{~N}_{2} \mathrm{O}_{6} \mathrm{~S}$ & 63.40 & 4.91 & 5.69 & 63.65 & 4.93 & 5.59 \\
\hline $18 d$ & $\mathrm{C}_{26} \mathrm{H}_{24} \mathrm{~N}_{2} \mathrm{O}_{5} \mathrm{~S}_{2}$ & 61.40 & 4.76 & 5.51 & 60.99 & 4.71 & 5.36 \\
\hline $18 e$ & $\mathrm{C}_{25} \mathrm{H}_{21} \mathrm{FN}_{2} \mathrm{O}_{5} \mathrm{~S}$ & 62.49 & 4.41 & 5.83 & 62.27 & 4.48 & 5.69 \\
\hline $18 f$ & $\mathrm{C}_{25} \mathrm{H}_{21} \mathrm{FN}_{2} \mathrm{O}_{5} \mathrm{~S}$ & 62.49 & 4.41 & 5.83 & 62.25 & 4.47 & 5.68 \\
\hline $18 \mathrm{~g}$ & $\mathrm{C}_{24} \mathrm{H}_{20} \mathrm{FN}_{3} \mathrm{O}_{5} \mathrm{~S}$ & 59.87 & 4.19 & 8.73 & 59.81 & 4.16 & 8.62 \\
\hline $18 \mathrm{~h}$ & $\mathrm{C}_{24} \mathrm{H}_{20} \mathrm{FN}_{3} \mathrm{O}_{5} \mathrm{~S} \cdot 0.5 \mathrm{H}_{2} \mathrm{O}$ & 58.87 & 4.32 & 8.57 & 58.69 & 4.45 & 8.26 \\
\hline $18 i$ & $\mathrm{C}_{24} \mathrm{H}_{20} \mathrm{FN}_{3} \mathrm{O}_{5} \mathrm{~S}$ & 59.87 & 4.19 & 8.73 & 59.59 & 4.27 & 8.48 \\
\hline 20 & $\mathrm{C}_{18} \mathrm{H}_{17} \mathrm{~N}_{3} \mathrm{O}_{5} \mathrm{~S} .0 .5 \mathrm{H}_{2} \mathrm{O}$ & 54.54 & 4.58 & 10.60 & 54.56 & 4.70 & 10.04 \\
\hline $21 a$ & $\mathrm{C}_{19} \mathrm{H}_{19} \mathrm{~N}_{3} \mathrm{O}_{5} \mathrm{~S}$ & 56.85 & 4.77 & 10.47 & 56.48 & 4.87 & 10.19 \\
\hline $21 c$ & $\mathrm{C}_{26} \mathrm{H}_{25} \mathrm{~N}_{3} \mathrm{O}_{6} \mathrm{~S}$ & 61.53 & 4.96 & 8.28 & 61.27 & 4.95 & 8.17 \\
\hline 23 & $\mathrm{C}_{18} \mathrm{H}_{17} \mathrm{~N}_{3} \mathrm{O}_{5} \mathrm{~S} \cdot 0 \cdot 75 \mathrm{H}_{2} \mathrm{O}$ & 53.92 & 4.65 & 10.48 & 54.14 & 4.39 & 10.35 \\
\hline
\end{tabular}




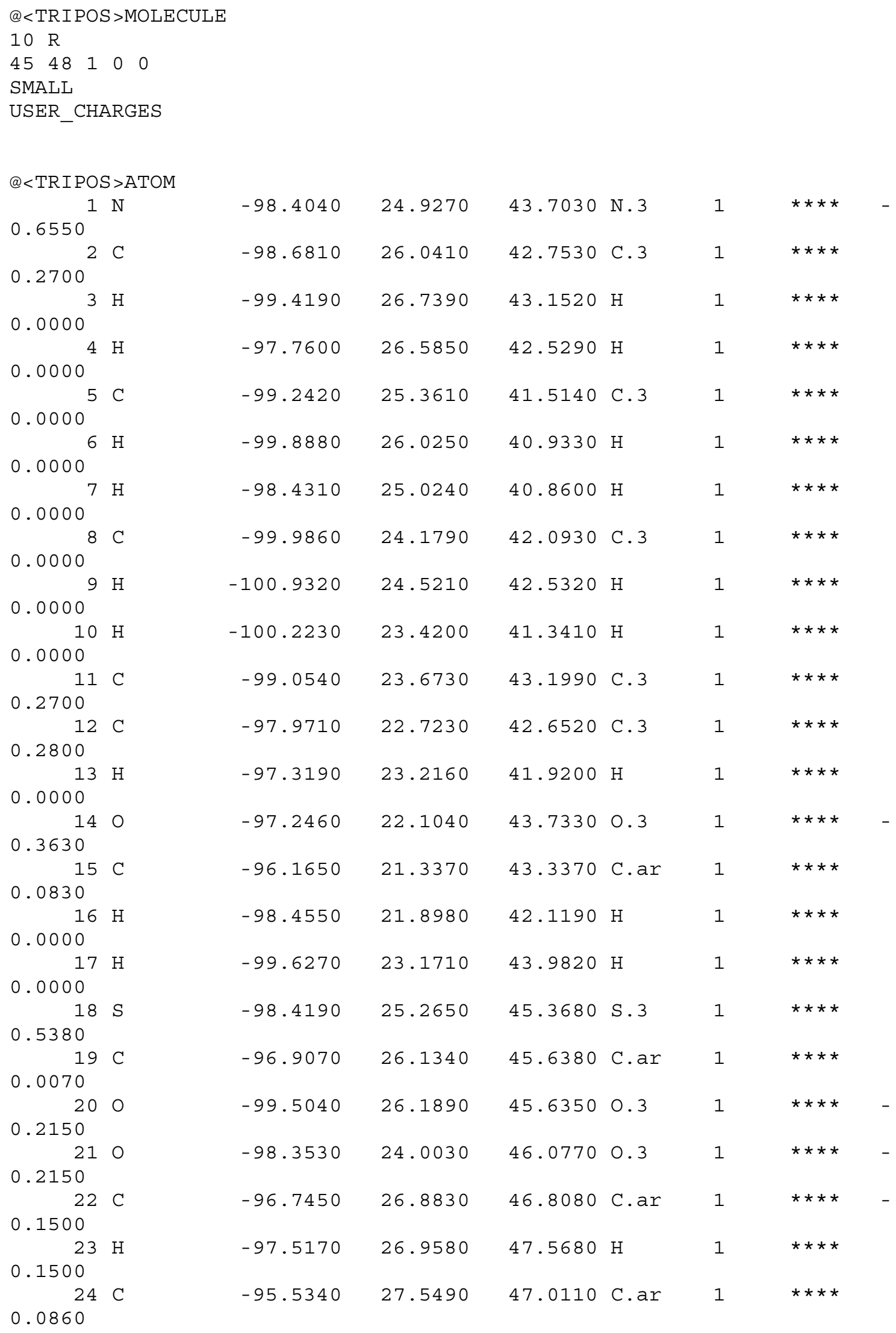




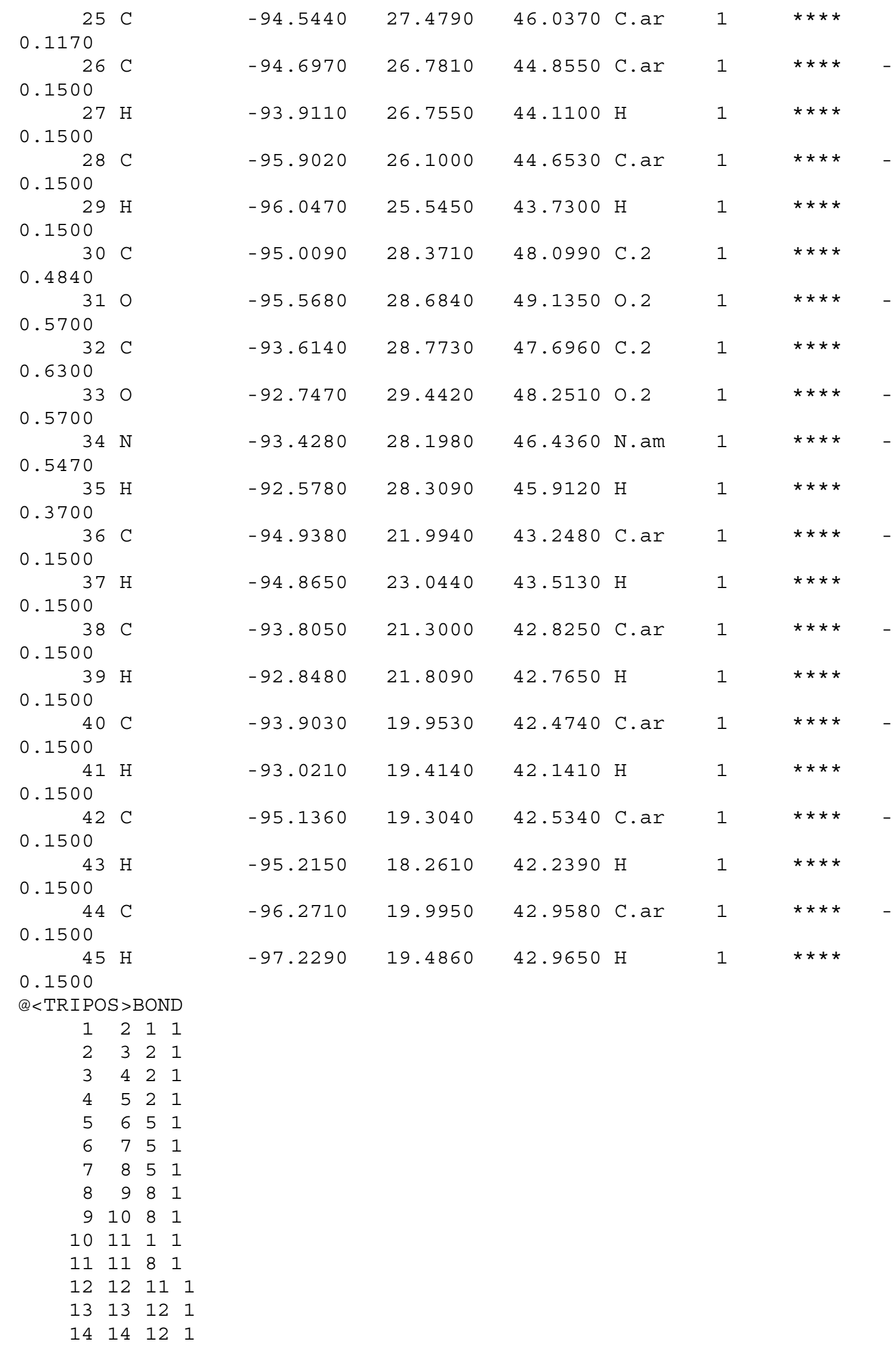




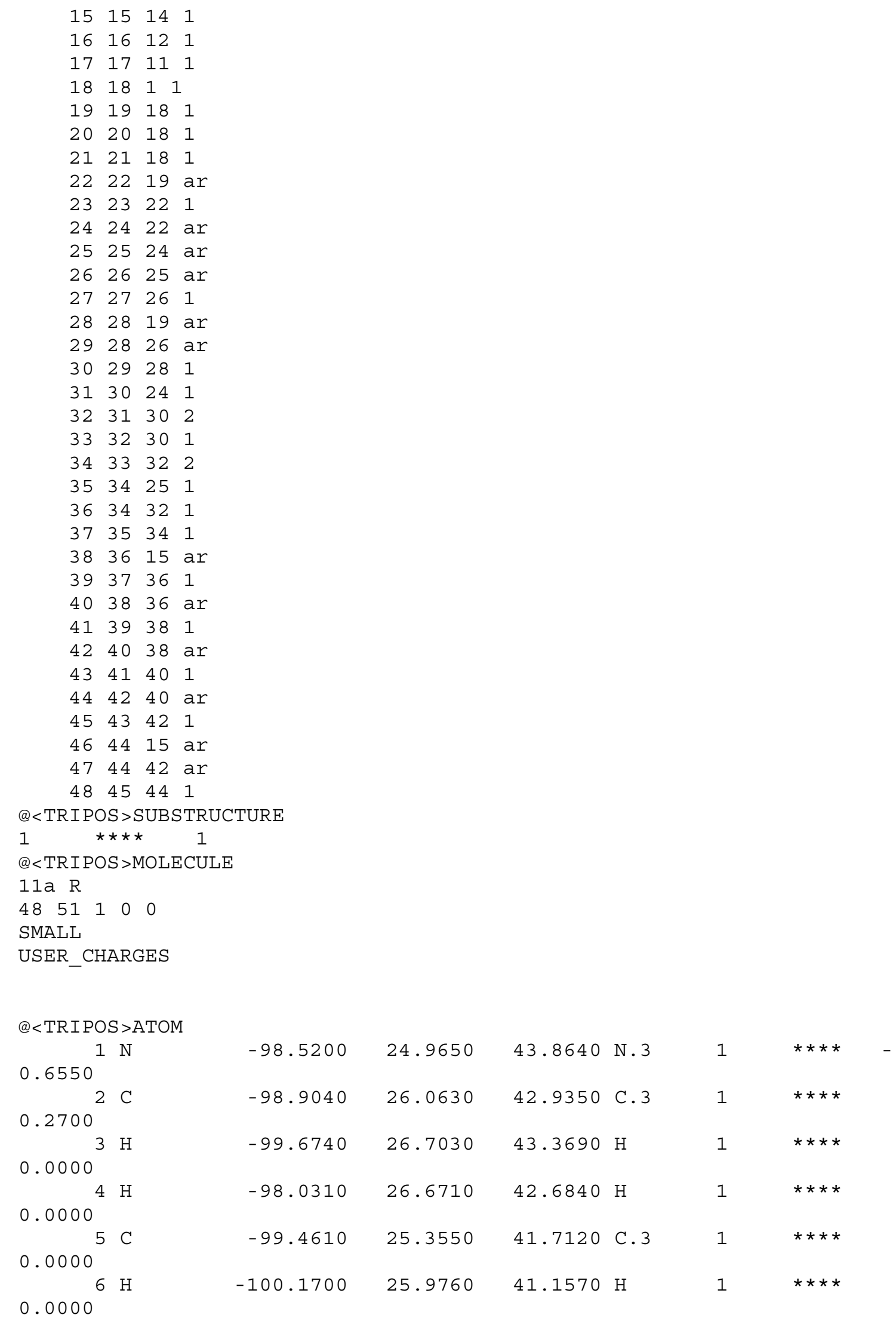




\begin{tabular}{|c|c|c|c|c|c|c|c|}
\hline 7 & $\mathrm{H}$ & -98.6500 & 25.0760 & 41.0260 & $\mathrm{H}$ & 1 & $\star \star \star \star *$ \\
\hline \multicolumn{8}{|c|}{0.0000} \\
\hline 8 & $\mathrm{C}$ & -100.1040 & 24.1210 & 42.3060 & C. 3 & 1 & $\star \star \star \star *$ \\
\hline \multicolumn{8}{|c|}{0.0000} \\
\hline 9 & $\mathrm{H}$ & -101.0540 & 24.3940 & 42.7780 & $\mathrm{H}$ & 1 & $\star \star \star \star *$ \\
\hline \multicolumn{8}{|c|}{0.0000} \\
\hline 10 & $\mathrm{H}$ & -100.3140 & 23.3530 & 41.5550 & $\mathrm{H}$ & 1 & $\star \star \star \star$ \\
\hline \multicolumn{8}{|c|}{0.0000} \\
\hline 11 & $\mathrm{C}$ & -99.1010 & 23.6730 & 43.3750 & C. 3 & 1 & $\star \star \star \star *$ \\
\hline \multicolumn{8}{|c|}{0.2700} \\
\hline 12 & $\mathrm{C}$ & -97.9810 & 22.7930 & 42.7830 & C. 3 & 1 & $\star \star \star \star *$ \\
\hline \multicolumn{8}{|c|}{0.2800} \\
\hline 13 & $\mathrm{H}$ & -97.3390 & 23.3400 & 42.0840 & $\mathrm{H}$ & 1 & $\star \star \star \star$ \\
\hline \multicolumn{8}{|c|}{0.0000} \\
\hline 14 & $\mathrm{O}$ & -97.2490 & 22.1290 & 43.8310 & 0.3 & 1 & $\star \star \star \star *$ \\
\hline \multicolumn{8}{|l|}{0.3630} \\
\hline 15 & $\mathrm{C}$ & -96.2910 & 21.2170 & 43.4230 & C.ar & 1 & $\star \star \star \star *$ \\
\hline \multicolumn{8}{|c|}{0.0830} \\
\hline 16 & $\mathrm{H}$ & -98.4390 & 21.9860 & 42.1990 & $\mathrm{H}$ & 1 & $\star \star \star \star$ \\
\hline \multicolumn{8}{|c|}{0.0000} \\
\hline 17 & $\mathrm{H}$ & -99.6140 & 23.1320 & 44.1750 & $\mathrm{H}$ & 1 & $\star \star \star \star \star$ \\
\hline \multicolumn{8}{|c|}{0.0000} \\
\hline 18 & $S$ & -98.4750 & 25.2930 & 45.5270 & S. 3 & 1 & $\star \star \star \star$ \\
\hline \multicolumn{8}{|c|}{0.5380} \\
\hline 19 & $\mathrm{C}$ & -96.9290 & 26.1190 & 45.7590 & C.ar & 1 & $\star \star \star \star \star$ \\
\hline \multicolumn{8}{|c|}{0.0070} \\
\hline 20 & O & -99.5200 & 26.2470 & 45.8420 & 0.3 & 1 & $\star \star \star \star$ \\
\hline \multicolumn{8}{|c|}{0.2150} \\
\hline 21 & O & -98.4090 & 24.0280 & 46.2300 & 0.3 & 1 & $\star \star \star \star \star$ \\
\hline \multicolumn{8}{|c|}{0.2150} \\
\hline 22 & $\mathrm{C}$ & -96.7290 & 26.8950 & 46.9050 & C.ar & 1 & $\star \star \star \star$ \\
\hline \multicolumn{8}{|c|}{0.1500} \\
\hline 23 & $\mathrm{H}$ & -97.4890 & 26.9920 & 47.6780 & $\mathrm{H}$ & 1 & $\star \star \star \star *$ \\
\hline \multicolumn{8}{|c|}{0.1500} \\
\hline 24 & $\mathrm{C}$ & -95.5020 & 27.5420 & 47.0630 & C.ar & 1 & $\star \star \star \star$ \\
\hline \multicolumn{8}{|c|}{0.0860} \\
\hline 25 & $\mathrm{C}$ & -94.5250 & 27.4390 & 46.0740 & C.ar & 1 & $\star \star \star \star \star$ \\
\hline 0.1170 & & & & & & & \\
\hline 26 & $\mathrm{C}$ & -94.7250 & 26.7040 & 44.9190 & C.ar & 1 & $\star \star \star \star$ \\
\hline 0.1500 & & & & & & & \\
\hline 27 & $\mathrm{H}$ & -93.9650 & 26.6380 & 44.1490 & $\mathrm{H}$ & 1 & $\star \star \star \star *$ \\
\hline 0.1500 & & & & & & & \\
\hline 28 & $\mathrm{C}$ & -95.9460 & 26.0410 & 44.7590 & C.ar & 1 & 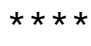 \\
\hline 0.1500 & & & & & & & \\
\hline 29 & $\mathrm{H}$ & -96.1200 & 25.4690 & 43.8500 & $\mathrm{H}$ & 1 & $\star \star \star \star *$ \\
\hline 0.1500 & & & & & & & \\
\hline 30 & $\mathrm{C}$ & -94.9670 & 28.3910 & 48.1210 & C. 2 & 1 & $\star \star \star \star *$ \\
\hline 0.4840 & & & & & & & \\
\hline 31 & O & -95.5170 & 28.7260 & 49.1540 & 0.2 & 1 & $\star \star \star \star$ \\
\hline 0.5700 & & & & & & & \\
\hline 32 & $\mathrm{C}$ & -93.5790 & 28.7970 & 47.6950 & C. 2 & 1 & $\star \star \star \star *$ \\
\hline 0.6300 & & & & & & & \\
\hline 33 & O & -92.7590 & 29.5250 & 48.2460 & 0.2 & 1 & $\star \star \star \star *$ \\
\hline 0.5700 & & & & & & & \\
\hline $\begin{array}{r}34 \\
0.4770\end{array}$ & $\mathrm{~N}$ & -93.3960 & 28.1790 & 46.4450 & N.am & 1 & 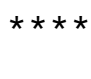 \\
\hline
\end{tabular}




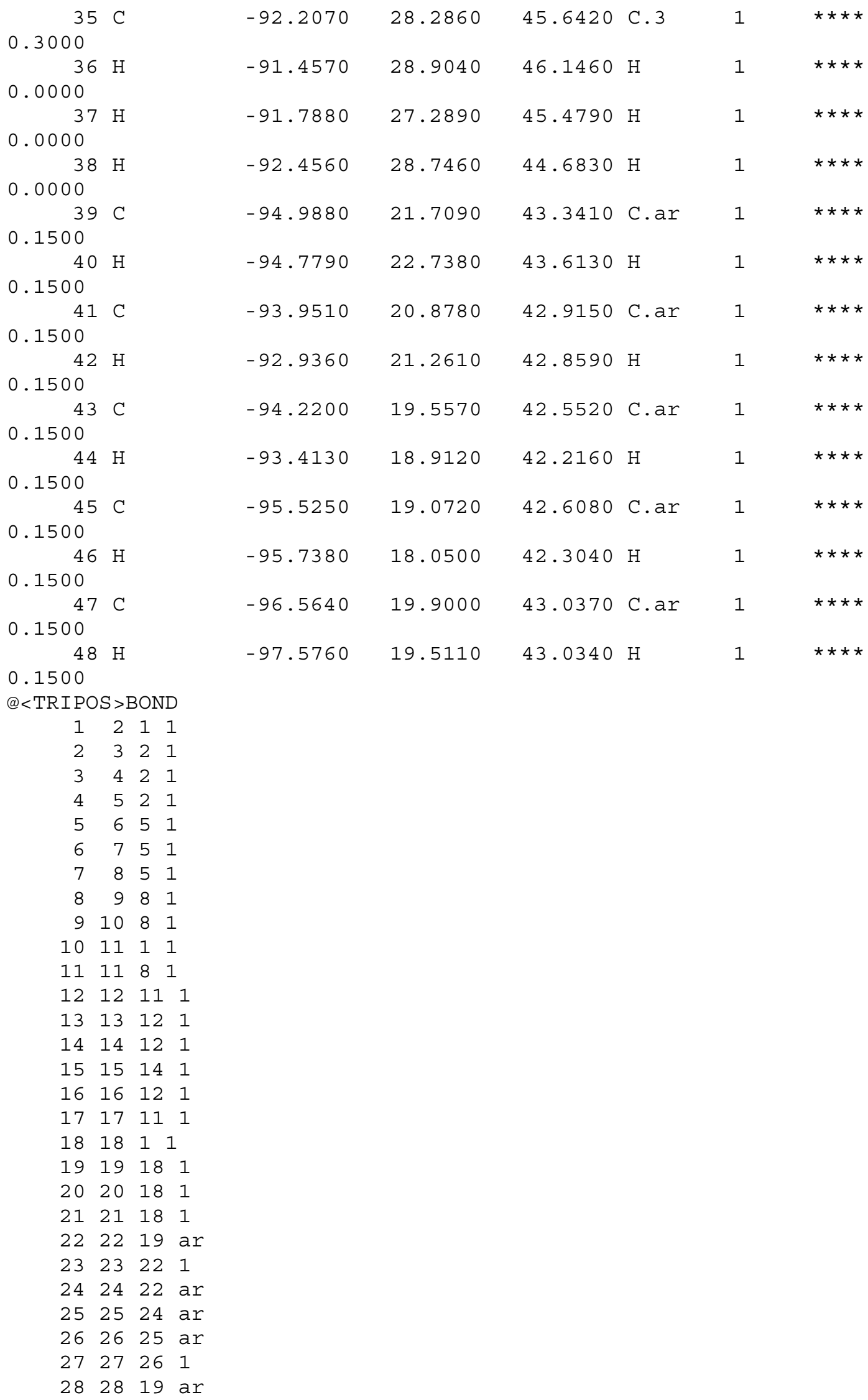




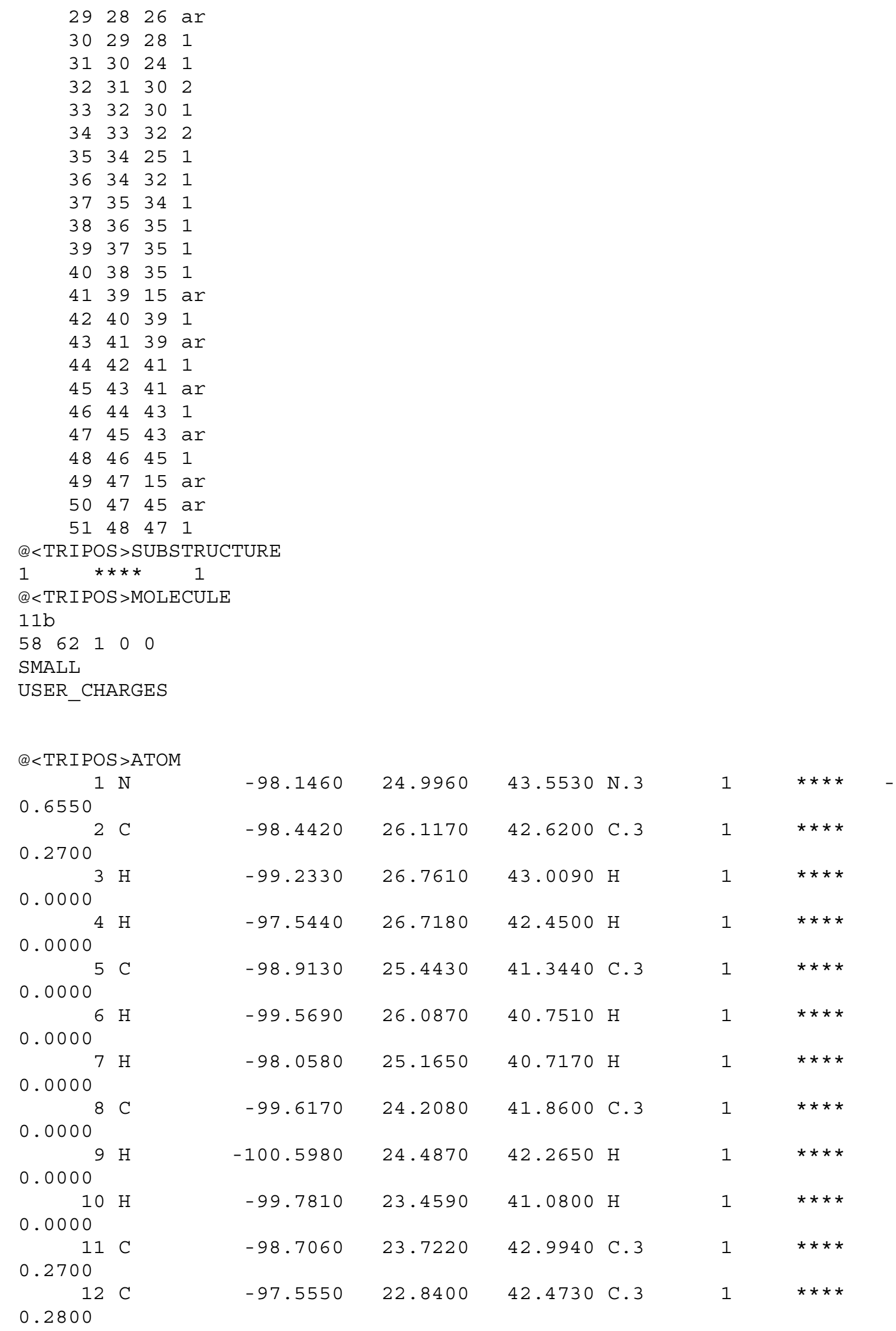




\begin{tabular}{|c|c|c|c|c|c|c|c|}
\hline 13 & $\mathrm{H}$ & -96.9260 & 23.3620 & 41.7420 & $\mathrm{H}$ & 1 & $\star \star \star \star$ \\
\hline \multicolumn{8}{|l|}{0.0000} \\
\hline 14 & O & -96.8120 & 22.2830 & 43.5730 & 0.3 & 1 & 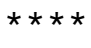 \\
\hline \multicolumn{8}{|l|}{0.3630} \\
\hline 15 & $\mathrm{C}$ & -95.7930 & 21.4170 & 43.2170 & C.ar & 1 & $\star \star \star \star *$ \\
\hline \multicolumn{8}{|c|}{0.0830} \\
\hline 16 & $\mathrm{H}$ & -97.9790 & 21.9780 & 41.9450 & $\mathrm{H}$ & 1 & 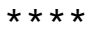 \\
\hline \multicolumn{8}{|c|}{0.0000} \\
\hline 17 & $\mathrm{H}$ & -99.2860 & 23.1740 & 43.7390 & $\mathrm{H}$ & 1 & $\star \star \star \star *$ \\
\hline \multicolumn{8}{|c|}{0.0000} \\
\hline 18 & $S$ & -98.2240 & 25.2890 & 45.2220 & S. 3 & 1 & 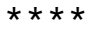 \\
\hline \multicolumn{8}{|c|}{0.5380} \\
\hline 19 & C & -96.7350 & 26.1770 & 45.5650 & C.ar & 1 & 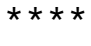 \\
\hline \multicolumn{8}{|c|}{0.0070} \\
\hline 20 & O & -99.3300 & 26.1890 & 45.4820 & 0.3 & 1 & 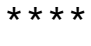 \\
\hline \multicolumn{8}{|c|}{0.2150} \\
\hline 21 & O & -98.1490 & 24.0100 & 45.8990 & 0.3 & 1 & $\star \star \star \star *$ \\
\hline \multicolumn{8}{|c|}{0.2150} \\
\hline \multicolumn{8}{|c|}{0.1500} \\
\hline \multicolumn{5}{|c|}{0.1500} & $\mathrm{H}$ & & $\star \star \star \star *$ \\
\hline $0.0860^{24}$ & \multicolumn{6}{|c|}{0.0860} & $\star \star \star \star *$ \\
\hline \multicolumn{8}{|c|}{0.1170} \\
\hline $0.1500^{26}$ & \multicolumn{6}{|c|}{0.1500} & 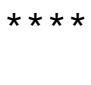 \\
\hline \multicolumn{8}{|c|}{0.1500} \\
\hline \multicolumn{8}{|c|}{0.1500} \\
\hline \multicolumn{8}{|c|}{0.1500} \\
\hline \multicolumn{8}{|c|}{0.4840} \\
\hline \multicolumn{8}{|c|}{0.5700} \\
\hline $0.6300^{32}$ & \multicolumn{6}{|c|}{0.6300} & $\star \star \star \star$ \\
\hline $0.5700^{33}$ & \multicolumn{6}{|c|}{0.5700} & $\star \star \star \star$ \\
\hline \multicolumn{8}{|c|}{0.4770} \\
\hline \multicolumn{8}{|c|}{0.4440} \\
\hline $0.0000^{36}$ & $\mathrm{H}$ & -91.6430 & 27.5020 & 45.5970 & $\mathrm{H}$ & 1 & $\star \star \star *$ \\
\hline $\begin{array}{r}37 \\
0.0000^{3}\end{array}$ & $\mathrm{H}$ & -92.2660 & 28.9900 & 44.9090 & $\mathrm{H}$ & 1 & $\star \star \star *$ \\
\hline $\begin{array}{r}38 \\
0.1440^{2}\end{array}$ & $\mathrm{C}$ & -91.0190 & 29.2280 & 46.6630 & C.ar & 1 & $\star \star \star \star *$ \\
\hline 39 & $\mathrm{C}$ & -94.5060 & 21.9550 & 43.2080 & C.ar & 1 & $\star * \star *$ \\
\hline 0.1500 & & & & & & & \\
\hline $\begin{array}{r}40 \\
0.1500^{2}\end{array}$ & $\mathrm{H}$ & -94.3490 & 22.9890 & 43.4970 & $\mathrm{H}$ & 1 & $\star \star \star \star *$ \\
\hline
\end{tabular}




\begin{tabular}{|c|c|c|c|c|c|c|c|c|c|}
\hline \multirow{2}{*}{$\begin{array}{r}41 \\
0.1500^{1}\end{array}$} & \multicolumn{2}{|l|}{$\mathrm{C}$} & -93.4210 & 21.1630 & 42.8330 & C.ar & 1 & $\star \star \star \star$ & - \\
\hline & \\
\hline 42 & \multicolumn{2}{|c|}{$\mathrm{H}$} & -92.4180 & 21.5800 & 42.8340 & $\mathrm{H}$ & 1 & $\star \star \star \star$ & \\
\hline \multicolumn{9}{|c|}{0.1500} & \\
\hline 43 & \multicolumn{2}{|c|}{$C$} & -93.6250 & 19.8370 & 42.4500 & C.ar & 1 & $\star \star \star \star$ & - \\
\hline \multicolumn{9}{|c|}{0.1500} & \\
\hline 44 & \multicolumn{2}{|c|}{$\mathrm{H}$} & -92.7800 & 19.2210 & 42.1530 & $\mathrm{H}$ & 1 & 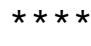 & \\
\hline \multicolumn{9}{|c|}{0.1500} & \\
\hline 45 & \multicolumn{2}{|c|}{ C } & -94.9150 & 19.3060 & 42.4320 & C.ar & 1 & 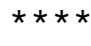 & - \\
\hline \multicolumn{9}{|c|}{0.1500} & \\
\hline 46 & \multicolumn{2}{|c|}{$\mathrm{H}$} & -95.0760 & 18.2810 & 42.1110 & $\mathrm{H}$ & 1 & 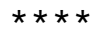 & \\
\hline \multicolumn{9}{|c|}{0.1500} & \\
\hline 47 & \multicolumn{2}{|c|}{$C$} & -96.0020 & 20.0950 & 42.8070 & C.ar & 1 & $\star \star \star \star$ & - \\
\hline \multicolumn{9}{|c|}{0.1500} & \\
\hline 48 & \multicolumn{2}{|c|}{$\mathrm{H}$} & -97.0020 & 19.6770 & 42.7510 & $\mathrm{H}$ & 1 & $\star \star \star \star$ & \\
\hline 0.1500 & & & & & & & & & \\
\hline 49 & $\mathrm{C}$ & & -90.2870 & 28.5690 & 47.6620 & C.ar & 1 & $\star \star \star \star \star$ & - \\
\hline 0.1500 & & & & & & & & & \\
\hline 50 & $\mathrm{H}$ & & -90.4410 & 27.5100 & 47.8500 & $\mathrm{H}$ & 1 & $\star \star \star \star \star$ & \\
\hline 0.1500 & & & & & & & & & \\
\hline 51 & $\mathrm{C}$ & & -89.3450 & 29.2600 & 48.4280 & C.ar & 1 & $\star \star \star \star$ & - \\
\hline 0.1500 & & & & & & & & & \\
\hline 52 & $\mathrm{H}$ & & -88.7840 & 28.7400 & 49.1980 & $\mathrm{H}$ & 1 & $\star \star \star \star \star$ & \\
\hline 0.1500 & & & & & & & & & \\
\hline 53 & $\mathrm{C}$ & & -89.1290 & 30.6170 & 48.2070 & C.ar & 1 & $\star \star \star \star$ & - \\
\hline 0.1500 & & & & & & & & & \\
\hline 54 & $\mathrm{H}$ & & -88.3960 & 31.1540 & 48.8020 & $\mathrm{H}$ & 1 & $\star \star \star \star \star$ & \\
\hline 0.1500 & & & & & & & & & \\
\hline 55 & $\mathrm{C}$ & & -89.8560 & 31.2890 & 47.2280 & C.ar & 1 & $\star \star \star \star$ & - \\
\hline 0.1500 & & & & & & & & & \\
\hline 56 & $\mathrm{H}$ & & -89.6910 & 32.3490 & 47.0580 & $\mathrm{H}$ & 1 & $\star \star \star \star$ & \\
\hline 0.1500 & & & & & & & & & \\
\hline 57 & $\mathrm{C}$ & & -90.7970 & 30.6000 & 46.4630 & C.ar & 1 & 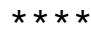 & - \\
\hline 0.1500 & & & & & & & & & \\
\hline 58 & $\mathrm{H}$ & & -91.3570 & 31.1400 & 45.7020 & $\mathrm{H}$ & 1 & $\star \star \star \star *$ & \\
\hline 0.1500 & & & & & & & & & \\
\hline @<TRIPOS & $5>B C$ & $\mathrm{ND}$ & & & & & & & \\
\hline 1 & 2 & 11 & & & & & & & \\
\hline 2 & 3 & 21 & & & & & & & \\
\hline 3 & 4 & 21 & & & & & & & \\
\hline 4 & 5 & 21 & & & & & & & \\
\hline 5 & 6 & 51 & & & & & & & \\
\hline 6 & 7 & 51 & & & & & & & \\
\hline 7 & 8 & 51 & & & & & & & \\
\hline 8 & 9 & 81 & & & & & & & \\
\hline 9 & 10 & 81 & & & & & & & \\
\hline 10 & 11 & 11 & & & & & & & \\
\hline 11 & 11 & 81 & & & & & & & \\
\hline 12 & 12 & 111 & & & & & & & \\
\hline 13 & 13 & 121 & & & & & & & \\
\hline 14 & 14 & 121 & & & & & & & \\
\hline 15 & 15 & 141 & & & & & & & \\
\hline 16 & 16 & 121 & & & & & & & \\
\hline 17 & 17 & 111 & & & & & & & \\
\hline 18 & 18 & 11 & & & & & & & \\
\hline 19 & 19 & 181 & & & & & & & \\
\hline 20 & 20 & 181 & & & & & & & \\
\hline
\end{tabular}




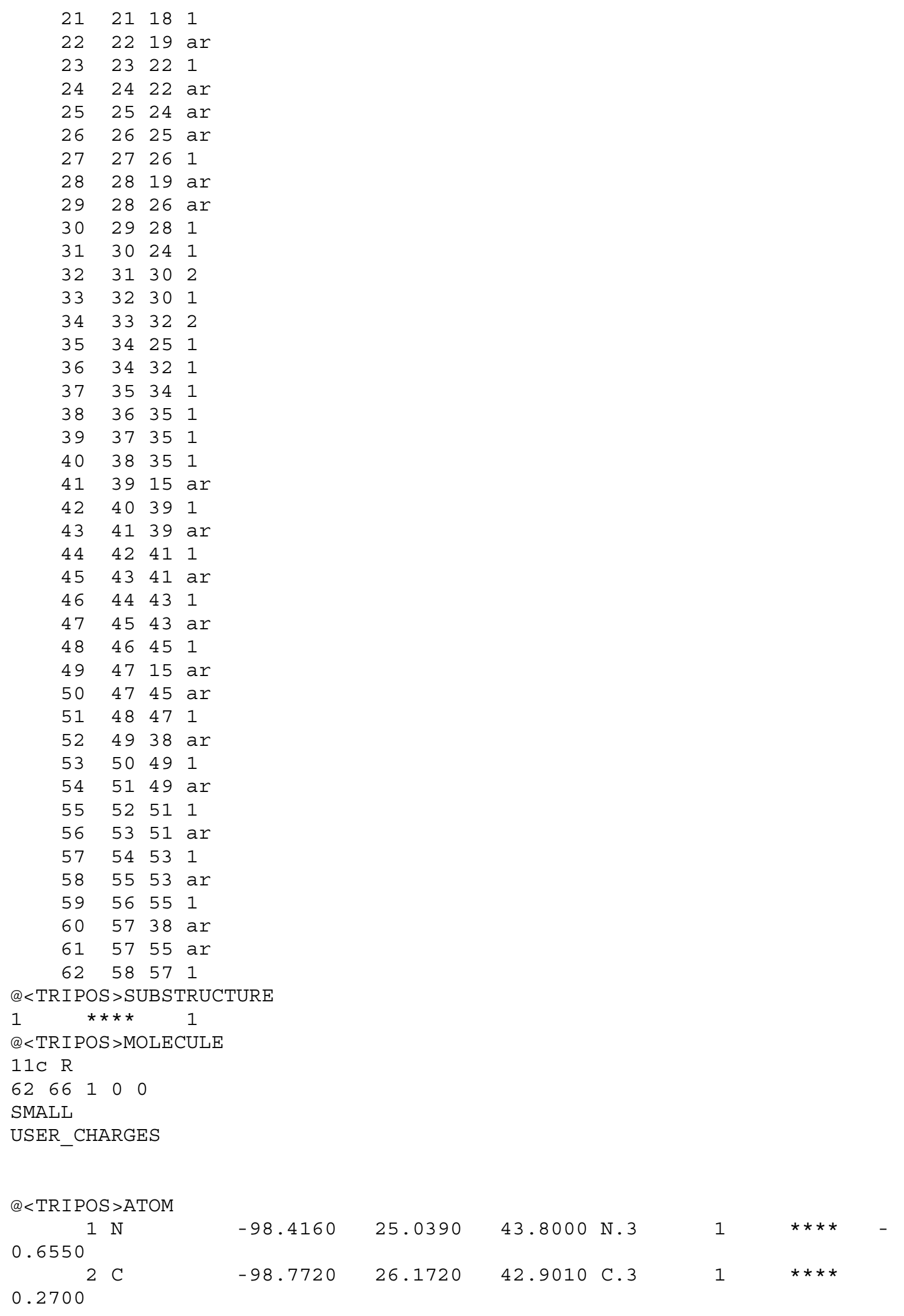




\begin{tabular}{|c|c|c|c|c|c|c|c|}
\hline 3 & $\mathrm{H}$ & -99.5440 & 26.8040 & 43.3440 & $\mathrm{H}$ & 1 & $\star \star \star \star$ \\
\hline 0.0000 & & & & & & & \\
\hline 4 & $\mathrm{H}$ & -97.8910 & 26.7780 & 42.6850 & $\mathrm{H}$ & 1 & $\star \star \star \star \star$ \\
\hline 0.0000 & & & & & & & \\
\hline 5 & $\mathrm{C}$ & -99.3140 & 25.5080 & 41.6460 & C. 3 & 1 & $\star \star \star \star *$ \\
\hline 0.0000 & & & & & & & \\
\hline 6 & $\mathrm{H}$ & -100.0000 & 26.1580 & 41.0940 & $\mathrm{H}$ & 1 & $\star \star \star \star$ \\
\hline 0.0000 & & & & & & & \\
\hline 7 & $\mathrm{H}$ & -98.4940 & 25.2310 & 40.9720 & $\mathrm{H}$ & 1 & $\star \star \star \star *$ \\
\hline 0.0000 & & & & & & & \\
\hline 8 & C & -99.9920 & 24.2710 & 42.1920 & C. 3 & 1 & 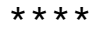 \\
\hline 0.0000 & & & & & & & \\
\hline 9 & $\mathrm{H}$ & -100.9480 & 24.5500 & 42.6540 & $\mathrm{H}$ & 1 & 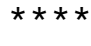 \\
\hline 0.0000 & & & & & & & \\
\hline 10 & $\mathrm{H}$ & -100.2030 & 23.5280 & 41.4180 & $\mathrm{H}$ & 1 & $\star \star \star \star$ \\
\hline 0.0000 & & & & & & & \\
\hline 11 & $\mathrm{C}$ & -99.0230 & 23.7730 & 43.2700 & C. 3 & 1 & 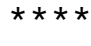 \\
\hline 0.2700 & & & & & & & \\
\hline $0.2800^{12}$ & $\mathrm{C}$ & -97.9140 & 22.8800 & 42.6820 & C. 3 & 1 & $\star \star \star \star *$ \\
\hline $0.0000^{13}$ & $\mathrm{H}$ & -97.3220 & 23.4030 & 41.9200 & $\mathrm{H}$ & 1 & 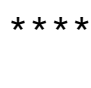 \\
\hline $0.3630^{14}$ & O & -97.1160 & 22.3130 & 43.7400 & 0.3 & 1 & $\star \star \star \star *$ \\
\hline $0.0830^{15}$ & $\mathrm{C}$ & -96.0570 & 21.5370 & 43.3100 & C.ar & 1 & $\star \star \star \star \star$ \\
\hline $0.0000^{16}$ & $\mathrm{H}$ & -98.3740 & 22.0240 & 42.1750 & $\mathrm{H}$ & 1 & 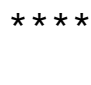 \\
\hline $0.0000^{17}$ & $\mathrm{H}$ & -99.5650 & 23.2300 & 44.0490 & $\mathrm{H}$ & 1 & $\star \star \star \star \star$ \\
\hline $0.5380^{18}$ & $S$ & -98.3760 & 25.3190 & 45.4720 & S. 3 & 1 & $\star \star \star \star *$ \\
\hline $0.0070^{19}$ & C & -96.8590 & 26.1930 & 45.7240 & C.ar & 1 & $\star \star \star \star$ \\
\hline $\begin{array}{r}20 \\
0.2150\end{array}$ & O & -99.4530 & 26.2250 & 45.8170 & 0.3 & 1 & $\star \star \star \star *$ \\
\hline $0.2150^{21}$ & O & -98.2640 & 24.0370 & 46.1360 & 0.3 & 1 & $\star \star \star \star$ \\
\hline $0.1500^{22}$ & $\mathrm{C}$ & -96.6800 & 26.9420 & 46.8900 & C.ar & 1 & 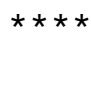 \\
\hline $0.1500^{23}$ & $\mathrm{H}$ & -97.4430 & 26.9970 & 47.6630 & $\mathrm{H}$ & 1 & 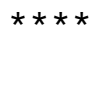 \\
\hline $\begin{array}{r}24 \\
0.0860\end{array}$ & $\mathrm{C}$ & -95.4710 & 27.6160 & 47.0660 & C.ar & 1 & $\star \star \star \star *$ \\
\hline $\begin{array}{r}25 \\
0.1170\end{array}$ & $\mathrm{C}$ & -94.4860 & 27.5670 & 46.0800 & C.ar & 1 & $\star \star \star \star *$ \\
\hline $0.1500^{26}$ & $\mathrm{C}$ & -94.6690 & 26.8620 & 44.9040 & C.ar & 1 & $\star \star \star \star *$ \\
\hline $0.1500^{27}$ & $\mathrm{H}$ & -93.9100 & 26.8330 & 44.1310 & $\mathrm{H}$ & 1 & $\star \star \star \star *$ \\
\hline $\begin{array}{r}28 \\
0.1500^{2}\end{array}$ & $\mathrm{C}$ & -95.8750 & 26.1720 & 44.7220 & C.ar & 1 & $\star \star \star \star *$ \\
\hline $\begin{array}{r}29 \\
0.1500\end{array}$ & $\mathrm{H}$ & -96.0330 & 25.6220 & 43.7990 & $\mathrm{H}$ & 1 & 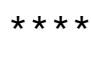 \\
\hline $0.4840^{30}$ & C & -94.9610 & 28.4380 & 48.1570 & C. 2 & 1 & $\star \star \star \star *$ \\
\hline
\end{tabular}




\begin{tabular}{|c|c|c|c|c|c|c|c|c|}
\hline 31 & O & -95.5250 & 28.7260 & 49.1970 & 0.2 & 1 & $\star \star \star \star$ & \\
\hline 0.5700 & & & & & & & & \\
\hline 32 & $\mathrm{C}$ & -93.5790 & 28.8800 & 47.7590 & C. 2 & 1 & $\star \star \star \star *$ & \\
\hline 0.6300 & & & & & & & & \\
\hline 33 & O & -92.7740 & 29.6000 & 48.3430 & 0.2 & 1 & $\star \star \star \star *$ & \\
\hline 0.5700 & & & & & & & & \\
\hline 34 & $\mathrm{~N}$ & -93.3690 & 28.3080 & 46.4910 & N.am & 1 & 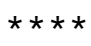 & \\
\hline 0.4770 & & & & & & & & \\
\hline 35 & $\mathrm{C}$ & -92.1540 & 28.4700 & 45.7300 & C. 3 & 1 & 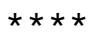 & \\
\hline 0.4440 & & & & & & & & \\
\hline 36 & $\mathrm{H}$ & -91.7800 & 27.4810 & 45.4380 & $\mathrm{H}$ & 1 & 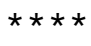 & \\
\hline 0.0000 & & & & & & & & \\
\hline 37 & $\mathrm{H}$ & -92.3920 & 28.9880 & 44.7950 & $\mathrm{H}$ & 1 & $\star \star \star \star *$ & \\
\hline 0.0000 & & & & & & & & \\
\hline 38 & $\mathrm{C}$ & -91.0540 & 29.1780 & 46.4900 & C.ar & 1 & $\star \star \star \star \star$ & \\
\hline 0.1440 & & & & & & & & \\
\hline 39 & $\mathrm{C}$ & -96.3410 & 20.2040 & 43.0180 & C.ar & 1 & $\star \star \star \star *$ & \\
\hline 0.1500 & & & & & & & & \\
\hline 40 & $\mathrm{H}$ & -97.3590 & 19.8360 & 43.1050 & $\mathrm{H}$ & 1 & $\star \star \star \star *$ & \\
\hline 0.1500 & & & & & & & & \\
\hline 41 & $\mathrm{C}$ & -95.3200 & 19.3470 & 42.6090 & C.ar & 1 & $\star \star \star \star *$ & \\
\hline 0.1500 & & & & & & & & \\
\hline 42 & $\mathrm{H}$ & -95.5430 & 18.3100 & 42.3740 & $\mathrm{H}$ & 1 & $\star \star \star \star \star$ & \\
\hline 0.1500 & & & & & & & & \\
\hline 43 & $\mathrm{C}$ & -94.0110 & 19.8170 & 42.5110 & C.ar & 1 & $\star \star \star \star *$ & \\
\hline 0.1500 & & & & & & & & \\
\hline 44 & $\mathrm{H}$ & -93.2140 & 19.1480 & 42.1980 & $\mathrm{H}$ & 1 & $\star \star \star \star *$ & \\
\hline 0.1500 & & & & & & & & \\
\hline 45 & $\mathrm{C}$ & -93.7200 & 21.1440 & 42.8300 & C.ar & 1 & 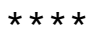 & \\
\hline 0.1500 & & & & & & & & \\
\hline 46 & $\mathrm{H}$ & -92.6970 & 21.5050 & 42.7750 & $\mathrm{H}$ & 1 & 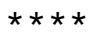 & \\
\hline 0.1500 & & & & & & & & \\
\hline 47 & $\mathrm{C}$ & -94.7390 & 22.0050 & 43.2380 & C.ar & 1 & 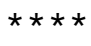 & \\
\hline 0.1500 & & & & & & & & \\
\hline 48 & $\mathrm{H}$ & -94.4940 & 23.0240 & 43.5190 & $\mathrm{H}$ & 1 & 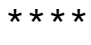 & \\
\hline 0.1500 & & & & & & & & \\
\hline 49 & $\mathrm{C}$ & -90.2400 & 28.4750 & 47.3900 & C.ar & 1 & $\star \star \star \star$ & \\
\hline 0.1500 & & & & & & & & \\
\hline 50 & $\mathrm{H}$ & -90.3770 & 27.4060 & 47.5320 & $\mathrm{H}$ & 1 & $\star \star \star \star \star$ & \\
\hline 0.1500 & & & & & & & & \\
\hline 51 & $\mathrm{C}$ & -89.2470 & 29.1310 & 48.1230 & C.ar & 1 & 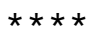 & \\
\hline 0.1500 & & & & & & & & \\
\hline 52 & $\mathrm{H}$ & -88.6490 & 28.5450 & 48.8100 & $\mathrm{H}$ & 1 & $\star \star \star \star$ & \\
\hline 0.1500 & & & & & & & & \\
\hline 53 & $\mathrm{C}$ & -89.0550 & 30.5090 & 47.9650 & C.ar & 1 & $\star \star \star \star ~$ & \\
\hline 0.0830 & & & & & & & & \\
\hline 54 & $\mathrm{C}$ & -89.8620 & 31.2150 & 47.0740 & C.ar & 1 & $\star \star \star \star$ & \\
\hline 0.1500 & & & & & & & & \\
\hline 55 & $\mathrm{H}$ & -89.7230 & 32.2860 & 46.9500 & $\mathrm{H}$ & 1 & $\star \star \star \star *$ & \\
\hline 0.1500 & & & & & & & & \\
\hline 56 & $\mathrm{C}$ & -90.8530 & 30.5590 & 46.3440 & C.ar & 1 & $\star \star \star \star \star$ & \\
\hline 0.1500 & & & & & & & & \\
\hline 57 & $\mathrm{H}$ & -91.4750 & 31.1320 & 45.6600 & $\mathrm{H}$ & 1 & $\star \star \star \star \star$ & \\
\hline 0.1500 & & & & & & & & \\
\hline $\begin{array}{r}58 \\
0.3630^{5}\end{array}$ & $\mathrm{O}$ & -88.1140 & 31.2800 & 48.6340 & 0.3 & 1 & $\star \star \star \star ~$ & \\
\hline
\end{tabular}




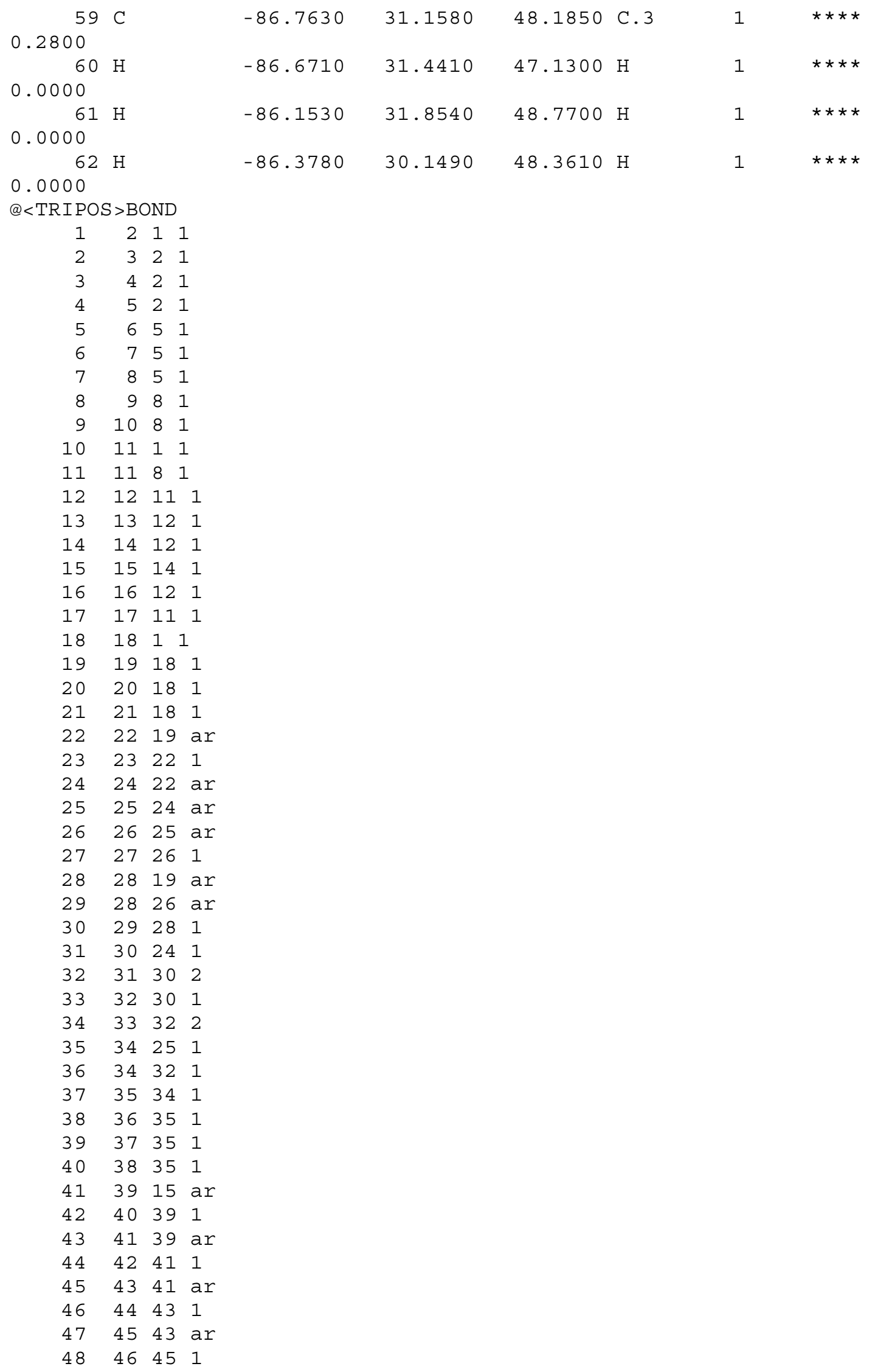




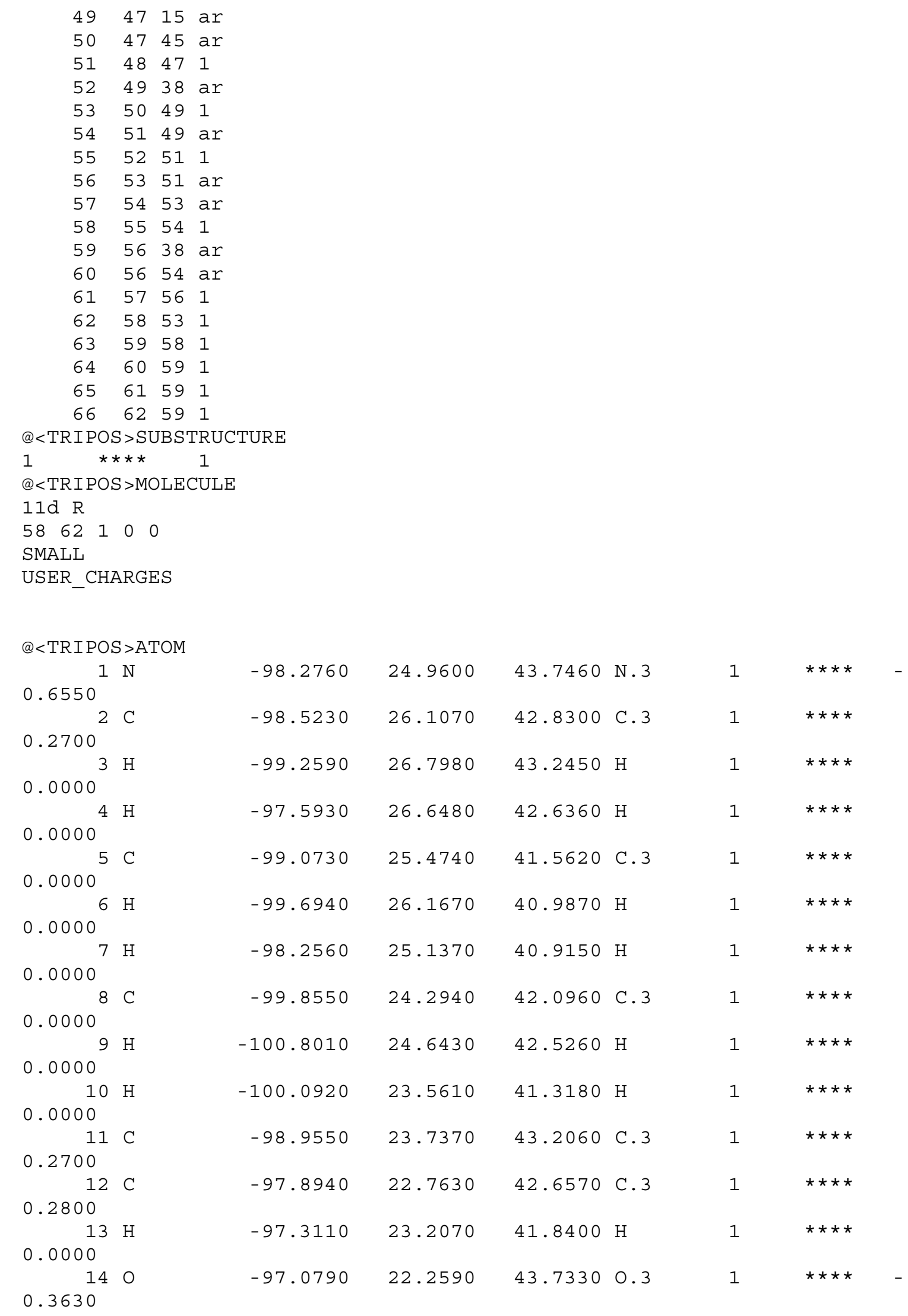




\begin{tabular}{|c|c|c|c|c|c|c|c|}
\hline 15 & $\mathrm{C}$ & -96.1300 & 21.3330 & 43.3410 & C.ar & 1 & $\star \star \star \star$ \\
\hline \multicolumn{8}{|c|}{0.0830} \\
\hline 16 & $\mathrm{H}$ & -98.3960 & 21.8860 & 42.2350 & $\mathrm{H}$ & 1 & $\star \star \star \star$ \\
\hline \multicolumn{8}{|l|}{0.0000} \\
\hline 17 & $\mathrm{H}$ & -99.5570 & 23.2390 & 43.9690 & $\mathrm{H}$ & 1 & $\star \star \star \star *$ \\
\hline \multicolumn{8}{|c|}{0.0000} \\
\hline 18 & $S$ & -98.2680 & 25.2510 & 45.4170 & S. 3 & 1 & 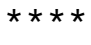 \\
\hline \multicolumn{8}{|c|}{0.5380} \\
\hline 19 & $\mathrm{C}$ & -96.7570 & 26.1290 & 45.6920 & C.ar & 1 & $\star \star \star \star *$ \\
\hline \multicolumn{8}{|c|}{0.0070} \\
\hline 20 & O & -99.3510 & 26.1590 & 45.7360 & 0.3 & 1 & 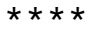 \\
\hline \multicolumn{8}{|c|}{0.2150} \\
\hline 21 & O & -98.1670 & 23.9730 & 46.0900 & 0.3 & 1 & $\star \star \star \star *$ \\
\hline \multicolumn{8}{|c|}{0.2150} \\
\hline 22 & $\mathrm{C}$ & -96.6070 & 26.8960 & 46.8520 & C.ar & 1 & $\star \star \star \star *$ \\
\hline \multicolumn{8}{|c|}{0.1500} \\
\hline $\begin{array}{r}23 \\
0.1500^{2}\end{array}$ & $\mathrm{H}$ & \multicolumn{6}{|c|}{0.1500} \\
\hline $0.0860^{24}$ & 0.0860 & -95.3990 & 27.5710 & 47.0470 & C.ar & 1 & $\star \star \star * *$ \\
\hline \multicolumn{7}{|c|}{0.1170} & $\star * \star *$ \\
\hline \multicolumn{7}{|c|}{0.1500} & $\star \star \star *$ \\
\hline \multicolumn{8}{|c|}{0.1500} \\
\hline $0.1500^{28}$ & \multicolumn{6}{|c|}{0.1500} & 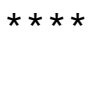 \\
\hline $0.1500^{29}$ & \multicolumn{6}{|c|}{0.1500} & $\star * \star *$ \\
\hline \multicolumn{8}{|c|}{0.4840} \\
\hline \multicolumn{8}{|c|}{0.5700} \\
\hline $0.6300^{32}$ & \multicolumn{7}{|c|}{0.6300} \\
\hline \multicolumn{8}{|c|}{0.5700} \\
\hline $\begin{array}{r}34 \\
0.4770^{3}\end{array}$ & \multicolumn{6}{|c|}{0.4770} & $\star \star \star \star$ \\
\hline \multicolumn{8}{|c|}{0.4440} \\
\hline \multicolumn{8}{|c|}{0.0000} \\
\hline \multicolumn{8}{|c|}{0.0000} \\
\hline \multicolumn{8}{|c|}{0.1440} \\
\hline $0.1500^{39}$ & $\mathrm{C}$ & -94.8040 & 21.7640 & 43.3330 & C.ar & 1 & $\star \star \star *$ \\
\hline $\begin{array}{r}40 \\
0.1500^{4}\end{array}$ & $\mathrm{H}$ & -94.5580 & 22.7710 & 43.6550 & $\mathrm{H}$ & 1 & $\star \star \star \star *$ \\
\hline $\begin{array}{r}41 \\
0.1500^{1}\end{array}$ & $\mathrm{C}$ & -93.7940 & 20.8960 & 42.9170 & C.ar & 1 & $\star \star \star \star *$ \\
\hline $0.1500^{42}$ & $\mathrm{H}$ & -92.7580 & 21.2270 & 42.9190 & $\mathrm{H}$ & 1 & $\star \star \star \star$ \\
\hline
\end{tabular}




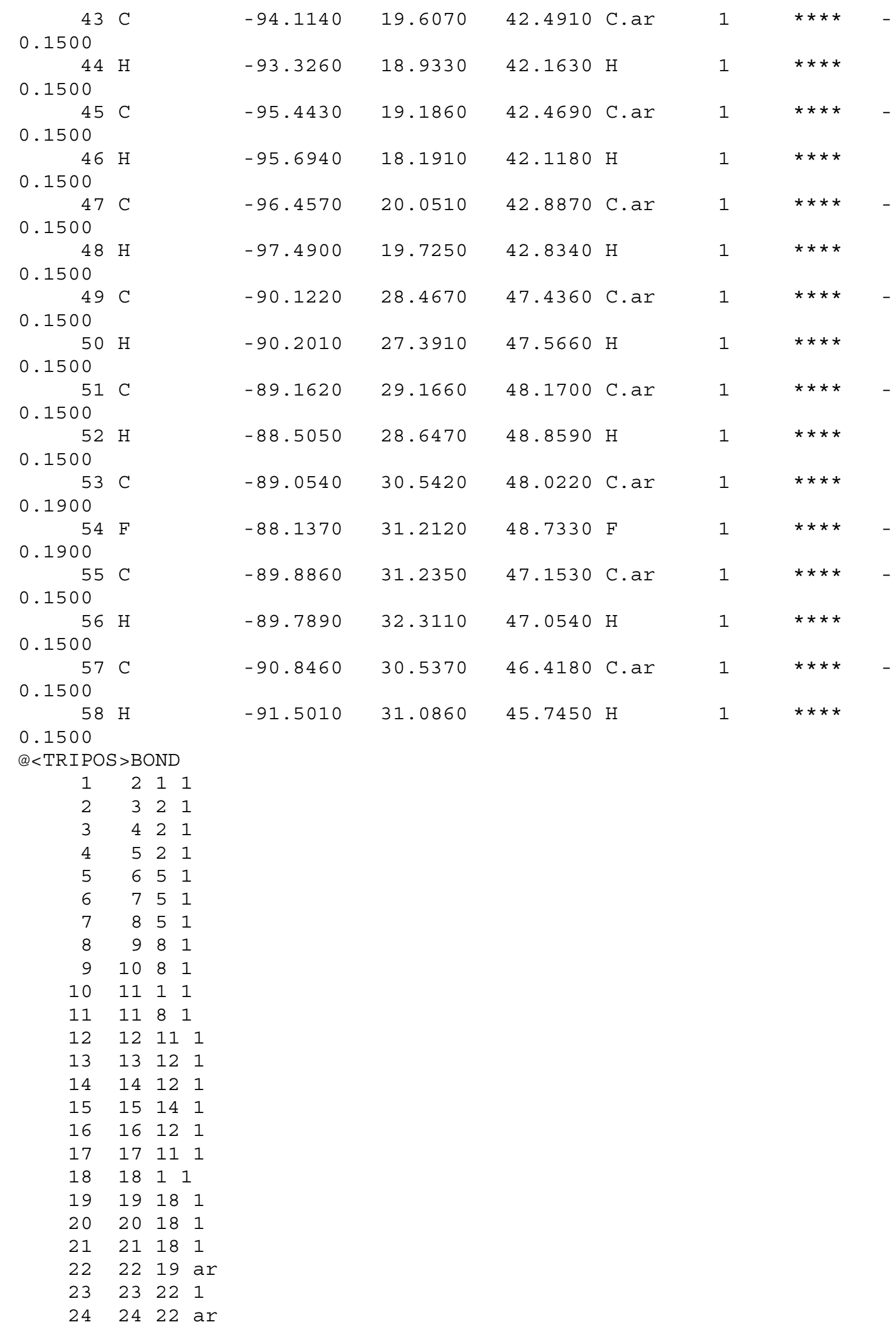




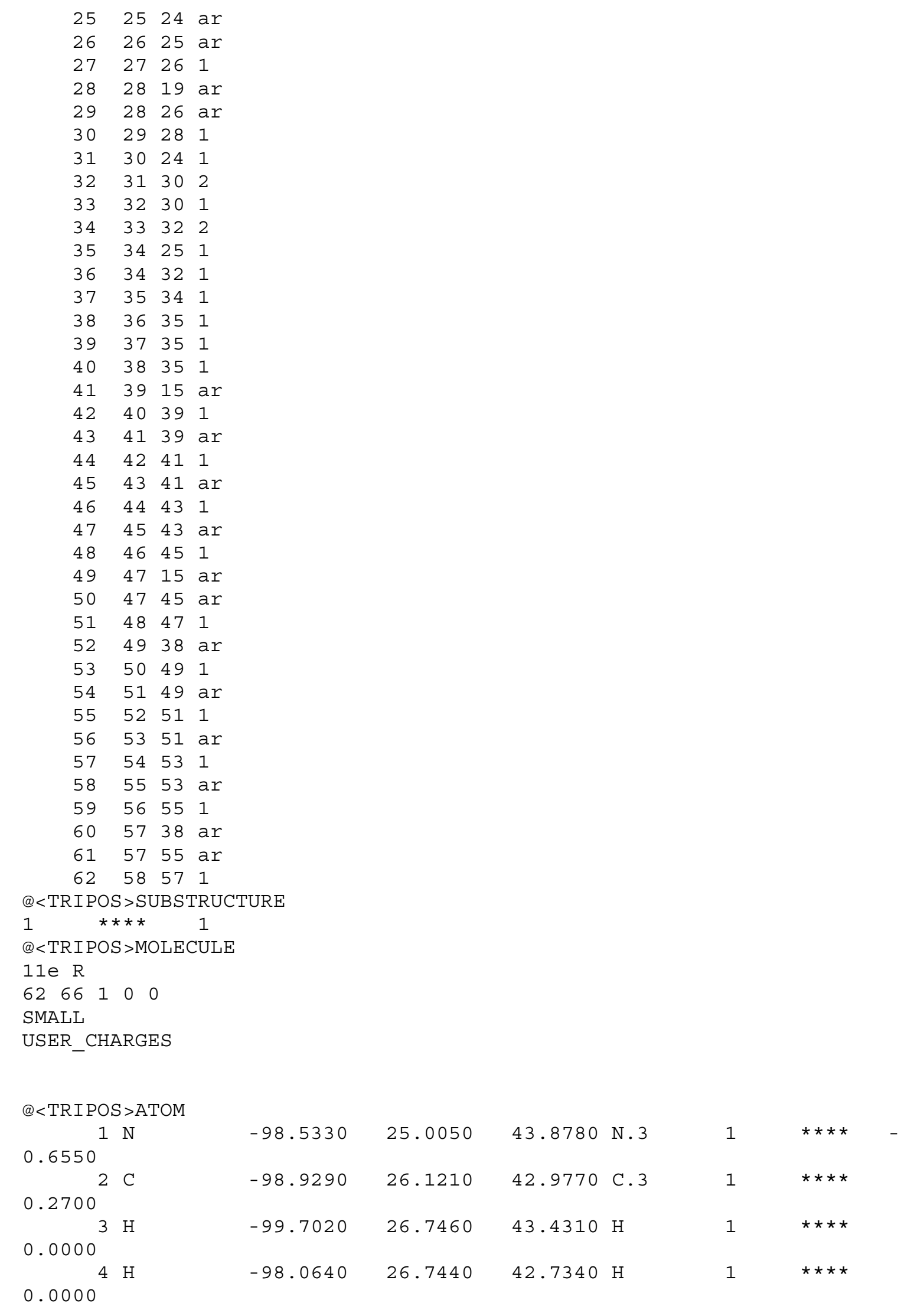




\begin{tabular}{|c|c|c|c|c|c|c|c|}
\hline \multirow{2}{*}{$0.0000^{5}$} & \multirow{2}{*}{ C } & \multirow[t]{2}{*}{-99.4890} & \multirow[t]{2}{*}{25.4370} & \multirow[t]{2}{*}{41.7410} & \multirow[t]{2}{*}{ C. 3} & \multirow[t]{2}{*}{1} & \multirow[t]{2}{*}{ 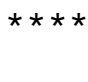 } \\
\hline & & & & & & & \\
\hline 6 & $\mathrm{H}$ & -100.1990 & 26.0690 & 41.2010 & $\mathrm{H}$ & 1 & $\star \star \star \star$ \\
\hline \multicolumn{8}{|c|}{0.0000} \\
\hline 7 & $\mathrm{H}$ & -98.6810 & 25.1720 & 41.0500 & $\mathrm{H}$ & 1 & 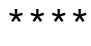 \\
\hline \multicolumn{8}{|c|}{0.0000} \\
\hline 8 & $\mathrm{C}$ & -100.1300 & 24.1930 & 42.3140 & C. 3 & 1 & $\star \star \star \star *$ \\
\hline \multicolumn{8}{|c|}{0.0000} \\
\hline 9 & $\mathrm{H}$ & -101.0800 & 24.4590 & 42.7950 & $\mathrm{H}$ & 1 & 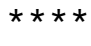 \\
\hline \multicolumn{8}{|c|}{0.0000} \\
\hline 10 & $\mathrm{H}$ & -100.3430 & 23.4390 & 41.5490 & $\mathrm{H}$ & 1 & $\star \star \star \star *$ \\
\hline \multicolumn{8}{|c|}{0.0000} \\
\hline 11 & $\mathrm{C}$ & -99.1250 & 23.7250 & 43.3720 & C. 3 & 1 & 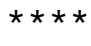 \\
\hline \multicolumn{8}{|c|}{0.2700} \\
\hline 12 & $\mathrm{C}$ & -98.0120 & 22.8480 & 42.7660 & C. 3 & 1 & 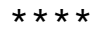 \\
\hline \multicolumn{8}{|c|}{0.2800} \\
\hline 13 & $\mathrm{H}$ & -97.3080 & 23.4360 & 42.1630 & $\mathrm{H}$ & 1 & $\star \star \star \star \star$ \\
\hline \multicolumn{8}{|c|}{0.0000} \\
\hline 14 & O & -97.3730 & 22.0740 & 43.7990 & 0.3 & 1 & 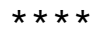 \\
\hline \multicolumn{8}{|c|}{0.3630} \\
\hline 15 & $\mathrm{C}$ & -96.3280 & 21.2850 & 43.3530 & C.ar & 1 & $\star \star \star \star \star$ \\
\hline 0.0830 & & & & & & & \\
\hline 16 & $\mathrm{H}$ & -98.4610 & 22.1150 & 42.0870 & $\mathrm{H}$ & 1 & 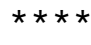 \\
\hline 0.0000 & & & & & & & \\
\hline 17 & $\mathrm{H}$ & -99.6380 & 23.1760 & 44.1660 & $\mathrm{H}$ & 1 & $\star \star \star \star \star$ \\
\hline 0.0000 & & & & & & & \\
\hline 18 & $\mathrm{~S}$ & -98.4610 & 25.3020 & 45.5470 & S. 3 & 1 & $\star \star \star \star$ \\
\hline 0.5380 & & & & & & & \\
\hline 19 & $\mathrm{C}$ & -96.9280 & 26.1550 & 45.7650 & C.ar & 1 & $\star \star \star \star \star$ \\
\hline 0.0070 & & & & & & & \\
\hline 20 & O & -99.5190 & 26.2280 & 45.8990 & 0.3 & 1 & 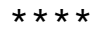 \\
\hline 0.2150 & & & & & & & \\
\hline 21 & O & -98.3580 & 24.0240 & 46.2210 & 0.3 & 1 & $\star \star \star \star \star$ \\
\hline 0.2150 & & & & & & & \\
\hline 22 & $\mathrm{C}$ & -96.7210 & 26.9170 & 46.9190 & C.ar & 1 & 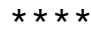 \\
\hline 0.1500 & & & & & & & \\
\hline 23 & $\mathrm{H}$ & -97.4710 & 26.9930 & 47.7020 & $\mathrm{H}$ & 1 & $\star \star \star \star \star$ \\
\hline 0.1500 & & & & & & & \\
\hline 24 & $\mathrm{C}$ & -95.4970 & 27.5760 & 47.0690 & C.ar & 1 & 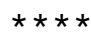 \\
\hline 0.0860 & & & & & & & \\
\hline 25 & $\mathrm{C}$ & -94.5280 & 27.4960 & 46.0690 & C.ar & 1 & 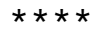 \\
\hline 0.1170 & & & & & & & \\
\hline 26 & $\mathrm{C}$ & -94.7410 & 26.7790 & 44.9050 & C.ar & 1 & 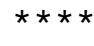 \\
\hline 0.1500 & & & & & & & \\
\hline 27 & $\mathrm{H}$ & -93.9930 & 26.7310 & 44.1220 & $\mathrm{H}$ & 1 & $\star \star \star \star *$ \\
\hline 0.1500 & & & & & & & \\
\hline 28 & $\mathrm{C}$ & -95.9580 & 26.1080 & 44.7500 & C.ar & 1 & $\star \star \star \star \star$ \\
\hline 0.1500 & & & & & & & \\
\hline 29 & $\mathrm{H}$ & -96.1390 & 25.5500 & 43.8340 & $\mathrm{H}$ & 1 & $\star \star \star \star$ \\
\hline 0.1500 & & & & & & & \\
\hline 30 & $\mathrm{C}$ & -94.9620 & 28.4070 & 48.1390 & C. 2 & 1 & $\star \star \star \star \star$ \\
\hline 0.4840 & & & & & & & \\
\hline 31 & O & -95.5100 & 28.7220 & 49.1800 & 0.2 & 1 & $\star \star \star \star \star$ \\
\hline 0.5700 & & & & & & & \\
\hline 32 & $\mathrm{C}$ & -93.5790 & 28.8220 & 47.7140 & C. 2 & 1 & $\star \star \star \star$ \\
\hline 0.6300 & & & & & & & \\
\hline
\end{tabular}




\begin{tabular}{|c|c|c|c|c|c|c|c|}
\hline 33 & 0 & -92.7570 & 29.5410 & 48.2750 & 0.2 & 1 & $\star \star \star \star$ \\
\hline \multicolumn{8}{|c|}{0.5700} \\
\hline 34 & $\mathrm{~N}$ & -93.3960 & 28.2280 & 46.4540 & N.am & 1 & $\star \star \star \star \star$ \\
\hline \multicolumn{8}{|c|}{0.4770} \\
\hline 35 & $\mathrm{C}$ & -92.1900 & 28.3620 & 45.6710 & C. 3 & 1 & $\star \star \star \star$ \\
\hline \multicolumn{8}{|c|}{0.4440} \\
\hline 36 & $\mathrm{H}$ & -91.8380 & 27.3640 & 45.3850 & $\mathrm{H}$ & 1 & $\star \star \star \star$ \\
\hline \multicolumn{8}{|c|}{0.0000} \\
\hline 37 & $\mathrm{H}$ & -92.4350 & 28.8770 & 44.7340 & $\mathrm{H}$ & 1 & $\star \star \star \star$ \\
\hline \multicolumn{8}{|c|}{0.0000} \\
\hline 38 & $\mathrm{C}$ & -91.0700 & 29.0600 & 46.4090 & C.ar & 1 & $\star \star \star \star$ \\
\hline \multicolumn{8}{|l|}{0.1440} \\
\hline 39 & $\mathrm{C}$ & -96.6600 & 20.0120 & 42.8920 & C.ar & 1 & $\star \star \star \star *$ \\
\hline \multicolumn{8}{|l|}{0.1500} \\
\hline 40 & $\mathrm{H}$ & -97.6980 & 19.6970 & 42.8660 & $\mathrm{H}$ & 1 & $\star \star \star \star$ \\
\hline \multicolumn{8}{|l|}{0.1500} \\
\hline 41 & $\mathrm{C}$ & -95.6560 & 19.1460 & 42.4560 & C.ar & 1 & $\star \star \star \star *$ \\
\hline \multicolumn{8}{|l|}{0.1500} \\
\hline 42 & $\mathrm{H}$ & -95.9120 & 18.1570 & 42.0880 & $\mathrm{H}$ & 1 & 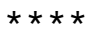 \\
\hline \multicolumn{8}{|l|}{0.1500} \\
\hline 43 & $\mathrm{C}$ & -94.3210 & 19.5490 & 42.5000 & C.ar & 1 & $\star \star \star \star *$ \\
\hline \multicolumn{8}{|l|}{0.1500} \\
\hline 44 & $\mathrm{H}$ & -93.5380 & 18.8730 & 42.1670 & $\mathrm{H}$ & 1 & $\star \star \star \star *$ \\
\hline \multicolumn{8}{|l|}{0.1500} \\
\hline 45 & $\mathrm{C}$ & -93.9860 & 20.8120 & 42.9900 & C.ar & 1 & $\star \star \star \star *$ \\
\hline \multicolumn{8}{|l|}{0.1500} \\
\hline 46 & $\mathrm{H}$ & -92.9460 & 21.1160 & 43.0450 & $\mathrm{H}$ & 1 & $\star \star \star \star *$ \\
\hline \multicolumn{8}{|l|}{0.1500} \\
\hline 47 & $\mathrm{C}$ & -94.9880 & 21.6810 & 43.4250 & C.ar & 1 & $\star \star \star \star *$ \\
\hline \multicolumn{8}{|l|}{0.1500} \\
\hline 48 & $\mathrm{H}$ & -94.7140 & 22.6470 & 43.8360 & $\mathrm{H}$ & 1 & $\star \star \star \star *$ \\
\hline \multicolumn{8}{|l|}{0.1500} \\
\hline 49 & $\mathrm{C}$ & -90.8990 & 30.4460 & 46.3020 & C.ar & 1 & 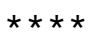 \\
\hline \multicolumn{8}{|c|}{0.1500} \\
\hline 50 & $\mathrm{H}$ & -91.5860 & 31.0420 & 45.7050 & $\mathrm{H}$ & 1 & $\star \star \star \star *$ \\
\hline \multicolumn{8}{|l|}{0.1500} \\
\hline 51 & $\mathrm{C}$ & -89.8460 & 31.0860 & 46.9580 & C.ar & 1 & $\star \star \star \star \star$ \\
\hline 0.1500 & & & & & & & \\
\hline 52 & $\mathrm{H}$ & -89.7400 & 32.1630 & 46.8570 & $\mathrm{H}$ & 1 & $\star \star \star \star *$ \\
\hline 0.1500 & & & & & & & \\
\hline 53 & $\mathrm{C}$ & -88.9330 & 30.3560 & 47.7250 & C.ar & 1 & 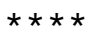 \\
\hline 0.1010 & & & & & & & \\
\hline 54 & $S$ & -87.6130 & 31.2330 & 48.5510 & S. 3 & 1 & 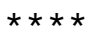 \\
\hline 0.3310 & & & & & & & \\
\hline 55 & $\mathrm{C}$ & -86.5140 & 31.5840 & 47.1490 & C. 3 & 1 & 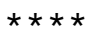 \\
\hline 0.2300 & & & & & & & \\
\hline 56 & $\mathrm{H}$ & -85.6180 & 32.1150 & 47.4840 & $\mathrm{H}$ & 1 & $\star \star \star \star *$ \\
\hline 0.0000 & & & & & & & \\
\hline 57 & $\mathrm{H}$ & -86.2050 & 30.6390 & 46.6940 & $\mathrm{H}$ & 1 & $\star \star \star \star *$ \\
\hline 0.0000 & & & & & & & \\
\hline 58 & $\mathrm{H}$ & -87.0280 & 32.1750 & 46.3860 & $\mathrm{H}$ & 1 & $\star \star \star \star *$ \\
\hline 0.0000 & & & & & & & \\
\hline 59 & $\mathrm{C}$ & -89.1060 & 28.9730 & 47.8430 & C.ar & 1 & $\star \star \star \star *$ \\
\hline 0.1500 & & & & & & & \\
\hline $\begin{array}{r}60 \\
0.1500^{6}\end{array}$ & $\mathrm{H}$ & -88.4320 & 28.3770 & 48.4510 & $\mathrm{H}$ & 1 & $\star \star \star \star *$ \\
\hline
\end{tabular}




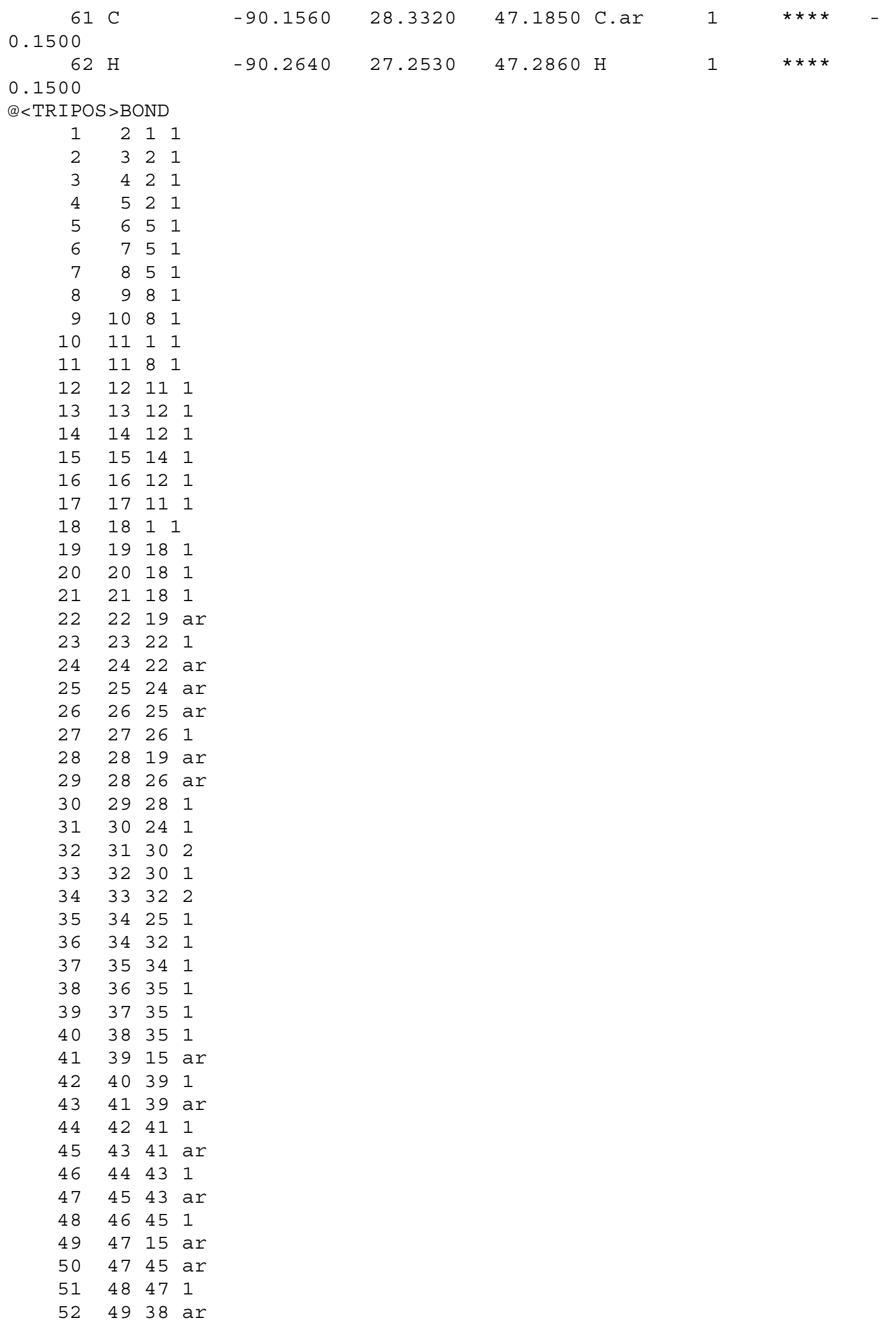




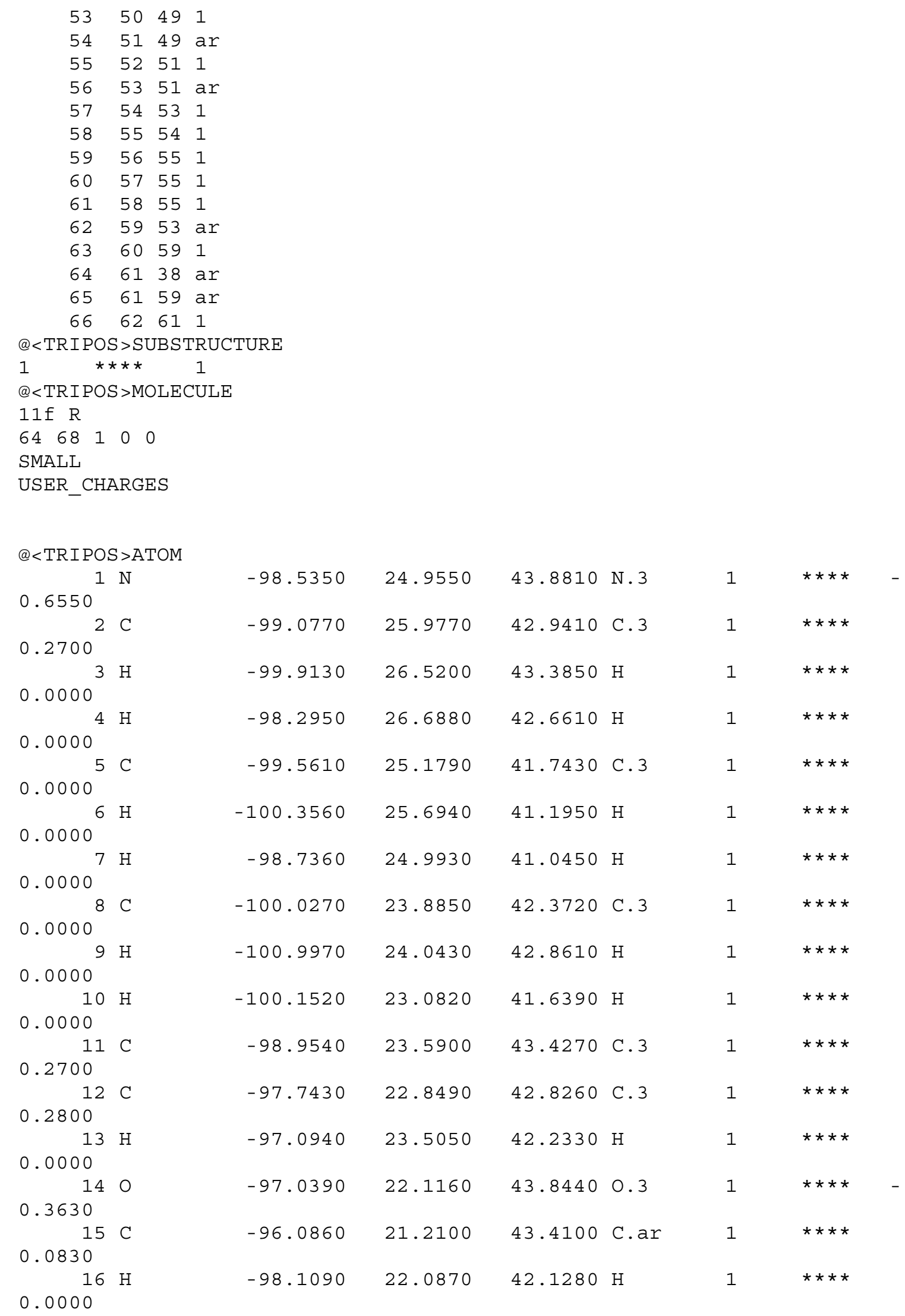




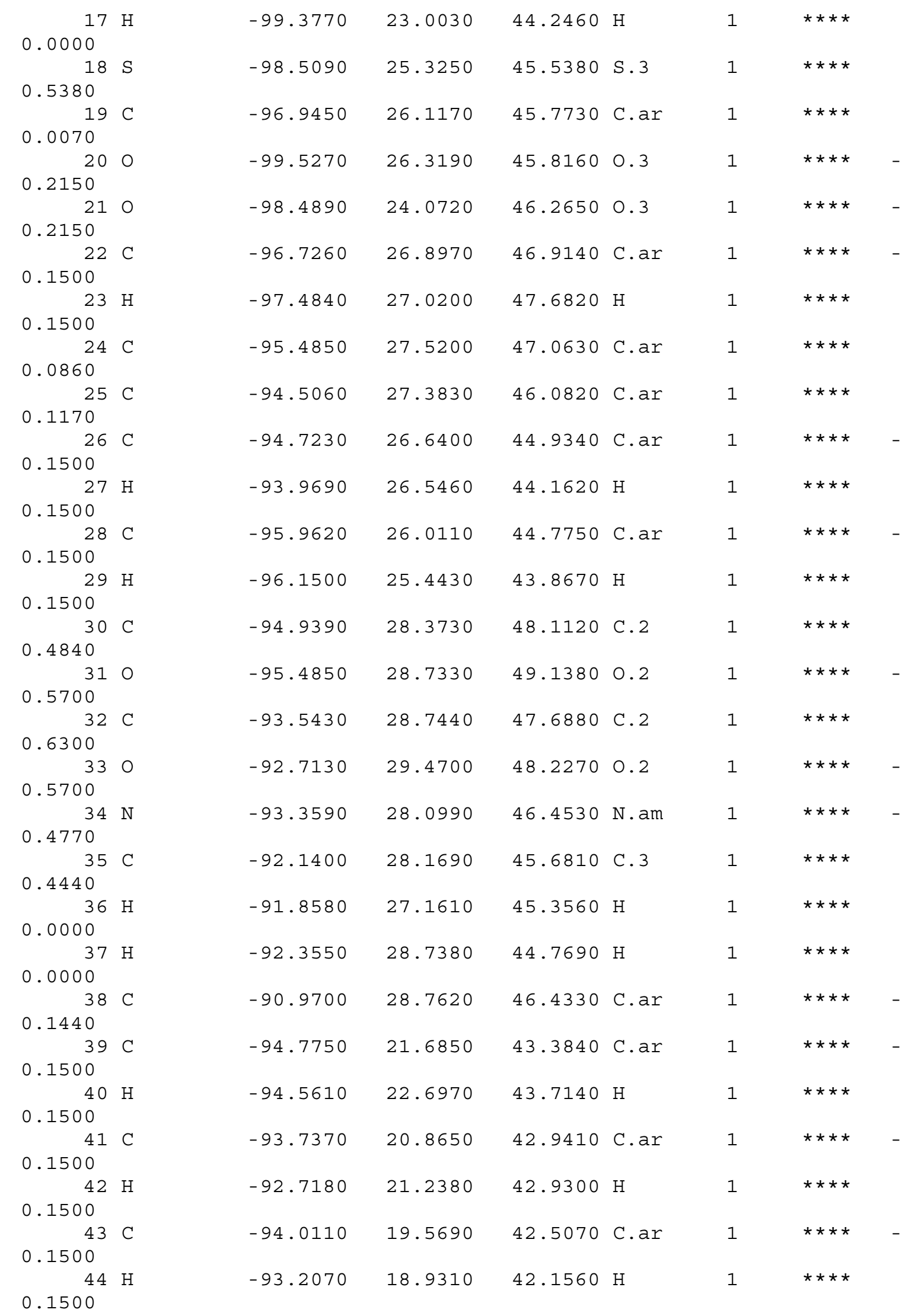




\begin{tabular}{|c|c|c|c|c|c|c|c|c|c|}
\hline \multirow{2}{*}{$\begin{array}{r}45 \\
0.1500\end{array}$} & \multicolumn{2}{|l|}{$\mathrm{C}$} & -95.3240 & 19.0970 & 42.5060 & C.ar & 1 & $\star \star \star \star$ & - \\
\hline & & & & & & & & \\
\hline 46 & \multicolumn{2}{|l|}{$\mathrm{H}$} & -95.5420 & 18.0950 & 42.1470 & $\mathrm{H}$ & 1 & $\star \star \star \star$ & \\
\hline \multicolumn{9}{|c|}{0.1500} & \\
\hline 47 & \multicolumn{2}{|l|}{$\mathrm{C}$} & -96.3640 & 19.9160 & 42.9510 & C.ar & 1 & $\star \star \star \star$ & - \\
\hline \multicolumn{9}{|c|}{0.1500} & \\
\hline 48 & \multicolumn{2}{|l|}{$\mathrm{H}$} & -97.3800 & 19.5370 & 42.9050 & $\mathrm{H}$ & 1 & 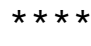 & \\
\hline \multicolumn{9}{|c|}{0.1500} & \\
\hline 49 & \multicolumn{2}{|c|}{$\mathrm{C}$} & -90.7270 & 30.1450 & 46.3830 & C.ar & 1 & 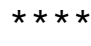 & - \\
\hline \multicolumn{9}{|c|}{0.1500} & \\
\hline 50 & \multicolumn{2}{|c|}{$\mathrm{H}$} & -91.3900 & 30.7990 & 45.8230 & $\mathrm{H}$ & 1 & 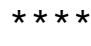 & \\
\hline \multicolumn{9}{|c|}{0.1500} & \\
\hline 51 & \multicolumn{2}{|l|}{$\mathrm{C}$} & -89.6400 & 30.7030 & 47.0540 & C.ar & 1 & $\star \star \star \star$ & - \\
\hline \multicolumn{9}{|c|}{0.1500} & \\
\hline 52 & \multicolumn{2}{|l|}{$\mathrm{H}$} & -89.4620 & 31.7730 & 47.0040 & $\mathrm{H}$ & 1 & $\star \star \star \star$ & \\
\hline 0.1500 & & & & & & & & & \\
\hline 53 & $\mathrm{C}$ & & -88.7910 & 29.8760 & 47.7900 & C.ar & 1 & $\star \star \star \star \star$ & \\
\hline 0.0830 & & & & & & & & & \\
\hline 54 & $\mathrm{C}$ & & -88.9880 & 28.4980 & 47.8230 & C.ar & 1 & $\star \star \star \star \star$ & - \\
\hline 0.1500 & & & & & & & & & \\
\hline 55 & $\mathrm{H}$ & & -88.3050 & 27.8580 & 48.3700 & $\mathrm{H}$ & 1 & $\star \star \star \star *$ & \\
\hline 0.1500 & & & & & & & & & \\
\hline 56 & $\mathrm{C}$ & & -90.0760 & 27.9430 & 47.1470 & C.ar & 1 & $\star \star \star \star \star$ & - \\
\hline 0.1500 & & & & & & & & & \\
\hline 57 & $\mathrm{H}$ & & -90.2260 & 26.8670 & 47.1760 & $\mathrm{H}$ & 1 & $\star \star \star \star \star$ & \\
\hline 0.1500 & & & & & & & & & \\
\hline 58 & $\mathrm{O}$ & & -87.6720 & 30.3990 & 48.4420 & 0.3 & 1 & $\star \star \star \star \star$ & - \\
\hline 0.2330 & & & & & & & & & \\
\hline 59 & $\mathrm{C}$ & & -86.7510 & 30.9880 & 47.5920 & C. 2 & 1 & $\star \star \star \star$ & \\
\hline 0.6590 & & & & & & & & & \\
\hline 60 & $\mathrm{C}$ & & -85.4940 & 31.2750 & 48.3600 & C. 3 & 1 & $\star \star \star \star$ & \\
\hline 0.0610 & & & & & & & & & \\
\hline 61 & $\mathrm{H}$ & & -84.6430 & 31.2830 & 47.6730 & $\mathrm{H}$ & 1 & 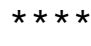 & \\
\hline 0.0000 & & & & & & & & & \\
\hline 62 & $\mathrm{H}$ & & -85.5670 & 32.2570 & 48.8360 & $\mathrm{H}$ & 1 & $\star \star \star \star *$ & \\
\hline 0.0000 & & & & & & & & & \\
\hline 63 & $\mathrm{H}$ & & -85.3300 & 30.4950 & 49.1080 & $\mathrm{H}$ & 1 & $\star \star \star \star$ & \\
\hline 0.0000 & & & & & & & & & \\
\hline 64 & $\mathrm{O}$ & & -86.8950 & 31.2500 & 46.4040 & 0.2 & 1 & 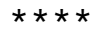 & - \\
\hline 0.5700 & & & & & & & & & \\
\hline$@<$ TRIPOS & $5>B C$ & $\mathrm{ND}$ & & & & & & & \\
\hline 1 & 2 & 11 & & & & & & & \\
\hline 2 & 3 & 21 & & & & & & & \\
\hline 3 & 4 & 21 & & & & & & & \\
\hline 4 & 5 & 21 & & & & & & & \\
\hline 5 & 6 & 51 & & & & & & & \\
\hline 6 & 7 & 51 & & & & & & & \\
\hline 7 & 8 & 51 & & & & & & & \\
\hline 8 & 9 & 81 & & & & & & & \\
\hline 9 & 10 & 81 & & & & & & & \\
\hline 10 & 11 & 11 & & & & & & & \\
\hline 11 & 11 & 81 & & & & & & & \\
\hline 12 & 12 & 111 & & & & & & & \\
\hline 13 & 13 & 121 & & & & & & & \\
\hline 14 & 14 & 121 & & & & & & & \\
\hline 15 & 15 & 141 & & & & & & & \\
\hline 16 & 16 & 121 & & & & & & & \\
\hline
\end{tabular}




\begin{tabular}{|c|c|c|c|}
\hline 17 & 17 & 11 & 1 \\
\hline 18 & 18 & 11 & \\
\hline 19 & 19 & 18 & 1 \\
\hline 20 & 20 & 18 & 1 \\
\hline 21 & 21 & 18 & 1 \\
\hline 22 & 22 & 19 & $\operatorname{ar}$ \\
\hline 23 & 23 & 22 & 1 \\
\hline 24 & 24 & 22 & $\operatorname{ar}$ \\
\hline 25 & 25 & 24 & ar \\
\hline 26 & 26 & 25 & $\operatorname{ar}$ \\
\hline 27 & 27 & 26 & 1 \\
\hline 28 & 28 & 19 & ar \\
\hline 29 & 28 & 26 & ar \\
\hline 30 & 29 & 28 & 1 \\
\hline 31 & 30 & 24 & 1 \\
\hline 32 & 31 & 30 & 2 \\
\hline 33 & 32 & 30 & 1 \\
\hline 34 & 33 & 32 & 2 \\
\hline 35 & 34 & 25 & 1 \\
\hline 36 & 34 & 32 & 1 \\
\hline 37 & 35 & 34 & 1 \\
\hline 38 & 36 & 35 & 1 \\
\hline 39 & 37 & 35 & 1 \\
\hline 40 & 38 & 35 & 1 \\
\hline 41 & 39 & 15 & ar \\
\hline 42 & 40 & 39 & 1 \\
\hline 43 & 41 & 39 & ar \\
\hline 44 & 42 & 41 & 1 \\
\hline 45 & 43 & 41 & $\operatorname{ar}$ \\
\hline 46 & 44 & 43 & 1 \\
\hline 47 & 45 & 43 & ar \\
\hline 48 & 46 & 45 & 1 \\
\hline 49 & 47 & 15 & $\operatorname{ar}$ \\
\hline 50 & 47 & 45 & ar \\
\hline 51 & 48 & 47 & 1 \\
\hline 52 & 49 & 38 & ar \\
\hline 53 & 50 & 49 & 1 \\
\hline 54 & 51 & 49 & ar \\
\hline 55 & 52 & 51 & 1 \\
\hline 56 & 53 & 51 & ar \\
\hline 57 & 54 & 53 & $\operatorname{ar}$ \\
\hline 58 & 55 & 54 & 1 \\
\hline 59 & 56 & 38 & ar \\
\hline 60 & 56 & 54 & $\operatorname{ar}$ \\
\hline 61 & 57 & 56 & 1 \\
\hline 62 & 58 & 53 & 1 \\
\hline 63 & 59 & 58 & 1 \\
\hline 64 & 60 & 59 & 1 \\
\hline 65 & 61 & 60 & 1 \\
\hline 66 & 62 & 60 & 1 \\
\hline 67 & 63 & 60 & 1 \\
\hline 68 & 64 & 59 & 2 \\
\hline \multicolumn{4}{|c|}{$@<$ TRIPOS > SUBSTRUCTURE } \\
\hline $1 \quad$ * & $\star \star \star$ & & 1 \\
\hline \multicolumn{4}{|c|}{$\begin{array}{l}@<\text { TRIPOS }>\text { MOLECULE } \\
11 \mathrm{~g}\end{array}$} \\
\hline $5963 \quad 1$ & 00 & & \\
\hline
\end{tabular}


SMALL

USER_CHARGES

$@<$ TRIPOS $>$ ATOM

0.6550

$-98.2680$

$2 \mathrm{C}$

$-98.4590$

24.9640

43.6310 N.3

0.2700

$3 \mathrm{H}$

$-99.1610$

26.1600

42.7670 C. 3

0.0000

$4 \mathrm{H}$

0.0000

$-97.5040$

26.8670

$43.2130 \mathrm{H}$

26.6630

$42.5960 \mathrm{H}$

$-99.0400$

25.6130

41.4730 C. 3

$-99.6330$

26.3590

$40.9340 \mathrm{H}$

$-98.2400$

25.2710

$40.8060 \mathrm{H}$

$-99.8700$

24.4420

41.9520 C. 3

$-100.7990$

24.8110

$42.4030 \mathrm{H}$

0.0000

$10 \mathrm{H}$

0.0000

$11 \mathrm{C}$

0.2700

$12 \mathrm{C}$

0.2800

$13 \mathrm{H}$

0.0000

140

0.3630

$15 \mathrm{C}$

0.0830

$16 \mathrm{H}$

0.0000

$17 \mathrm{H}$

0.0000

$18 \mathrm{~S}$

0.5380

$19 \mathrm{C}$

0.0070

200

0.2150

210

0.2150

$22 \mathrm{C}$

0.1500

$23 \mathrm{H}$

0.1500

$24 \mathrm{C}$

0.0860

$25 \mathrm{C}$

0.1170

$26 \mathrm{C}$

0.1500

$-100.1410$

23.7590

$41.1420 \mathrm{H}$

$-98.9930$

23.7960

43.0300 C. 3

$-97.9680$

22.8130

42.4290 C. 3

$-97.2330$

23.3180

$41.7910 \mathrm{H}$

$-97.3710$

22.0170

$43.4710 \quad 0.3$

$-96.2640$

21.2890

43.0740 C.ar

$-98.4930$

22.0990

$41.7840 \mathrm{H}$

$-99.6120$

23.2810

$43.7680 \mathrm{H}$

$-98.2590$

25.1750

$45.3140 \mathrm{~S} .3$

$-96.7660$

26.0720

45.6180 C.ar

$-99.3620$

26.0410

$45.6780 \quad 0.3$

$-98.1250$

23.8680

$45.9230 \quad 0.3$

$-96.6510$

26.8460

46.7760 C.ar

$-97.4550$

26.9220

$47.5030 \mathrm{H}$

$-95.4540$

27.5290

47.0010 C.ar

$-94.4190$

27.4620

46.0670 C.ar

$-94.5410$

26.7360

44.8950 C.ar 


\begin{tabular}{|c|c|c|c|c|c|c|c|}
\hline $\begin{array}{r}27 \\
0.1500\end{array}$ & $\mathrm{H}$ & -93.7430 & 26.6990 & 44.1630 & $\mathrm{H}$ & 1 & $\star \star \star \star$ \\
\hline $\begin{array}{r}28 \\
0.1500^{2}\end{array}$ & $\mathrm{C}$ & -95.7310 & 26.0370 & 44.6680 & C.ar & 1 & $\star \star \star \star *$ \\
\hline $\begin{array}{r}29 \\
0.1500\end{array}$ & $\mathrm{H}$ & -95.8410 & 25.4660 & 43.7490 & $\mathrm{H}$ & 1 & 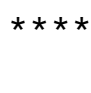 \\
\hline $0.4840^{30}$ & $\mathrm{C}$ & -95.0030 & 28.3720 & 48.0990 & C. 2 & 1 & $\star \star \star \star$ \\
\hline $0.5700^{31}$ & $\mathrm{O}$ & -95.6220 & 28.6900 & 49.0990 & 0.2 & 1 & 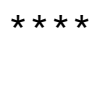 \\
\hline $0.6300^{32}$ & $\mathrm{C}$ & -93.5980 & 28.7950 & 47.7710 & C. 2 & 1 & $\star \star \star \star *$ \\
\hline $0.5700^{33}$ & O & -92.8110 & 29.4870 & 48.4130 & 0.2 & 1 & $\star \star \star \star *$ \\
\hline $\begin{array}{r}34 \\
0.4770^{3}\end{array}$ & $\mathrm{~N}$ & -93.3230 & 28.2080 & 46.5240 & N. am & 1 & $\star \star \star \star *$ \\
\hline $0.4440^{35}$ & $\mathrm{C}$ & -92.0720 & 28.3560 & 45.8220 & C. 3 & 1 & $\star \star \star \star *$ \\
\hline $0.0000^{36}$ & $\mathrm{H}$ & -91.6700 & 27.3620 & 45.5940 & $\mathrm{H}$ & 1 & $\star \star \star \star *$ \\
\hline $0.0000^{37}$ & $\mathrm{H}$ & -92.2730 & 28.8310 & 44.8540 & $\mathrm{H}$ & 1 & 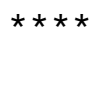 \\
\hline $\begin{array}{r}38 \\
0.1440^{3}\end{array}$ & $\mathrm{C}$ & -91.0210 & 29.1140 & 46.5990 & C.ar & 1 & $\star \star \star *$ \\
\hline $0.1500^{39}$ & C & -96.4990 & 19.9830 & 42.6460 & C.ar & 1 & 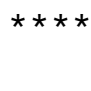 \\
\hline $\begin{array}{r}40 \\
0.1500^{4}\end{array}$ & $\mathrm{H}$ & -97.5150 & 19.5990 & 42.6080 & $\mathrm{H}$ & 1 & $\star \star \star \star *$ \\
\hline $0.1500^{41}$ & C & -95.4310 & 19.1720 & 42.2610 & C.ar & 1 & 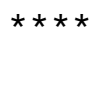 \\
\hline $0.1500^{42}$ & $\mathrm{H}$ & -95.6160 & 18.1580 & 41.9190 & $\mathrm{H}$ & 1 & 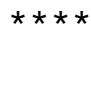 \\
\hline $0.1500^{43}$ & $\mathrm{C}$ & -94.1270 & 19.6620 & 42.3220 & C.ar & 1 & 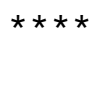 \\
\hline $0.1500^{44}$ & $\mathrm{H}$ & -93.2950 & 19.0290 & 42.0280 & $\mathrm{H}$ & 1 & $\star \star \star \star$ \\
\hline $0.1500^{45}$ & $\mathrm{C}$ & -93.8870 & 20.9590 & 42.7800 & C.ar & 1 & $\star \star \star \star$ \\
\hline $0.1500^{46}$ & $\mathrm{H}$ & -92.8700 & 21.3320 & 42.8490 & $\mathrm{H}$ & 1 & $\star \star \star \star$ \\
\hline $\begin{array}{r}47 \\
0.1500^{4}\end{array}$ & $\mathrm{C}$ & -94.9530 & 21.7730 & 43.1640 & C.ar & 1 & $\star \star \star \star$ \\
\hline $\begin{array}{r}48 \\
0.1500^{4}\end{array}$ & $\mathrm{H}$ & -94.7500 & 22.7660 & 43.5500 & $\mathrm{H}$ & 1 & $\star \star \star \star *$ \\
\hline $0.1500^{49}$ & $\mathrm{C}$ & -90.2800 & 28.4720 & 47.6060 & C.ar & 1 & $\star \star \star \star$ \\
\hline $0.1500^{50}$ & $\mathrm{H}$ & -90.4320 & 27.4150 & 47.8120 & $\mathrm{H}$ & 1 & $\star \star \star \star *$ \\
\hline $0.1500^{51}$ & $\mathrm{C}$ & -89.3410 & 29.1780 & 48.3570 & C.ar & 1 & $\star \star \star \star *$ \\
\hline $0.1500^{52}$ & $\mathrm{H}$ & -88.7730 & 28.6780 & 49.1350 & $\mathrm{H}$ & 1 & $\star \star \star \star *$ \\
\hline $0.0830^{53}$ & $\mathrm{C}$ & -89.1400 & 30.5300 & 48.1110 & C.ar & 1 & $\star \star \star \star *$ \\
\hline $0.1500^{54}$ & C & -89.8630 & 31.1940 & 47.1210 & C.ar & 1 & 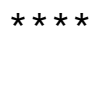 \\
\hline
\end{tabular}




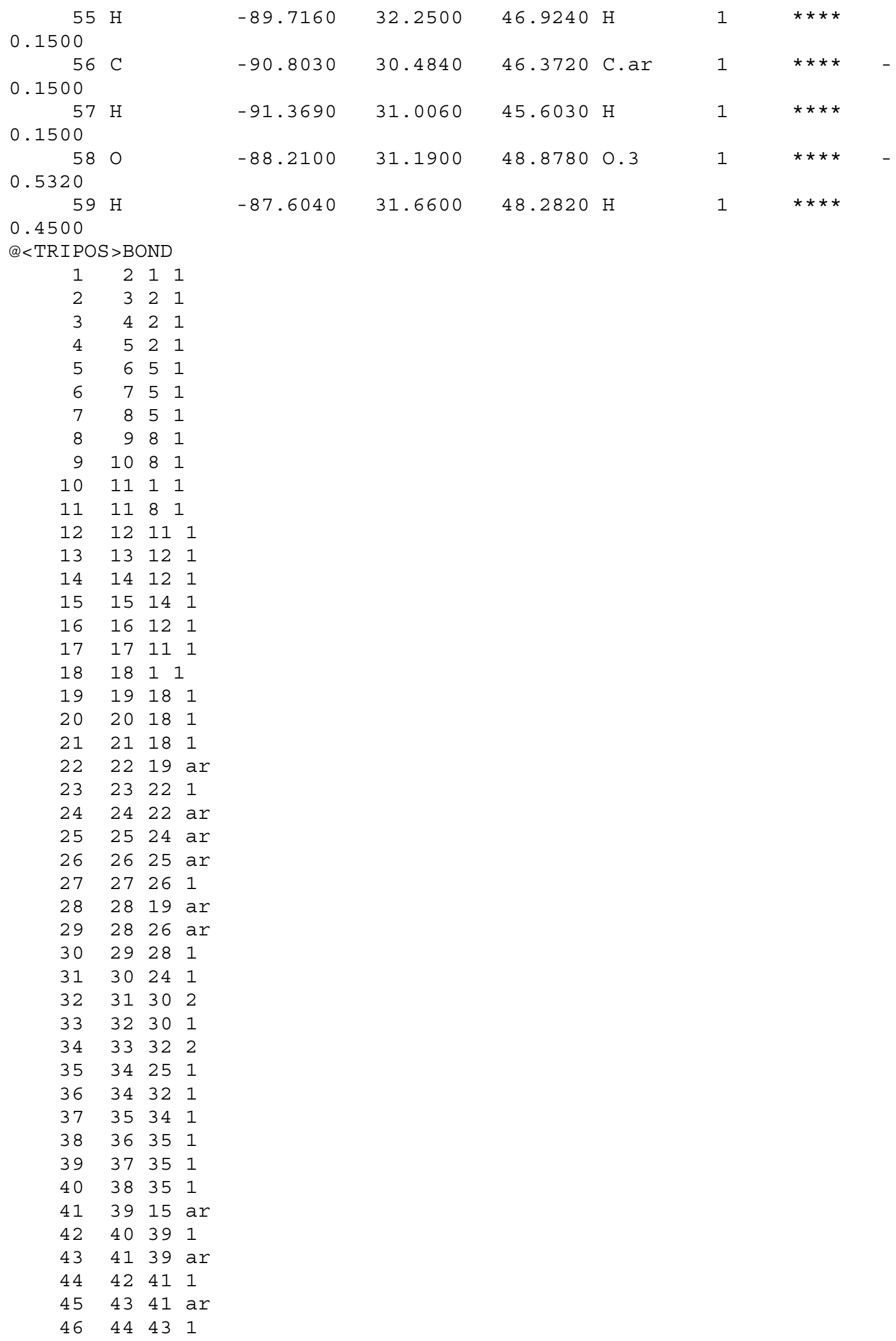




\begin{tabular}{|c|c|c|c|c|c|c|c|}
\hline & & & & & & & \\
\hline 47 & $45 \quad 43$ & $\operatorname{ar}$ & & & & & \\
\hline 48 & $46 \quad 45$ & 1 & & & & & \\
\hline 49 & $47 \quad 15$ & ar & & & & & \\
\hline 50 & $47 \quad 45$ & ar & & & & & \\
\hline 51 & $48 \quad 47$ & 1 & & & & & \\
\hline 52 & 4938 & ar & & & & & \\
\hline 53 & $50 \quad 49$ & 1 & & & & & \\
\hline 54 & 5149 & ar & & & & & \\
\hline 55 & $52 \quad 51$ & 1 & & & & & \\
\hline 56 & $53 \quad 51$ & ar & & & & & \\
\hline 57 & $54 \quad 53$ & ar & & & & & \\
\hline 58 & $55 \quad 54$ & 1 & & & & & \\
\hline 59 & 5638 & ar & & & & & \\
\hline 60 & $56 \quad 54$ & ar & & & & & \\
\hline 61 & $57 \quad 56$ & 1 & & & & & \\
\hline 62 & $58 \quad 53$ & 1 & & & & & \\
\hline 63 & 5958 & 1 & & & & & \\
\hline$@<$ TRIPOS & $S>S U B S$ & TRUCTURE & & & & & \\
\hline $1 \quad \star *$ & $\star \star \star$ & 1 & & & & & \\
\hline$@<$ TRIPOS & $\mathrm{S}>\mathrm{MOLE}$ & CULE & & & & & \\
\hline $11 \mathrm{~h} \mathrm{~S}$ & & & & & & & \\
\hline $\begin{array}{lll}57 & 61 & 1\end{array}$ & 00 & & & & & & \\
\hline SMALL & & & & & & & \\
\hline USER_CHZ & ARGES & & & & & & \\
\hline @ < TRIPOS & $\mathrm{S}>\mathrm{ATOM}$ & & & & & & \\
\hline 1 & $\mathrm{~N}$ & -98.2420 & 24.9700 & 43.7190 & N. 3 & 1 & $\star \star \star \star ~$ \\
\hline 0.6550 & & & & & & & \\
\hline 2 & $\mathrm{C}$ & -98.4700 & 26.1270 & 42.8080 & C. 3 & 1 & $\star \star \star \star$ \\
\hline 0.2700 & & & & & & & \\
\hline 3 & $\mathrm{H}$ & -99.2100 & 26.8160 & 43.2200 & $\mathrm{H}$ & 1 & $\star \star \star \star$ \\
\hline 0.0000 & & & & & & & \\
\hline 4 & $\mathrm{H}$ & -97.5350 & 26.6660 & 42.6330 & $\mathrm{H}$ & 1 & $\star \star \star \star *$ \\
\hline 0.0000 & & & & & & & \\
\hline 5 & $\mathrm{C}$ & -99.0040 & 25.5060 & 41.5290 & C. 3 & 1 & 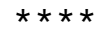 \\
\hline 0.0000 & & & & & & & \\
\hline 6 & $\mathrm{H}$ & -99.6100 & 26.2070 & 40.9480 & $\mathrm{H}$ & 1 & $\star \star \star \star *$ \\
\hline 0.0000 & & & & & & & \\
\hline 7 & $\mathrm{H}$ & -98.1800 & 25.1650 & 40.8920 & $\mathrm{H}$ & 1 & $\star \star \star \star ~$ \\
\hline 0.0000 & & & & & & & \\
\hline 8 & $\mathrm{C}$ & -99.8030 & 24.3280 & 42.0420 & C. 3 & 1 & 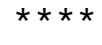 \\
\hline 0.0000 & & & & & & & \\
\hline 9 & $\mathrm{H}$ & -100.7530 & 24.6830 & 42.4580 & $\mathrm{H}$ & 1 & 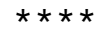 \\
\hline 0.0000 & & & & & & & \\
\hline 10 & $\mathrm{H}$ & -100.0360 & 23.6030 & 41.2560 & $\mathrm{H}$ & 1 & $\star \star \star \star$ \\
\hline 0.0000 & & & & & & & \\
\hline 11 & $\mathrm{C}$ & -98.9270 & 23.7570 & 43.1620 & C. 3 & 1 & $\star \star \star \star$ \\
\hline 0.2700 & & & & & & & \\
\hline 12 & $\mathrm{C}$ & -97.8670 & 22.7740 & 42.6250 & C. 3 & 1 & $\star \star \star \star$ \\
\hline 0.2800 & & & & & & & \\
\hline 13 & $\mathrm{H}$ & -97.1940 & 23.2530 & 41.9020 & $\mathrm{H}$ & 1 & $\star \star \star \star$ \\
\hline 0.0000 & & & & & & & \\
\hline 14 & $\mathrm{O}$ & -97.1780 & 22.1470 & 43.7230 & 0.3 & 1 & $\star \star \star \star$ \\
\hline 0.3630 & & & & & & & \\
\hline $0.0830^{15}$ & C & -96.1840 & 21.2670 & 43.3340 & C.ar & 1 & $\star \star \star \star *$ \\
\hline
\end{tabular}




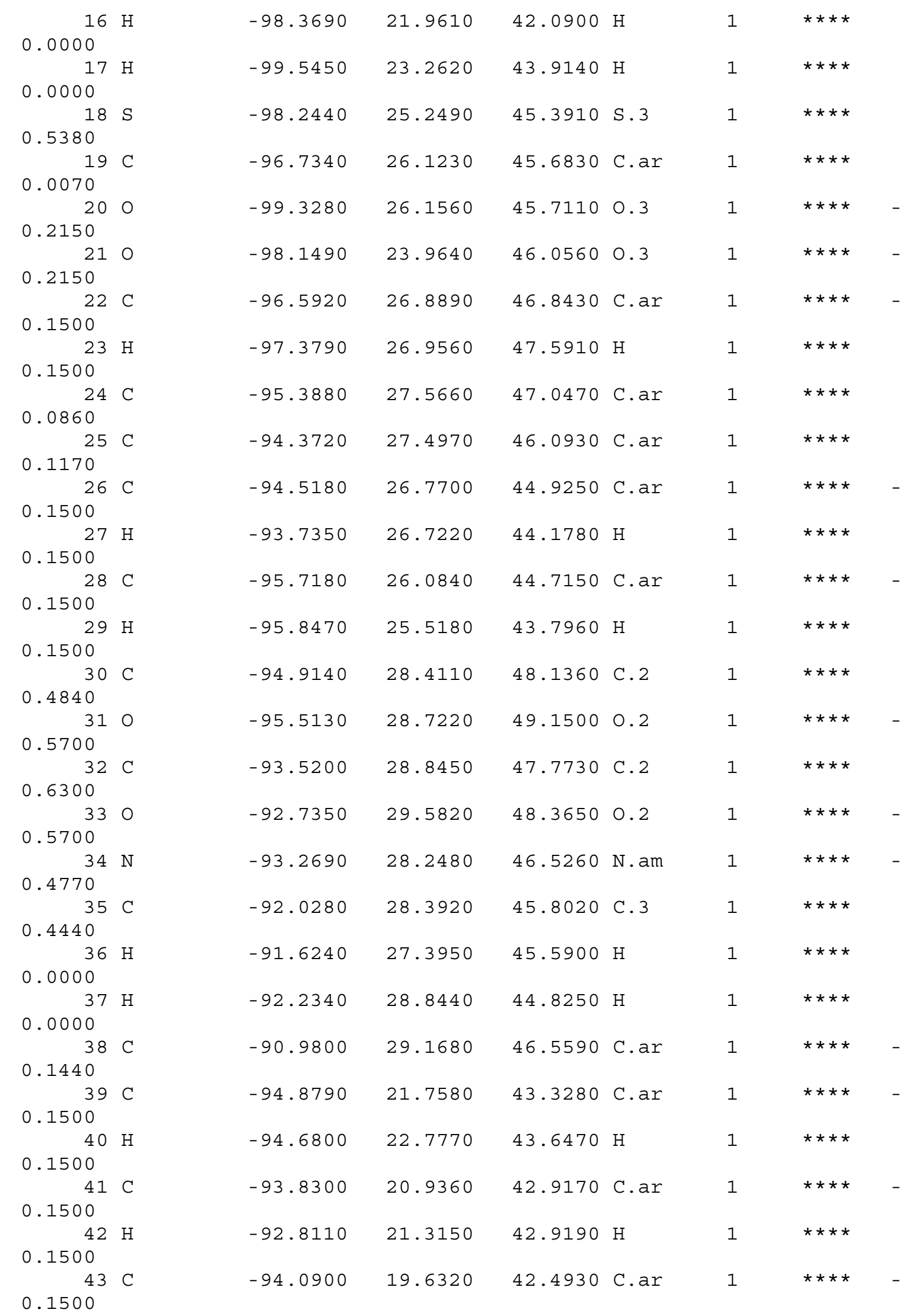




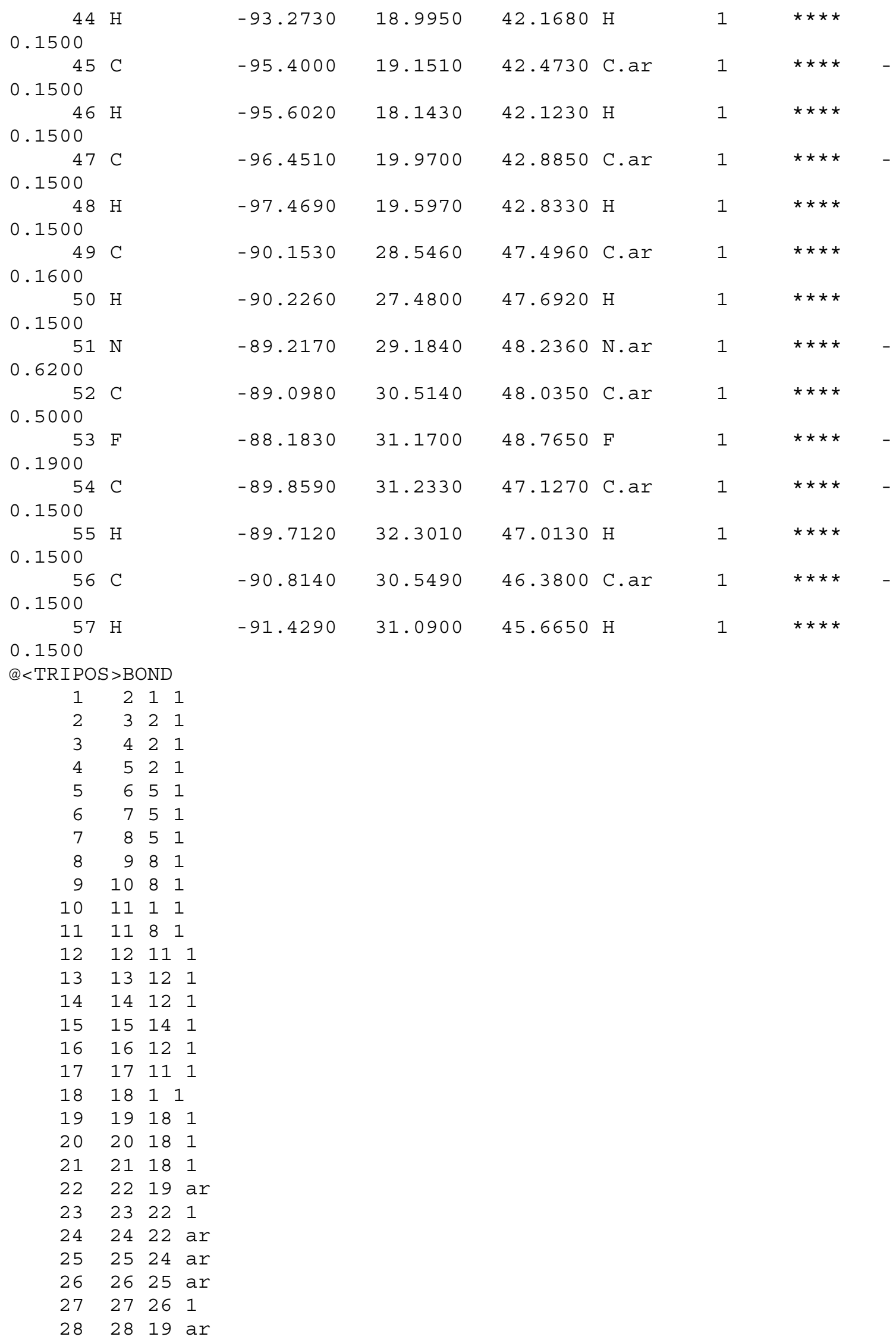




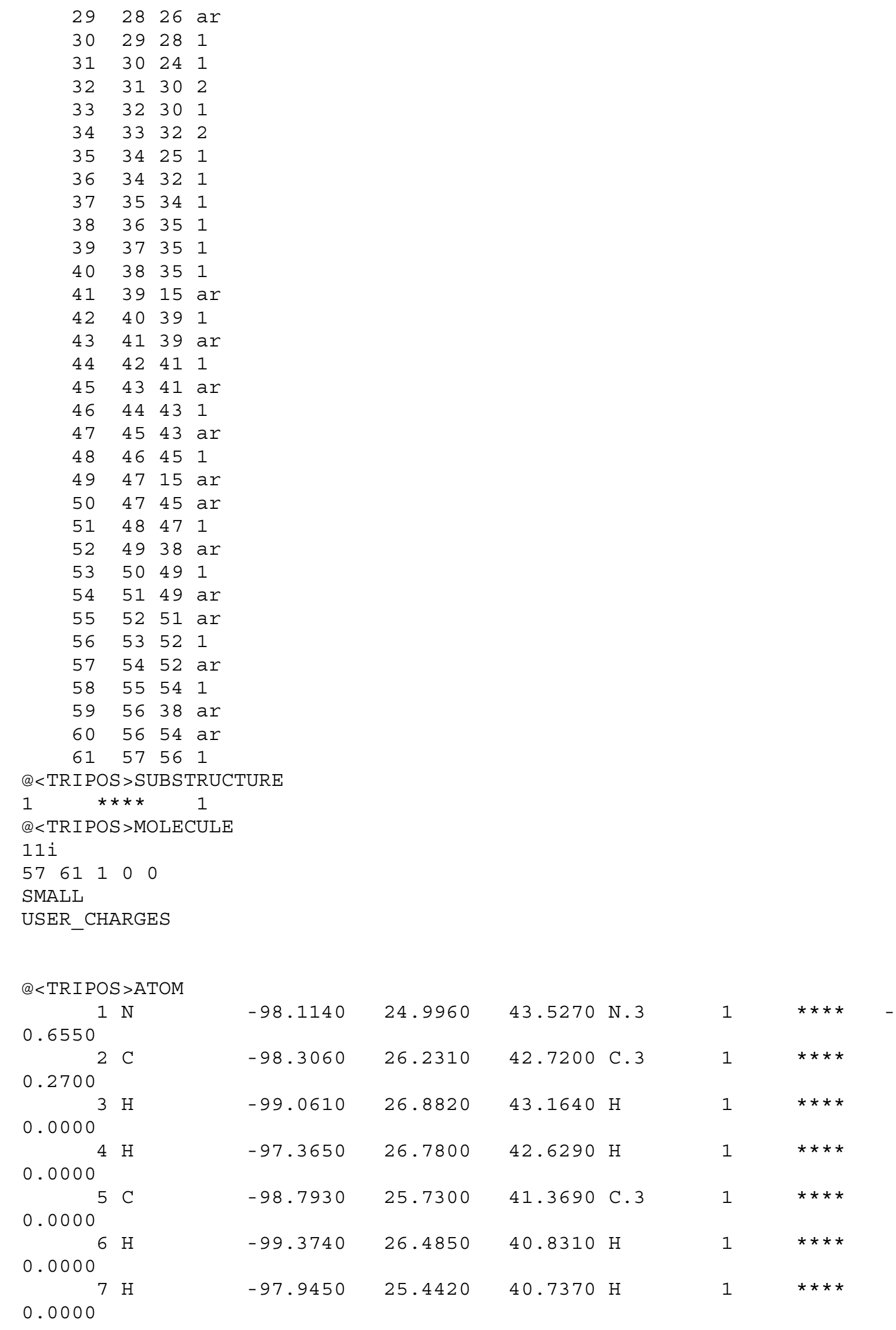




\begin{tabular}{|c|c|c|c|c|c|c|c|}
\hline 8 & $\mathrm{C}$ & -99.6140 & 24.5160 & 41.7440 & C. 3 & 1 & $\star \star \star \star \star$ \\
\hline \multicolumn{8}{|c|}{0.0000} \\
\hline 9 & $\mathrm{H}$ & -100.5780 & 24.8390 & 42.1540 & $\mathrm{H}$ & 1 & $\star \star \star \star$ \\
\hline \multicolumn{8}{|c|}{0.0000} \\
\hline 10 & $\mathrm{H}$ & -99.8170 & 23.8650 & 40.8890 & $\mathrm{H}$ & 1 & $\star \star \star \star$ \\
\hline \multicolumn{8}{|c|}{0.0000} \\
\hline 11 & $\mathrm{C}$ & -98.7820 & 23.8420 & 42.8420 & C. 3 & 1 & 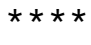 \\
\hline \multicolumn{8}{|c|}{0.2700} \\
\hline 12 & $\mathrm{C}$ & -97.7090 & 22.9020 & 42.2620 & C. 3 & 1 & 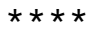 \\
\hline \multicolumn{8}{|c|}{0.2800} \\
\hline 13 & $\mathrm{H}$ & -96.9490 & 23.4740 & 41.7160 & $\mathrm{H}$ & 1 & 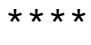 \\
\hline \multicolumn{8}{|c|}{0.0000} \\
\hline 14 & $\mathrm{O}$ & -97.0940 & 22.1750 & 43.3370 & 0.3 & 1 & $\star \star \star \star$ \\
\hline \multicolumn{8}{|c|}{0.3630} \\
\hline 15 & $\mathrm{C}$ & -96.0960 & 21.2750 & 42.9970 & C.ar & 1 & $\star \star \star \star$ \\
\hline \multirow{2}{*}{\multicolumn{8}{|c|}{$\begin{array}{r}0.0830 \\
16 \mathrm{H}\end{array}$}} \\
\hline & $\mathrm{H}$ & -98.1860 & & & & & \\
\hline \multicolumn{8}{|c|}{0.0000} \\
\hline 17 & $\mathrm{H}$ & -99.4340 & 23.2920 & 43.5260 & $\mathrm{H}$ & 1 & $\star \star \star \star$ \\
\hline \multicolumn{8}{|c|}{0.0000} \\
\hline 18 & S & -98.1240 & 25.1150 & 45.2150 & S. 3 & 1 & $\star \star \star \star ~$ \\
\hline \multicolumn{8}{|c|}{0.5380} \\
\hline 19 & C & -96.6490 & 26.0190 & 45.5740 & C.ar & 1 & $\star \star \star \star$ \\
\hline \multirow{2}{*}{\multicolumn{8}{|c|}{$\begin{array}{r}0.0070 \\
200\end{array}$}} \\
\hline & $\mathrm{O}$ & -99.2440 & & & & & \\
\hline \multicolumn{8}{|c|}{0.2150} \\
\hline 21 & $\mathrm{O}$ & -97.9690 & 23.7790 & 45.7550 & 0.3 & 1 & $\star \star \star \star$ \\
\hline \multicolumn{8}{|c|}{$\begin{array}{r}0.2150 \\
22 \mathrm{C}\end{array}$} \\
\hline \multicolumn{8}{|c|}{0.1500} \\
\hline \multicolumn{7}{|c|}{0.1500} & $\star \star \star \star$ \\
\hline \multicolumn{8}{|c|}{0.0860} \\
\hline 25 & $\mathrm{C}$ & -94.3420 & 27.4440 & 46.0910 & C.ar & 1 & $\star \star \star \star *$ \\
\hline \multicolumn{8}{|c|}{0.1170} \\
\hline 26 & $\mathrm{C}$ & -94.4090 & 26.7000 & 44.9260 & C.ar & 1 & 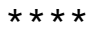 \\
\hline \multicolumn{8}{|c|}{0.1500} \\
\hline 27 & $\mathrm{H}$ & -93.5870 & 26.6670 & 44.2200 & $\mathrm{H}$ & 1 & $\star \star \star \star$ \\
\hline \multicolumn{8}{|c|}{0.1500} \\
\hline $\begin{array}{r}28 \\
0.1500^{2}\end{array}$ & $\mathrm{C}$ & -95.5800 & 25.9840 & 44.6630 & C.ar & 1 & $\star \star \star \star$ \\
\hline 29 & $\mathrm{H}$ & -95.6510 & 25.4050 & 43.7460 & $\mathrm{H}$ & 1 & $\star \star \star \star$ \\
\hline 0.1500 & & & & & & & \\
\hline 30 & $\mathrm{C}$ & -95.0030 & 28.3670 & 48.0930 & C. 2 & 1 & 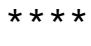 \\
\hline 0.4840 & & & & & & & \\
\hline 31 & $\mathrm{O}$ & -95.6560 & 28.6750 & 49.0740 & 0.2 & 1 & $\star \star \star \star$ \\
\hline 0.5700 & & & & & & & \\
\hline 32 & $\mathrm{C}$ & -93.6040 & 28.8320 & 47.7910 & C. 2 & 1 & $\star \star \star \star$ \\
\hline 0.6300 & & & & & & & \\
\hline 33 & $\mathrm{O}$ & -92.8480 & 29.5550 & 48.4350 & 0.2 & 1 & $\star \star \star \star \star$ \\
\hline 0.5700 & & & & & & & \\
\hline 34 & $\mathrm{~N}$ & -93.2890 & 28.2460 & 46.5530 & N. am & 1 & $\star \star \star \star$ \\
\hline 0.4770 & & & & & & & \\
\hline $0.4440^{35}$ & C & -92.0480 & 28.4630 & 45.8490 & C. 3 & 1 & $\star * * *$ \\
\hline
\end{tabular}




\begin{tabular}{|c|c|c|c|c|c|c|c|c|}
\hline \multirow{2}{*}{$0.0000^{36}$} & \multicolumn{2}{|l|}{$\mathrm{H}$} & -91.6300 & 27.4980 & 45.5400 & $\mathrm{H}$ & 1 & $\star \star \star \star$ \\
\hline & & & & & & & & \\
\hline 37 & \multicolumn{2}{|c|}{$\mathrm{H}$} & -92.2790 & 29.0190 & 44.9330 & $\mathrm{H}$ & 1 & $\star \star \star \star$ \\
\hline \multicolumn{9}{|c|}{0.0000} \\
\hline 38 & \multicolumn{2}{|l|}{ C } & -91.0070 & 29.1860 & 46.6730 & C.ar & 1 & $\star \star \star \star$ \\
\hline \multicolumn{9}{|c|}{0.1440} \\
\hline 39 & \multicolumn{2}{|l|}{ C } & -96.2850 & 19.9300 & 42.6530 & C.ar & 1 & $\star \star \star \star$ \\
\hline \multicolumn{9}{|c|}{0.1500} \\
\hline 40 & \multicolumn{2}{|l|}{$\mathrm{H}$} & -97.2690 & 19.4780 & 42.6240 & $\mathrm{H}$ & 1 & $\star \star \star \star$ \\
\hline \multicolumn{9}{|c|}{0.1500} \\
\hline 41 & \multicolumn{2}{|c|}{$\mathrm{C}$} & -95.1830 & 19.1310 & 42.3350 & C.ar & 1 & $\star \star \star \star$ \\
\hline \multicolumn{9}{|c|}{0.1500} \\
\hline 42 & \multicolumn{2}{|c|}{$\mathrm{H}$} & -95.3290 & 18.0890 & 42.0660 & $\mathrm{H}$ & 1 & $\star \star \star \star$ \\
\hline \multicolumn{9}{|c|}{0.1500} \\
\hline 43 & \multicolumn{2}{|c|}{$\mathrm{C}$} & -93.8960 & 19.6670 & 42.3610 & C.ar & 1 & $\star \star \star \star$ \\
\hline \multicolumn{9}{|c|}{0.1500} \\
\hline 44 & $\mathrm{H}$ & & -93.0410 & 19.0420 & 42.1150 & $\mathrm{H}$ & 1 & $\star \star \star \star$ \\
\hline 0.1500 & & & & & & & & \\
\hline 45 & $\mathrm{C}$ & & -93.7060 & 21.0050 & 42.7050 & C.ar & 1 & $\star \star \star \star ~$ \\
\hline 0.1500 & & & & & & & & \\
\hline 46 & $\mathrm{H}$ & & -92.7050 & 21.4250 & 42.7240 & $\mathrm{H}$ & 1 & $\star \star \star \star \star$ \\
\hline 0.1500 & & & & & & & & \\
\hline 47 & $\mathrm{C}$ & & -94.8040 & 21.8050 & 43.0200 & C.ar & 1 & $\star \star \star \star \star$ \\
\hline 0.1500 & & & & & & & & \\
\hline 48 & $\mathrm{H}$ & & -94.6560 & 22.8490 & 43.2830 & $\mathrm{H}$ & 1 & $\star \star \star \star \star$ \\
\hline 0.1500 & & & & & & & & \\
\hline 49 & $\mathrm{C}$ & & -90.7390 & 30.5400 & 46.4400 & C.ar & 1 & $\star \star \star \star$ \\
\hline 0.1500 & & & & & & & & \\
\hline 50 & $\mathrm{H}$ & & -91.2740 & 31.1000 & 45.6800 & $\mathrm{H}$ & 1 & $\star \star \star \star$ \\
\hline 0.1500 & & & & & & & & \\
\hline 51 & $\mathrm{C}$ & & -89.7730 & 31.1760 & 47.2120 & C.ar & 1 & 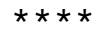 \\
\hline 0.1600 & & & & & & & & \\
\hline 52 & $\mathrm{H}$ & & -89.5410 & 32.2260 & 47.0610 & $\mathrm{H}$ & 1 & $\star \star \star \star$ \\
\hline 0.1500 & & & & & & & & \\
\hline 53 & $\mathrm{~N}$ & & -89.0640 & 30.5660 & 48.1840 & N.ar & 1 & 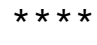 \\
\hline 0.6200 & & & & & & & & \\
\hline 54 & $\mathrm{C}$ & & -89.3250 & 29.2560 & 48.3820 & C.ar & 1 & $\star \star \star \star$ \\
\hline 0.5000 & & & & & & & & \\
\hline 55 & $F$ & & -88.6180 & 28.6240 & 49.3310 & $F$ & 1 & $\star \star \star \star$ \\
\hline 0.1900 & & & & & & & & \\
\hline 56 & $\mathrm{C}$ & & -90.2570 & 28.5280 & 47.6600 & C.ar & 1 & $\star \star \star \star$ \\
\hline 0.1500 & & & & & & & & \\
\hline 57 & $\mathrm{H}$ & & -90.3960 & 27.4740 & 47.8790 & $\mathrm{H}$ & 1 & $\star \star \star \star$ \\
\hline 0.1500 & & & & & & & & \\
\hline @<TRIPOS & $5>B C$ & ND & & & & & & \\
\hline 1 & 2 & 11 & & & & & & \\
\hline 2 & 3 & 21 & & & & & & \\
\hline 3 & 4 & 21 & & & & & & \\
\hline 4 & 5 & 21 & & & & & & \\
\hline 5 & 6 & 51 & & & & & & \\
\hline 6 & 7 & 51 & & & & & & \\
\hline 7 & 8 & 51 & & & & & & \\
\hline 8 & 9 & 81 & & & & & & \\
\hline 9 & 10 & 81 & & & & & & \\
\hline 10 & 11 & 11 & & & & & & \\
\hline 11 & 11 & 81 & & & & & & \\
\hline 12 & 12 & 111 & & & & & & \\
\hline
\end{tabular}




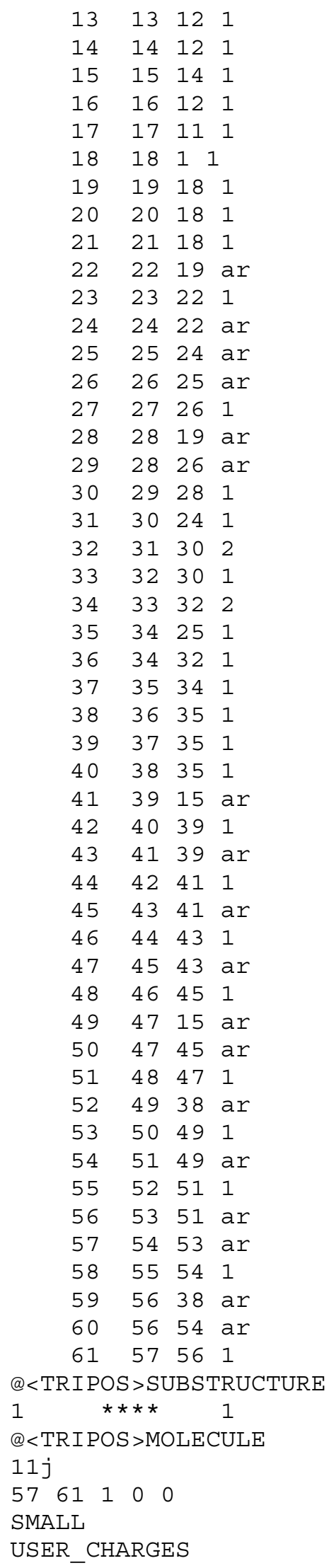




\begin{tabular}{|c|c|c|c|c|c|c|c|c|}
\hline \multicolumn{8}{|c|}{$@<$ TRIPOS >ATOM } & \multirow[b]{2}{*}{-} \\
\hline 1 & $\mathrm{~N}$ & -98.3380 & 24.9910 & 43.6340 & N. 3 & 1 & $\star \star \star \star$ & \\
\hline \multicolumn{9}{|c|}{0.6550} \\
\hline 2 & $\mathrm{C}$ & -98.5910 & 26.1650 & 42.7560 & C. 3 & 1 & $\star \star \star \star$ & \\
\hline \multicolumn{9}{|c|}{0.2700} \\
\hline 3 & $\mathrm{H}$ & -99.3320 & 26.8400 & 43.1910 & $\mathrm{H}$ & 1 & $\star \star \star \star$ & \\
\hline \multicolumn{9}{|c|}{0.0000} \\
\hline 4 & $\mathrm{H}$ & -97.6650 & 26.7190 & 42.5820 & $\mathrm{H}$ & 1 & $\star \star \star \star$ & \\
\hline \multicolumn{9}{|c|}{0.0000} \\
\hline 5 & $\mathrm{C}$ & -99.1380 & 25.5730 & 41.4670 & C. 3 & 1 & $\star \star \star \star$ & \\
\hline \multicolumn{9}{|c|}{0.0000} \\
\hline 6 & $\mathrm{H}$ & -99.7730 & 26.2780 & 40.9220 & $\mathrm{H}$ & 1 & $\star \star \star \star$ & \\
\hline \multicolumn{9}{|c|}{0.0000} \\
\hline 7 & $\mathrm{H}$ & -98.3190 & 25.2760 & 40.8030 & $\mathrm{H}$ & 1 & $\star \star \star \star$ & \\
\hline \multicolumn{9}{|c|}{0.0000} \\
\hline 8 & $\mathrm{C}$ & -99.8970 & 24.3590 & 41.9540 & C. 3 & 1 & $\star \star \star \star *$ & \\
\hline \multicolumn{9}{|c|}{0.0000} \\
\hline 9 & $\mathrm{H}$ & -100.8480 & 24.6740 & 42.3980 & $\mathrm{H}$ & 1 & $\star \star \star \star \star$ & \\
\hline \multicolumn{9}{|c|}{0.0000} \\
\hline 10 & $\mathrm{H}$ & -100.1230 & 23.6530 & 41.1490 & $\mathrm{H}$ & 1 & $\star \star \star \star *$ & \\
\hline \multicolumn{9}{|c|}{0.0000} \\
\hline 11 & $\mathrm{C}$ & -98.9850 & 23.7760 & 43.0390 & C. 3 & 1 & $\star \star \star \star *$ & \\
\hline \multicolumn{9}{|c|}{0.2700} \\
\hline 12 & $\mathrm{C}$ & -97.8950 & 22.8580 & 42.4490 & C. 3 & 1 & $\star \star \star \star *$ & \\
\hline \multicolumn{9}{|c|}{0.2800} \\
\hline 13 & $\mathrm{H}$ & -97.1870 & 23.4390 & 41.8440 & $\mathrm{H}$ & 1 & $\star \star \star \star \star$ & \\
\hline \multicolumn{9}{|c|}{0.0000} \\
\hline 14 & 0 & -97.1940 & 22.2020 & 43.5160 & 0.3 & 1 & $\star \star \star \star *$ & - \\
\hline \multicolumn{9}{|c|}{0.3630} \\
\hline 15 & $\mathrm{C}$ & -96.1700 & 21.3380 & 43.1590 & C.ar & 1 & $\star \star \star \star *$ & \\
\hline \multicolumn{9}{|c|}{0.0830} \\
\hline 16 & $\mathrm{H}$ & -98.3730 & 22.0950 & 41.8230 & $\mathrm{H}$ & 1 & $\star \star \star \star \star$ & \\
\hline \multicolumn{9}{|c|}{0.0000} \\
\hline 17 & $\mathrm{H}$ & -99.5730 & 23.2290 & 43.7790 & $\mathrm{H}$ & 1 & $\star \star \star \star *$ & \\
\hline 0.0000 & & & & & & & & \\
\hline 18 & S & -98.3440 & 25.2190 & 45.3120 & S. 3 & 1 & $\star \star \star \star$ & \\
\hline 0.5380 & & & & & & & & \\
\hline 19 & $\mathrm{C}$ & -96.8360 & 26.0900 & 45.6220 & C.ar & 1 & $\star \star \star \star *$ & \\
\hline 0.0070 & & & & & & & & \\
\hline 20 & $\mathrm{O}$ & -99.4340 & 26.1100 & 45.6600 & 0.3 & 1 & $\star \star \star \star$ & - \\
\hline 0.2150 & & & & & & & & \\
\hline 21 & O & -98.2370 & 23.9180 & 45.9400 & 0.3 & 1 & $\star \star \star \star \star$ & - \\
\hline 0.2150 & & & & & & & & \\
\hline 22 & $\mathrm{C}$ & -96.7050 & 26.8480 & 46.7900 & C.ar & 1 & $\star \star \star \star$ & - \\
\hline 0.1500 & & & & & & & & \\
\hline 23 & $\mathrm{H}$ & -97.5030 & 26.9220 & 47.5240 & $\mathrm{H}$ & 1 & $\star \star \star \star *$ & \\
\hline 0.1500 & & & & & & & & \\
\hline 24 & $\mathrm{C}$ & -95.5050 & 27.5250 & 47.0090 & C.ar & 1 & $\star \star \star \star \star$ & \\
\hline 0.0860 & & & & & & & & \\
\hline 25 & $\mathrm{C}$ & -94.4830 & 27.4640 & 46.0620 & C.ar & 1 & $\star \star \star \star *$ & \\
\hline 0.1170 & & & & & & & & \\
\hline 26 & $\mathrm{C}$ & -94.6160 & 26.7420 & 44.8880 & C.ar & 1 & $\star \star \star \star \star$ & - \\
\hline 0.1500 & & & & & & & & \\
\hline $\begin{array}{r}27 \\
0.1500^{27}\end{array}$ & $\mathrm{H}$ & -93.8250 & 26.7110 & 44.1460 & $\mathrm{H}$ & 1 & $\star \star \star \star *$ & \\
\hline
\end{tabular}




\begin{tabular}{|c|c|c|c|c|c|c|c|c|}
\hline 28 & $\mathrm{C}$ & -95.8110 & 26.0530 & 44.6640 & C.ar & 1 & $\star \star \star \star$ & - \\
\hline \multicolumn{9}{|c|}{0.1500} \\
\hline 29 & $\mathrm{H}$ & -95.9310 & 25.4960 & 43.7380 & $\mathrm{H}$ & 1 & $\star \star \star \star *$ & \\
\hline \multicolumn{9}{|c|}{0.1500} \\
\hline 30 & $\mathrm{C}$ & -95.0420 & 28.3710 & 48.1020 & C. 2 & 1 & $\star \star \star \star *$ & \\
\hline \multicolumn{9}{|c|}{0.4840} \\
\hline 31 & 0 & -95.6460 & 28.6730 & 49.1150 & 0.2 & 1 & $\star \star \star \star$ & - \\
\hline \multicolumn{9}{|c|}{0.5700} \\
\hline 32 & $\mathrm{C}$ & -93.6480 & 28.8140 & 47.7460 & C. 2 & 1 & $\star \star \star \star$ & \\
\hline \multicolumn{9}{|c|}{0.6300} \\
\hline 33 & 0 & -92.8570 & 29.5210 & 48.3640 & 0.2 & 1 & 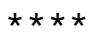 & - \\
\hline \multicolumn{9}{|l|}{0.5700} \\
\hline 34 & $\mathrm{~N}$ & -93.3920 & 28.2290 & 46.4970 & N.am & 1 & $\star \star \star \star *$ & - \\
\hline \multicolumn{9}{|l|}{0.4770} \\
\hline 35 & $\mathrm{C}$ & -92.1700 & 28.4120 & 45.7490 & C. 3 & 1 & $\star \star \star \star \star$ & \\
\hline \multicolumn{9}{|l|}{0.4440} \\
\hline 36 & $\mathrm{H}$ & -91.7580 & 27.4330 & 45.4770 & $\mathrm{H}$ & 1 & $\star \star \star \star *$ & \\
\hline \multicolumn{9}{|l|}{0.0000} \\
\hline 37 & $\mathrm{H}$ & -92.4030 & 28.9240 & 44.8090 & $\mathrm{H}$ & 1 & 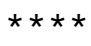 & \\
\hline \multicolumn{9}{|l|}{0.0000} \\
\hline 38 & $\mathrm{C}$ & -91.1250 & 29.1730 & 46.5320 & C.ar & 1 & $\star \star \star \star *$ & \\
\hline \multicolumn{9}{|l|}{0.1670} \\
\hline 39 & $\mathrm{C}$ & -96.3160 & 19.9850 & 42.8250 & C.ar & 1 & $\star \star \star \star *$ & - \\
\hline \multicolumn{9}{|l|}{0.1500} \\
\hline 40 & $\mathrm{H}$ & -97.2830 & 19.4970 & 42.8180 & $\mathrm{H}$ & 1 & $\star \star \star \star *$ & \\
\hline \multicolumn{9}{|l|}{0.1500} \\
\hline 41 & $\mathrm{C}$ & -95.1920 & 19.2260 & 42.4880 & C.ar & 1 & $\star \star \star \star *$ & - \\
\hline \multicolumn{9}{|l|}{0.1500} \\
\hline 42 & $\mathrm{H}$ & -95.3060 & 18.1780 & 42.2270 & $\mathrm{H}$ & 1 & 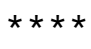 & \\
\hline \multicolumn{9}{|c|}{0.1500} \\
\hline 43 & $\mathrm{C}$ & -93.9260 & 19.8080 & 42.4840 & C.ar & 1 & 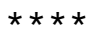 & - \\
\hline \multicolumn{9}{|l|}{0.1500} \\
\hline 44 & $\mathrm{H}$ & -93.0540 & 19.2140 & 42.2230 & $\mathrm{H}$ & 1 & 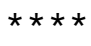 & \\
\hline \multicolumn{9}{|l|}{0.1500} \\
\hline 45 & $\mathrm{C}$ & -93.7790 & 21.1540 & 42.8140 & C.ar & 1 & $\star \star \star \star *$ & - \\
\hline 0.1500 & & & & & & & & \\
\hline 46 & $\mathrm{H}$ & -92.7930 & 21.6120 & 42.8100 & $\mathrm{H}$ & 1 & $\star \star \star \star$ & \\
\hline 0.1500 & & & & & & & & \\
\hline 47 & $\mathrm{C}$ & -94.8980 & 21.9160 & 43.1490 & C.ar & 1 & $\star \star \star \star \star$ & - \\
\hline 0.1500 & & & & & & & & \\
\hline 48 & $\mathrm{H}$ & -94.7830 & 22.9660 & 43.4030 & $\mathrm{H}$ & 1 & $\star \star \star \star$ & \\
\hline 0.1500 & & & & & & & & \\
\hline 49 & $\mathrm{C}$ & -90.1780 & 28.4960 & 47.3080 & C.ar & 1 & $\star \star \star \star \star$ & - \\
\hline 0.1500 & & & & & & & & \\
\hline 50 & $\mathrm{H}$ & -90.1780 & 27.4100 & 47.3560 & $\mathrm{H}$ & 1 & 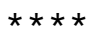 & \\
\hline 0.1500 & & & & & & & & \\
\hline 51 & $\mathrm{C}$ & -89.2510 & 29.2350 & 48.0380 & C.ar & 1 & 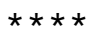 & - \\
\hline 0.1500 & & & & & & & & \\
\hline 52 & $\mathrm{H}$ & -88.5080 & 28.7320 & 48.6490 & $\mathrm{H}$ & 1 & $\star \star \star \star *$ & \\
\hline 0.1500 & & & & & & & & \\
\hline 53 & $\mathrm{C}$ & -89.2950 & 30.6240 & 47.9830 & C.ar & 1 & $\star \star \star \star *$ & - \\
\hline 0.1500 & & & & & & & & \\
\hline 54 & $\mathrm{H}$ & -88.5950 & 31.2360 & 48.5380 & $\mathrm{H}$ & 1 & $\star \star \star \star *$ & \\
\hline 0.1500 & & & & & & & & \\
\hline $0.5000^{55}$ & C & -90.2700 & 31.2140 & 47.1920 & C.ar & 1 & $\star \star \star \star ~$ & \\
\hline
\end{tabular}




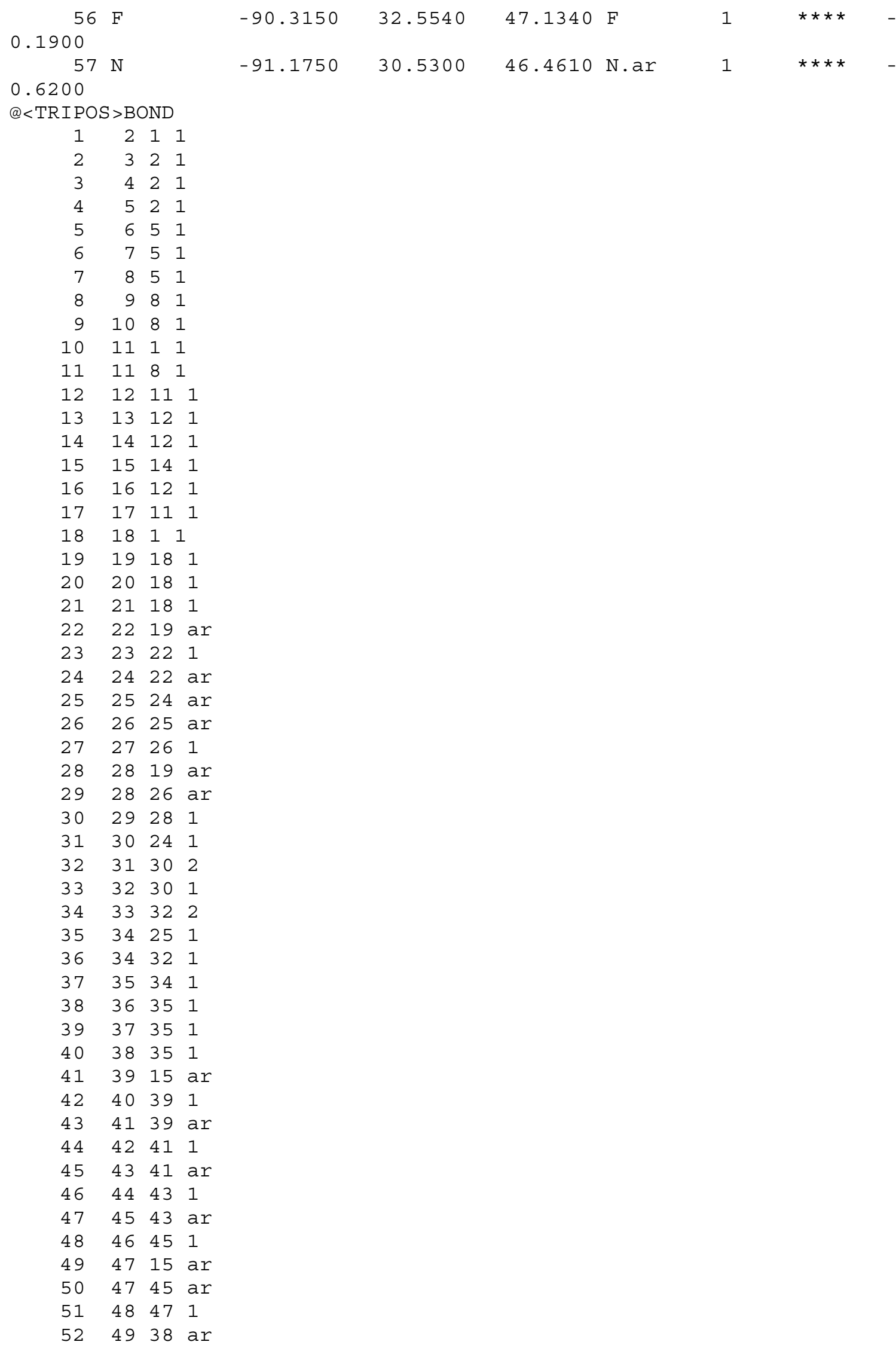




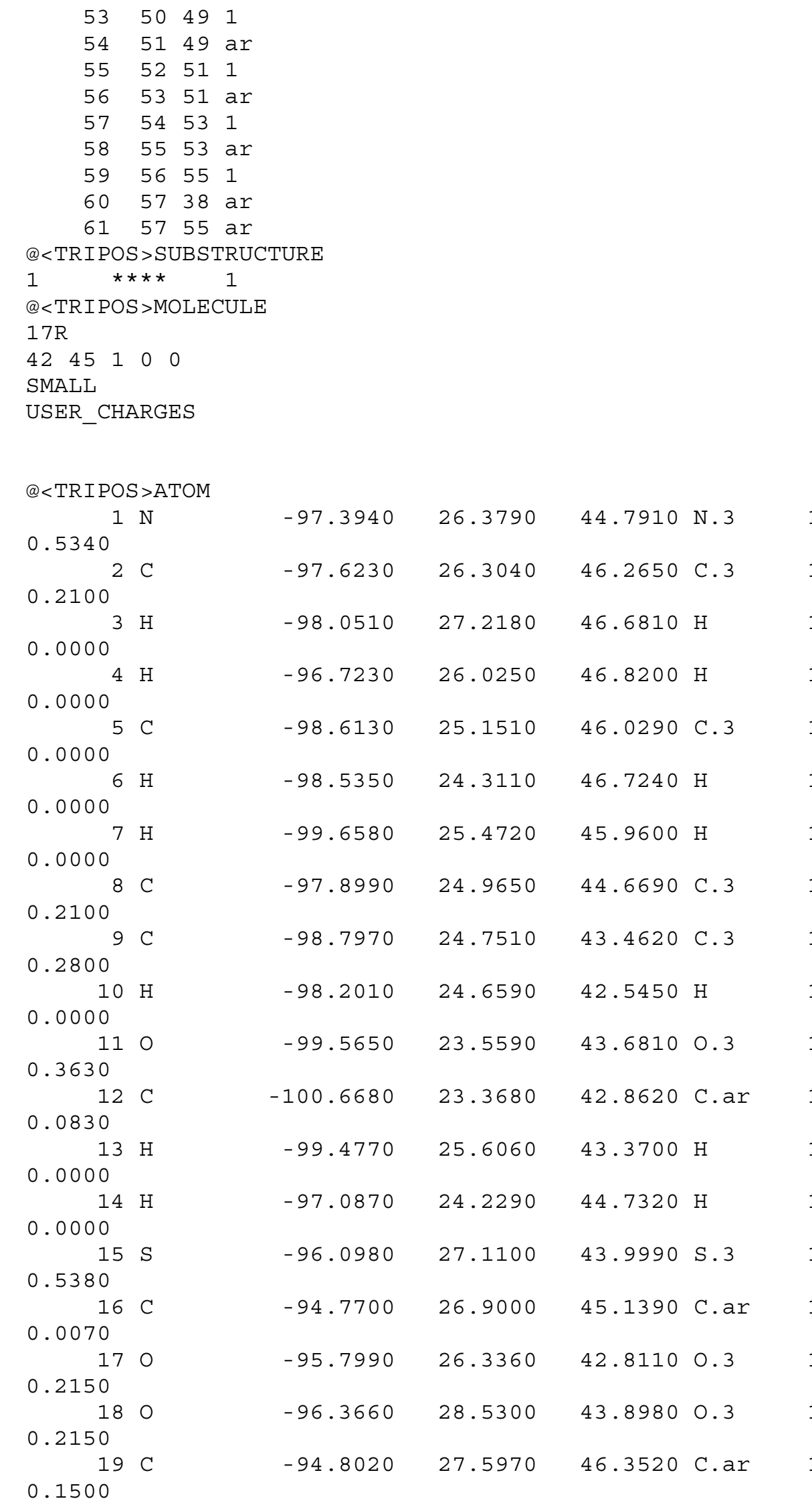




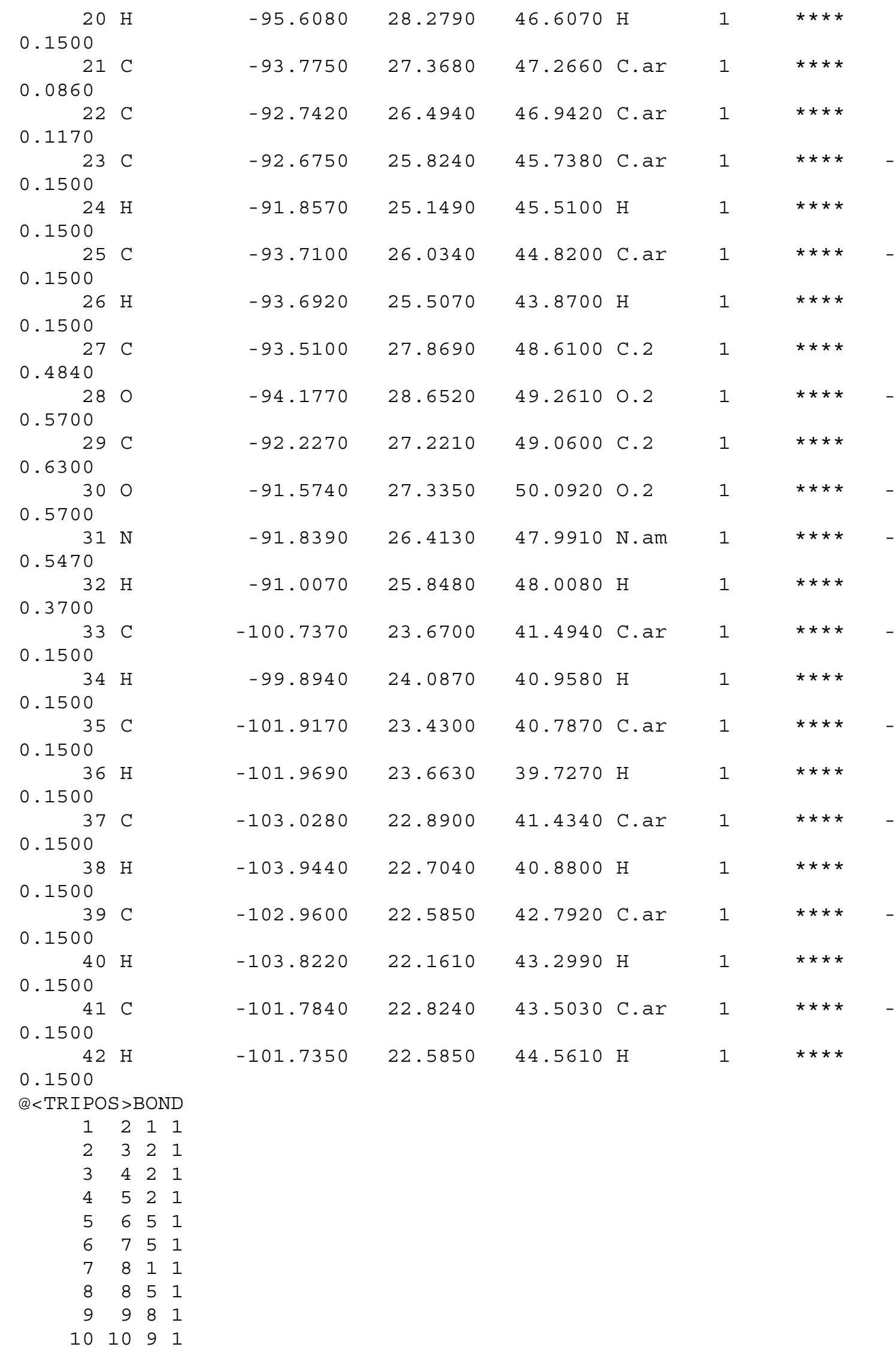




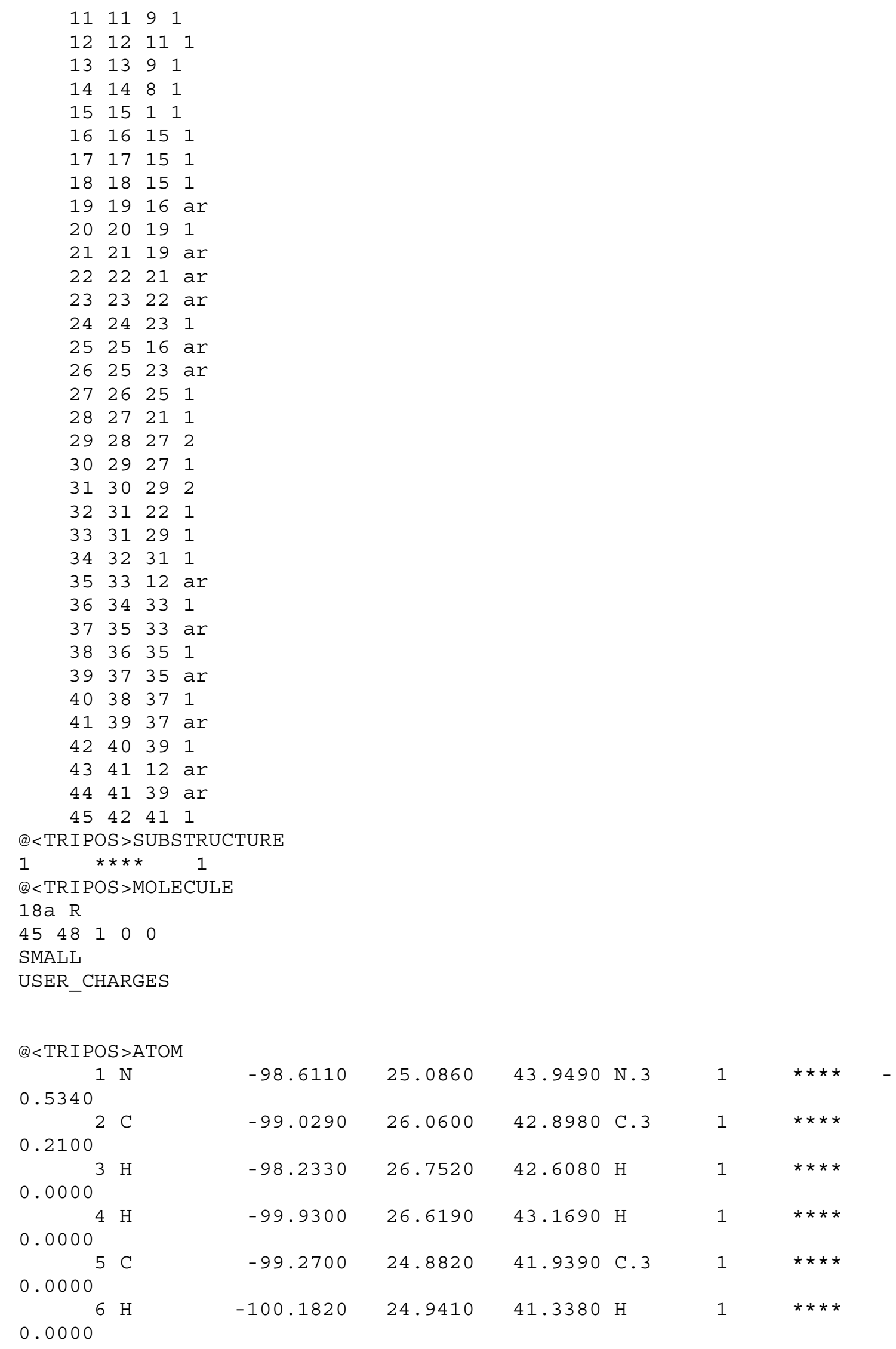




\begin{tabular}{|c|c|c|c|c|c|}
\hline 7 & $\mathrm{H}$ & -98.4180 & 24.6600 & 41.2860 & $\mathrm{H}$ \\
\hline 0.0000 & & & & & \\
\hline 8 & $\mathrm{C}$ & -99.3600 & 24.0080 & 43.2130 & C. 3 \\
\hline 0.2100 & & & & & \\
\hline 9 & $\mathrm{C}$ & -98.6190 & 22.6830 & 43.1730 & C. 3 \\
\hline 0.2800 & & & & & \\
\hline 10 & $\mathrm{H}$ & -98.9430 & 22.0810 & 42.3160 & $\mathrm{H}$ \\
\hline 0.0000 & & & & & \\
\hline 11 & $\mathrm{O}$ & -97.2120 & 22.9590 & 43.0850 & 0.3 \\
\hline 0.3630 & & & & & \\
\hline 12 & $\mathrm{C}$ & -96.3700 & 21.8690 & 42.9310 & C.ar \\
\hline 0.0830 & & & & & \\
\hline 13 & $\mathrm{H}$ & -98.8210 & 22.1350 & 44.1010 & $\mathrm{H}$ \\
\hline 0.0000 & & & & & \\
\hline 14 & $\mathrm{H}$ & -100.3950 & 23.9100 & 43.5660 & $\mathrm{H}$ \\
\hline 0.0000 & & & & & \\
\hline 15 & $S$ & -98.5190 & 25.3630 & 45.6090 & S. 3 \\
\hline 0.5380 & & & & & \\
\hline 16 & $\mathrm{C}$ & -96.9970 & 26.2380 & 45.7780 & C.ar \\
\hline 0.0070 & & & & & \\
\hline 17 & 0 & -99.5970 & 26.2550 & 45.9820 & 0.3 \\
\hline 0.2150 & & & & & \\
\hline 18 & O & -98.3620 & 24.0870 & 46.2780 & 0.3 \\
\hline 0.2150 & & & & & \\
\hline 19 & C & -96.7720 & 26.9920 & 46.9320 & C.ar \\
\hline 0.1500 & & & & & \\
\hline 20 & $\mathrm{H}$ & -97.5120 & 27.0720 & 47.7230 & $\mathrm{H}$ \\
\hline 0.1500 & & & & & \\
\hline $\begin{array}{r}21 \\
0.0860\end{array}$ & C & -95.5370 & 27.6220 & 47.0770 & C.ar \\
\hline $\begin{array}{r}22 \\
0.1170^{2}\end{array}$ & C & -94.5770 & 27.5240 & 46.0700 & C.ar \\
\hline $0.1500^{23}$ & C & -94.8060 & 26.8110 & 44.9060 & C.ar \\
\hline 24 & $\mathrm{H}$ & -94.0550 & 26.7410 & 44.1260 & $\mathrm{H}$ \\
\hline 0.1500 & & & & & \\
\hline $0.1500^{25}$ & C & -96.0340 & 26.1590 & 44.7590 & C.ar \\
\hline 26 & $\mathrm{H}$ & -96.2240 & 25.5790 & 43.8610 & $\mathrm{H}$ \\
\hline 0.1500 & & & & & \\
\hline 27 & $\mathrm{C}$ & -94.9680 & 28.4320 & 48.1470 & C. 2 \\
\hline 0.4840 & & & & & \\
\hline 28 & $\mathrm{O}$ & -95.4940 & 28.7470 & 49.1990 & 0.2 \\
\hline 0.5700 & & & & & \\
\hline 29 & $\mathrm{C}$ & -93.5790 & 28.8180 & 47.7090 & C. 2 \\
\hline 0.6300 & & & & & \\
\hline 30 & $\mathrm{O}$ & -92.7350 & 29.5130 & 48.2680 & 0.2 \\
\hline 0.5700 & & & & & \\
\hline 31 & $\mathrm{~N}$ & -93.4280 & 28.2290 & 46.4430 & N. am \\
\hline 0.4770 & & & & & \\
\hline 32 & $\mathrm{C}$ & -92.2470 & 28.3280 & 45.6250 & C. 3 \\
\hline 0.3000 & & & & & \\
\hline 33 & $\mathrm{H}$ & -91.5440 & 29.0500 & 46.0520 & $\mathrm{H}$ \\
\hline 0.0000 & & & & & \\
\hline $0.0000^{34}$ & $\mathrm{H}$ & -91.7600 & 27.3490 & 45.5710 & $\mathrm{H}$ \\
\hline
\end{tabular}




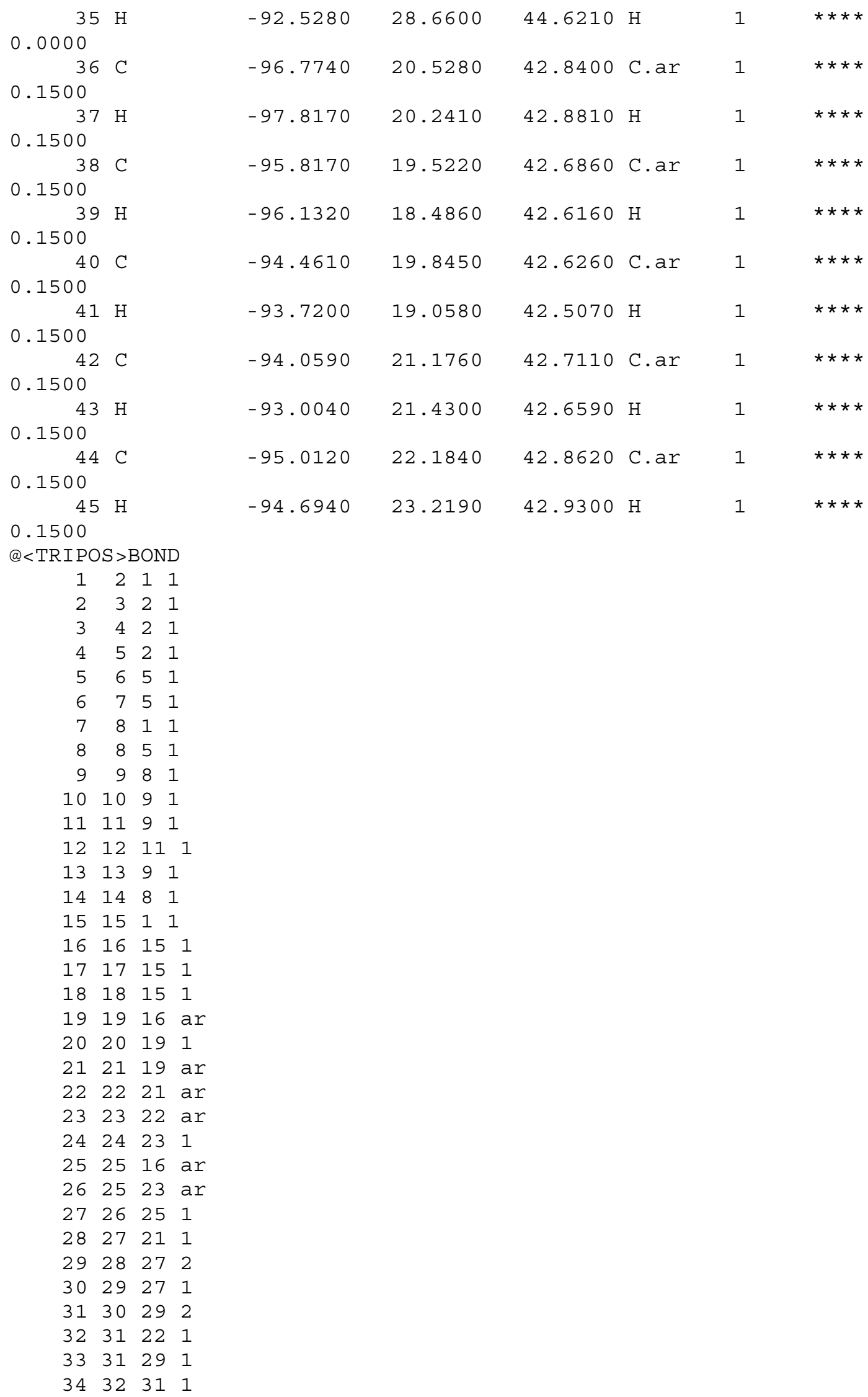




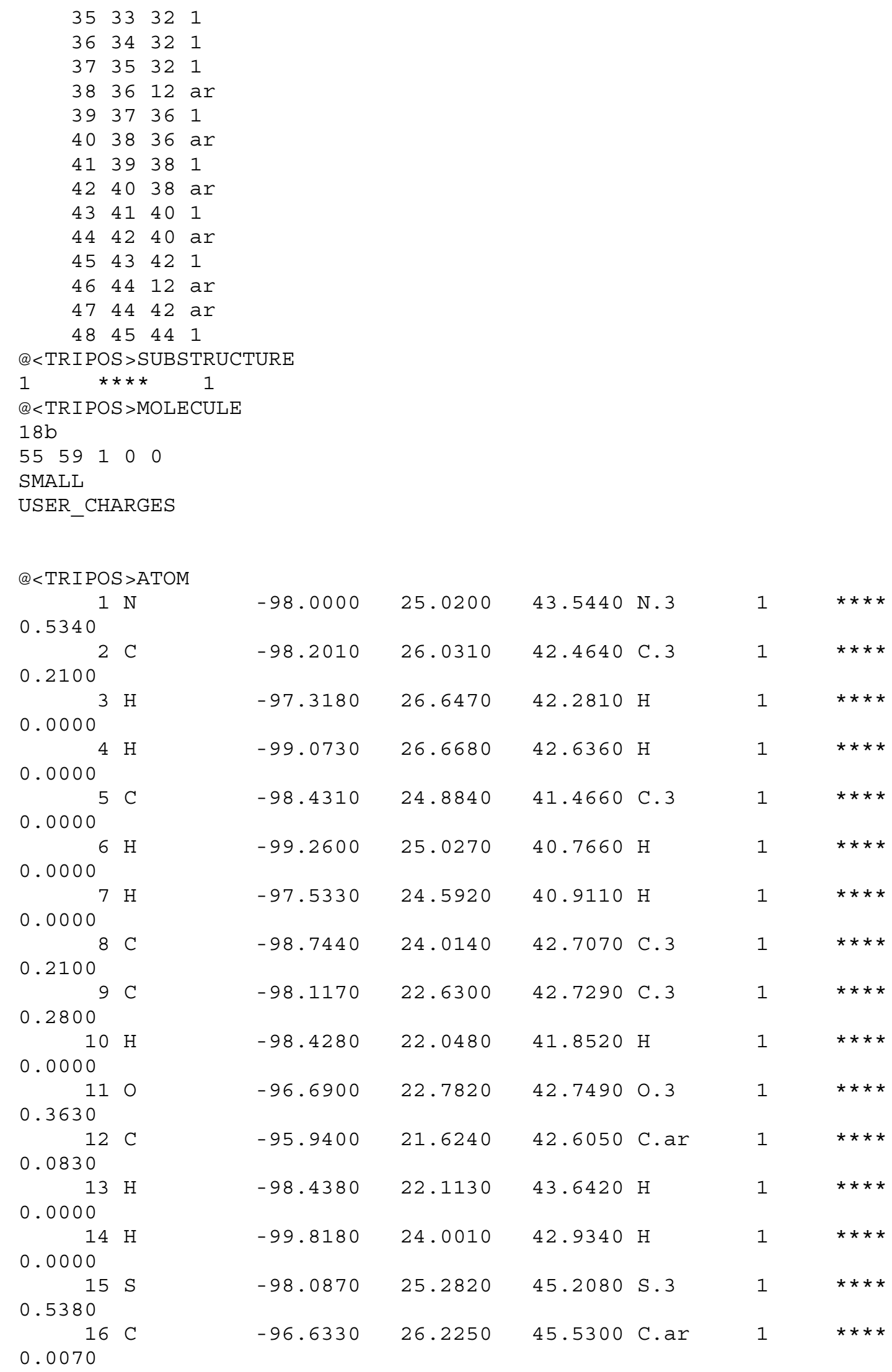




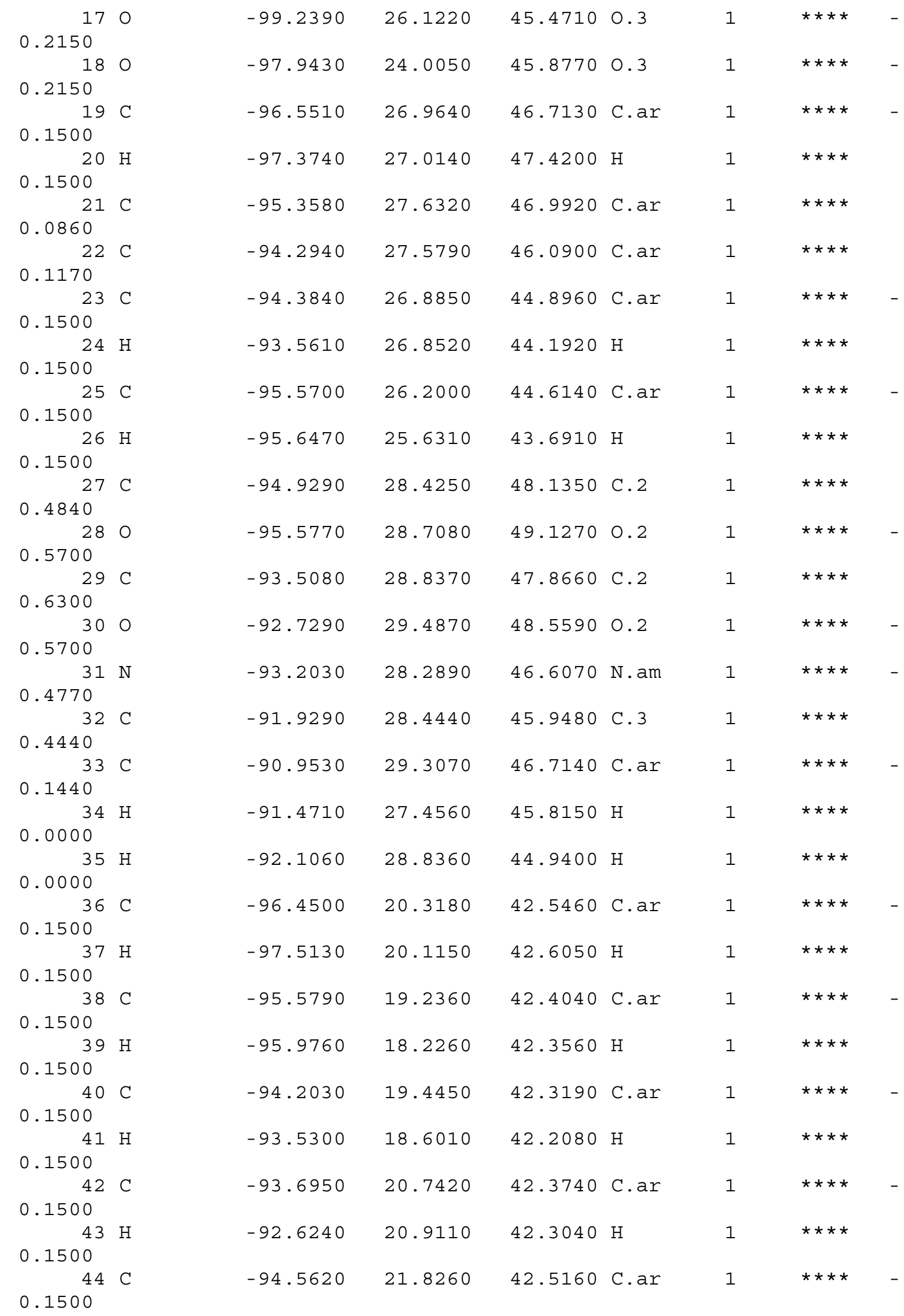




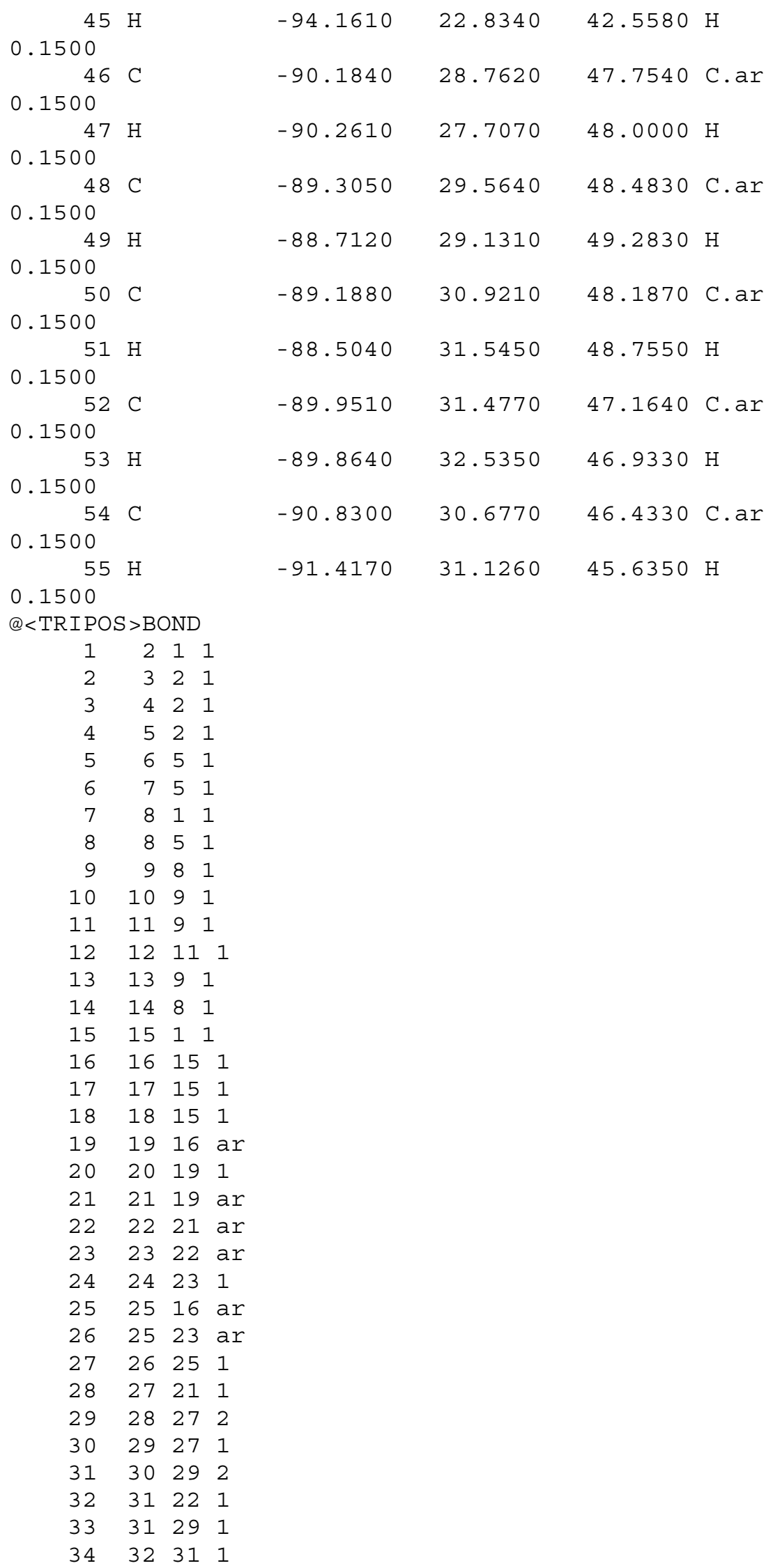




\begin{tabular}{|c|c|c|c|c|c|c|c|c|c|}
\hline & & & & & & & & & \\
\hline 36 & 34 & 32 & 1 & & & & & & \\
\hline 37 & 35 & 32 & 1 & & & & & & \\
\hline 38 & 36 & 12 & ar & & & & & & \\
\hline 39 & 37 & 36 & 1 & & & & & & \\
\hline 40 & 38 & 36 & ar & & & & & & \\
\hline 41 & 39 & 38 & 1 & & & & & & \\
\hline 42 & 40 & 38 & ar & & & & & & \\
\hline 43 & 41 & 40 & 1 & & & & & & \\
\hline 44 & 42 & 40 & ar & & & & & & \\
\hline 45 & 43 & 42 & 1 & & & & & & \\
\hline 46 & 44 & 12 & ar & & & & & & \\
\hline 47 & 44 & 42 & ar & & & & & & \\
\hline 48 & 45 & 44 & 1 & & & & & & \\
\hline 49 & 46 & 33 & ar & & & & & & \\
\hline 50 & 47 & 46 & 1 & & & & & & \\
\hline 51 & 48 & 46 & ar & & & & & & \\
\hline 52 & 49 & 48 & 1 & & & & & & \\
\hline 53 & 50 & 48 & ar & & & & & & \\
\hline 54 & 51 & 50 & 1 & & & & & & \\
\hline 55 & 52 & 50 & ar & & & & & & \\
\hline 56 & 53 & 52 & 1 & & & & & & \\
\hline 57 & 54 & 33 & ar & & & & & & \\
\hline 58 & 54 & 52 & ar & & & & & & \\
\hline 59 & 55 & 54 & 1 & & & & & & \\
\hline$@<$ TRIPO & $\mathrm{S}>\mathrm{SU}$ & JBST & RUCTURE & & & & & & \\
\hline $1 \quad$ * & $\star \star * *$ & & 1 & & & & & & \\
\hline$@<$ TRIPO & $\mathrm{S}>\mathrm{MO}$ & LEC & ULE & & & & & & \\
\hline $18 \mathrm{C} \mathrm{R}$ & & & & & & & & & \\
\hline $\begin{array}{lll}59 & 63 & 1\end{array}$ & 00 & & & & & & & & \\
\hline SMALL & & & & & & & & & \\
\hline USER_CH & $A R G E$ & & & & & & & & \\
\hline$@<$ TRIPO & $\mathrm{S}>\mathrm{AT}$ & & & & & & & & \\
\hline 1 & $\mathrm{~N}$ & & -98.3830 & 25.1060 & 43.8300 & N. 3 & 1 & $\star * * *$ & - \\
\hline 0.5340 & & & & & & & & & \\
\hline 2 & C & & -98.6520 & 26.1680 & 42.8140 & C. 3 & 1 & $\star \star \star \star *$ & \\
\hline 0.2100 & & & & & & & & & \\
\hline 3 & $\mathrm{H}$ & & -97.7660 & 26.7560 & 42.5630 & $\mathrm{H}$ & 1 & $\star * \star *$ & \\
\hline 0.0000 & & & & & & & & & \\
\hline $0.0000^{4}$ & $\mathrm{H}$ & & -99.4730 & 26.8310 & 43.1010 & $\mathrm{H}$ & 1 & $\star \star \star * *$ & \\
\hline 5 & $\mathrm{C}$ & & -99.0340 & 25.0720 & 41.8050 & C. 3 & 1 & $* * * *$ & \\
\hline 0.0000 & & & & & & & & & \\
\hline 6 & $\mathrm{H}$ & & -99.9190 & 25.2770 & 41.1980 & $\mathrm{H}$ & 1 & $\star * * *$ & \\
\hline 0.0000 & & & & & & & & & \\
\hline${ }^{7}$ & $\mathrm{H}$ & & -98.2080 & 24.7640 & 41.1540 & $\mathrm{H}$ & 1 & $\star \star \star * *$ & \\
\hline 8 & C & & -99.2600 & 24.1690 & 43.0410 & C. 3 & 1 & $\star \star \star *$ & \\
\hline 0.2100 & & & & & & & & & \\
\hline 9 & C & & -98.7050 & 22.7570 & 42.9510 & C. 3 & 1 & $\star * * *$ & \\
\hline 0.2800 & & & & & & & & & \\
\hline 10 & $\mathrm{H}$ & & -98.8500 & 22.3470 & 41.9450 & $\mathrm{H}$ & 1 & $\star \star * * *$ & \\
\hline 0.0000 & & & & & & & & & \\
\hline 11 & 0 & & -97.3070 & 22.8000 & 43.2750 & 0.3 & 1 & $\star \star \star \star *$ & - \\
\hline
\end{tabular}




\begin{tabular}{|c|c|c|c|c|c|c|c|}
\hline 12 & $\mathrm{C}$ & -96.5830 & 21.6360 & 43.0650 & C.ar & 1 & $\star \star \star \star$ \\
\hline \multicolumn{8}{|c|}{0.0830} \\
\hline 13 & $\mathrm{H}$ & -99.2250 & 22.1260 & 43.6810 & $\mathrm{H}$ & 1 & $\star \star \star \star$ \\
\hline \multicolumn{8}{|l|}{0.0000} \\
\hline 14 & $\mathrm{H}$ & -100.3050 & 24.1960 & 43.3770 & $\mathrm{H}$ & 1 & $\star \star \star \star *$ \\
\hline \multicolumn{8}{|c|}{0.0000} \\
\hline 15 & $S$ & -98.2960 & 25.3050 & 45.5030 & S. 3 & 1 & 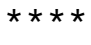 \\
\hline \multicolumn{8}{|c|}{0.5380} \\
\hline 16 & $\mathrm{C}$ & -96.8190 & 26.2460 & 45.7090 & C.ar & 1 & $\star \star \star \star *$ \\
\hline \multicolumn{8}{|c|}{0.0070} \\
\hline 17 & O & -99.4140 & 26.1270 & 45.9210 & 0.3 & 1 & 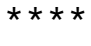 \\
\hline \multicolumn{8}{|c|}{0.2150} \\
\hline 18 & O & -98.0760 & 24.0070 & 46.1070 & 0.3 & 1 & 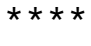 \\
\hline \multicolumn{8}{|c|}{0.2150} \\
\hline 19 & $\mathrm{C}$ & -96.6460 & 26.9930 & 46.8760 & C.ar & 1 & 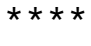 \\
\hline \multicolumn{8}{|c|}{0.1500} \\
\hline $\begin{array}{r}20 \\
0.1500^{2}\end{array}$ & \multicolumn{6}{|c|}{0.1500} & 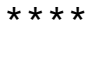 \\
\hline $\begin{array}{r}21 \\
0.0860\end{array}$ & C & -95.4310 & 27.6530 & 47.0630 & C.ar & 1 & $\star \star \star * *$ \\
\hline \multicolumn{8}{|c|}{0.1170} \\
\hline \multicolumn{8}{|c|}{0.1500} \\
\hline \multicolumn{8}{|c|}{0.1500} \\
\hline \multicolumn{8}{|c|}{0.1500} \\
\hline $0.1500^{26}$ & \multicolumn{6}{|c|}{0.1500} & $\star * \star *$ \\
\hline \multicolumn{8}{|c|}{0.4840} \\
\hline \multicolumn{8}{|c|}{0.5700} \\
\hline $0.6300^{29}$ & \multicolumn{6}{|c|}{0.6300} & $\star \star \star \star$ \\
\hline \multicolumn{8}{|c|}{0.5700} \\
\hline $\begin{array}{r}31 \\
0.4770^{3}\end{array}$ & \multicolumn{6}{|c|}{0.4770} & $\star \star \star \star$ \\
\hline \multicolumn{8}{|c|}{0.4440} \\
\hline \multicolumn{8}{|c|}{0.1440} \\
\hline \multicolumn{8}{|c|}{0.0000} \\
\hline $0.0000^{35}$ & $\mathrm{H}$ & -92.2970 & 28.9380 & 44.8240 & $\mathrm{H}$ & 1 & $\star \star \star *$ \\
\hline $0.1500^{36}$ & $\mathrm{C}$ & -97.1260 & 20.3580 & 42.8640 & C.ar & 1 & $\star \star \star *$ \\
\hline $0.1500^{37}$ & $\mathrm{H}$ & -98.1970 & 20.1870 & 42.8550 & $\mathrm{H}$ & 1 & $\star \star \star \star *$ \\
\hline $\begin{array}{r}38 \\
0.1500^{38}\end{array}$ & C & -96.2800 & 19.2650 & 42.6650 & C.ar & 1 & $\star \star \star \star$ \\
\hline $0.1500^{39}$ & $\mathrm{H}$ & -96.7030 & 18.2760 & 42.5090 & $\mathrm{H}$ & 1 & $\star \star \star *$ \\
\hline
\end{tabular}




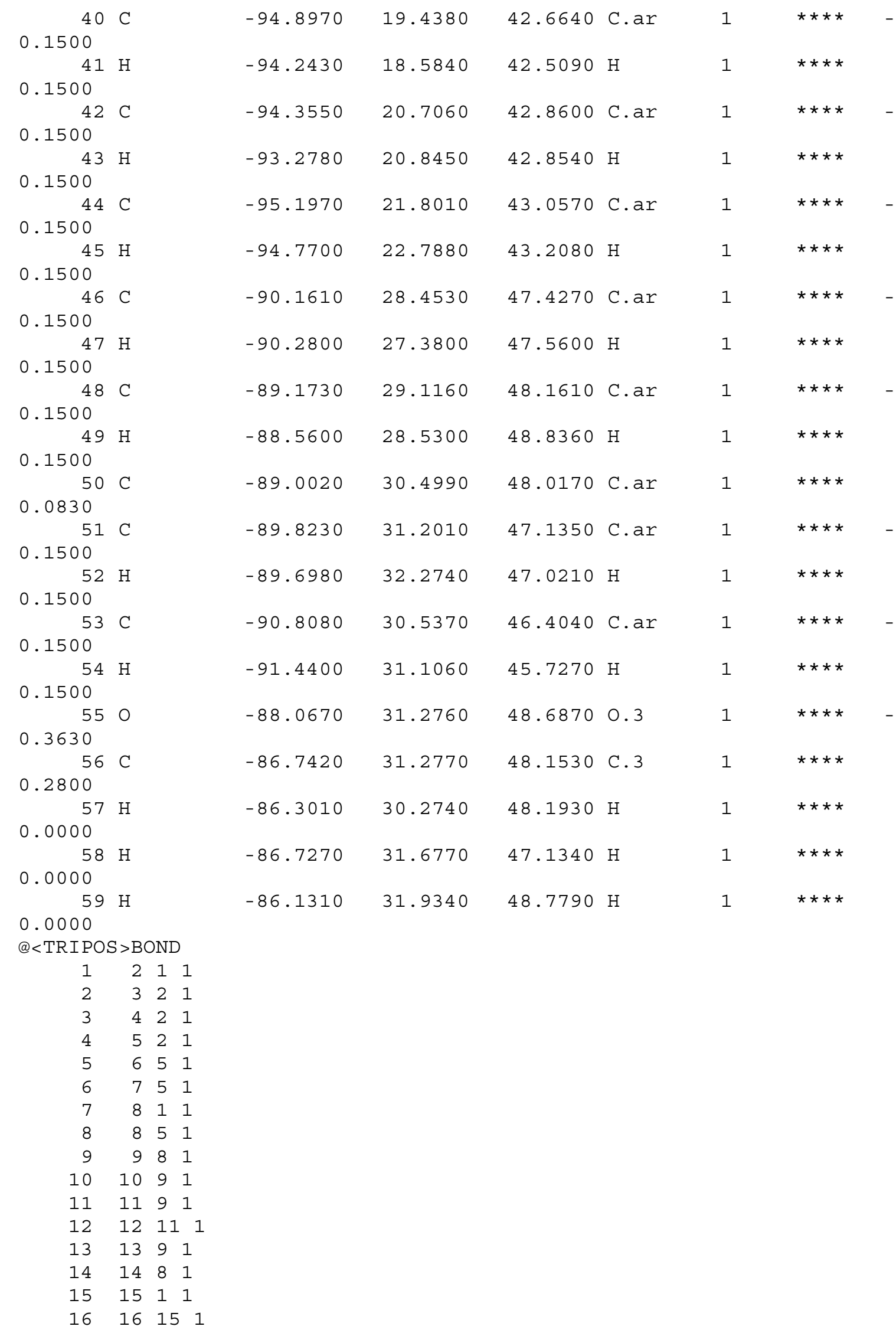




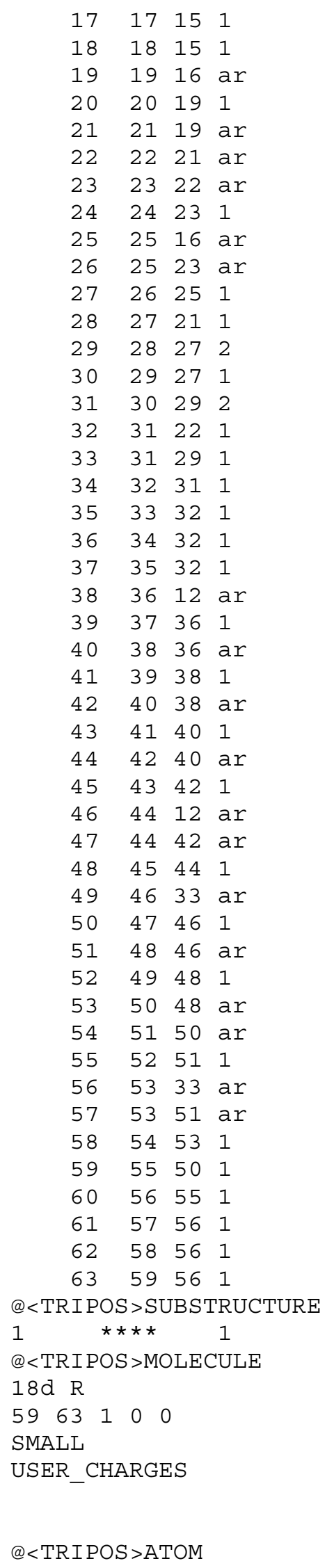




\begin{tabular}{|c|c|c|c|c|c|c|c|c|}
\hline 1 & $\mathrm{~N}$ & -98.5720 & 25.0790 & 43.9660 & N. 3 & 1 & $\star \star \star \star$ & - \\
\hline \multicolumn{9}{|c|}{0.5340} \\
\hline 2 & $\mathrm{C}$ & -98.9250 & 26.1290 & 42.9640 & C. 3 & 1 & $\star \star \star *$ & \\
\hline \multicolumn{9}{|c|}{0.2100} \\
\hline 3 & $\mathrm{H}$ & -98.0770 & 26.7580 & 42.6820 & $\mathrm{H}$ & 1 & $\star \star \star \star$ & \\
\hline \multicolumn{9}{|c|}{0.0000} \\
\hline 4 & $\mathrm{H}$ & -99.7640 & 26.7550 & 43.2840 & $\mathrm{H}$ & 1 & $\star \star \star \star$ & \\
\hline \multicolumn{9}{|c|}{0.0000} \\
\hline 5 & $\mathrm{C}$ & -99.2940 & 25.0190 & 41.9680 & C. 3 & 1 & 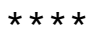 & \\
\hline \multicolumn{9}{|c|}{0.0000} \\
\hline 6 & $\mathrm{H}$ & -100.2100 & 25.1850 & 41.3920 & $\mathrm{H}$ & 1 & 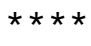 & \\
\hline \multicolumn{9}{|c|}{0.0000} \\
\hline 7 & $\mathrm{H}$ & -98.4810 & 24.7470 & 41.2850 & $\mathrm{H}$ & 1 & $\star \star \star \star$ & \\
\hline \multicolumn{9}{|c|}{0.0000} \\
\hline 8 & $\mathrm{C}$ & -99.4380 & 24.1070 & 43.2090 & C. 3 & 1 & $\star \star \star \star$ & \\
\hline \multicolumn{9}{|c|}{0.2100} \\
\hline 9 & $\mathrm{C}$ & -98.8280 & 22.7190 & 43.0970 & C. 3 & 1 & $\star \star \star \star$ & \\
\hline \multicolumn{9}{|c|}{0.2800} \\
\hline 10 & $\mathrm{H}$ & -98.8470 & 22.3690 & 42.0580 & $\mathrm{H}$ & 1 & $\star \star \star \star$ & \\
\hline \multicolumn{9}{|c|}{0.0000} \\
\hline 11 & O & -97.4770 & 22.7800 & 43.5790 & 0.3 & 1 & $\star \star \star \star ~$ & - \\
\hline \multicolumn{9}{|c|}{0.3630} \\
\hline 12 & $\mathrm{C}$ & -96.6610 & 21.7000 & 43.2790 & C.ar & 1 & $\star \star \star \star$ & \\
\hline \multicolumn{9}{|c|}{0.0830} \\
\hline 13 & $\mathrm{H}$ & -99.4050 & 22.0290 & 43.7240 & $\mathrm{H}$ & 1 & $\star \star \star \star$ & \\
\hline \multicolumn{9}{|c|}{0.0000} \\
\hline 14 & $\mathrm{H}$ & -100.4700 & 24.0920 & 43.5830 & $\mathrm{H}$ & 1 & $\star \star \star \star$ & \\
\hline \multicolumn{9}{|c|}{0.0000} \\
\hline 15 & $\mathrm{~S}$ & -98.4300 & 25.2820 & 45.6350 & S. 3 & 1 & $\star \star \star \star \star$ & \\
\hline \multicolumn{9}{|c|}{0.5380} \\
\hline 16 & $\mathrm{C}$ & -96.9470 & 26.2240 & 45.7870 & C.ar & 1 & 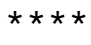 & \\
\hline \multicolumn{9}{|c|}{0.0070} \\
\hline 17 & 0 & -99.5330 & 26.1060 & 46.0880 & 0.3 & 1 & 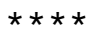 & - \\
\hline \multirow{2}{*}{\multicolumn{8}{|c|}{$\begin{array}{r}0.2150 \\
180\end{array}$}} & \\
\hline & O & -98.1910 & & & & & & - \\
\hline 0.2150 & & & & & & & & \\
\hline 19 & $\mathrm{C}$ & -96.7340 & 26.9730 & 46.9460 & C.ar & 1 & 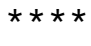 & - \\
\hline 0.1500 & & & & & & & & \\
\hline 20 & $\mathrm{H}$ & -97.4670 & 27.0230 & 47.7450 & $\mathrm{H}$ & 1 & $\star \star \star \star$ & \\
\hline 0.1500 & & & & & & & & \\
\hline 21 & $\mathrm{C}$ & -95.5130 & 27.6360 & 47.0860 & C.ar & 1 & $\star \star \star \star$ & \\
\hline 0.0860 & & & & & & & & \\
\hline 22 & $\mathrm{C}$ & -94.5530 & 27.5670 & 46.0750 & C.ar & 1 & $\star \star \star \star$ & \\
\hline 0.1170 & & & & & & & & \\
\hline 23 & $\mathrm{C}$ & -94.7760 & 26.8640 & 44.9040 & C.ar & 1 & 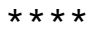 & - \\
\hline 0.1500 & & & & & & & & \\
\hline 24 & $\mathrm{H}$ & -94.0340 & 26.8180 & 44.1160 & $\mathrm{H}$ & 1 & $\star \star \star \star$ & \\
\hline 0.1500 & & & & & & & & \\
\hline 25 & $\mathrm{C}$ & -95.9920 & 26.1880 & 44.7590 & C.ar & 1 & $\star \star \star \star$ & - \\
\hline 0.1500 & & & & & & & & \\
\hline 26 & $\mathrm{H}$ & -96.1740 & 25.6140 & 43.8540 & $\mathrm{H}$ & 1 & $\star \star \star \star \star$ & \\
\hline 0.1500 & & & & & & & & \\
\hline 27 & $\mathrm{C}$ & -94.9630 & 28.4440 & 48.1650 & C. 2 & 1 & $\star \star \star \star$ & \\
\hline 0.4840 & & & & & & & & \\
\hline 28 & O & -95.4980 & 28.7400 & 49.2170 & 0.2 & 1 & $\star \star \star \star *$ & - \\
\hline 0.5700 & 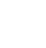 & & & & & & & \\
\hline
\end{tabular}




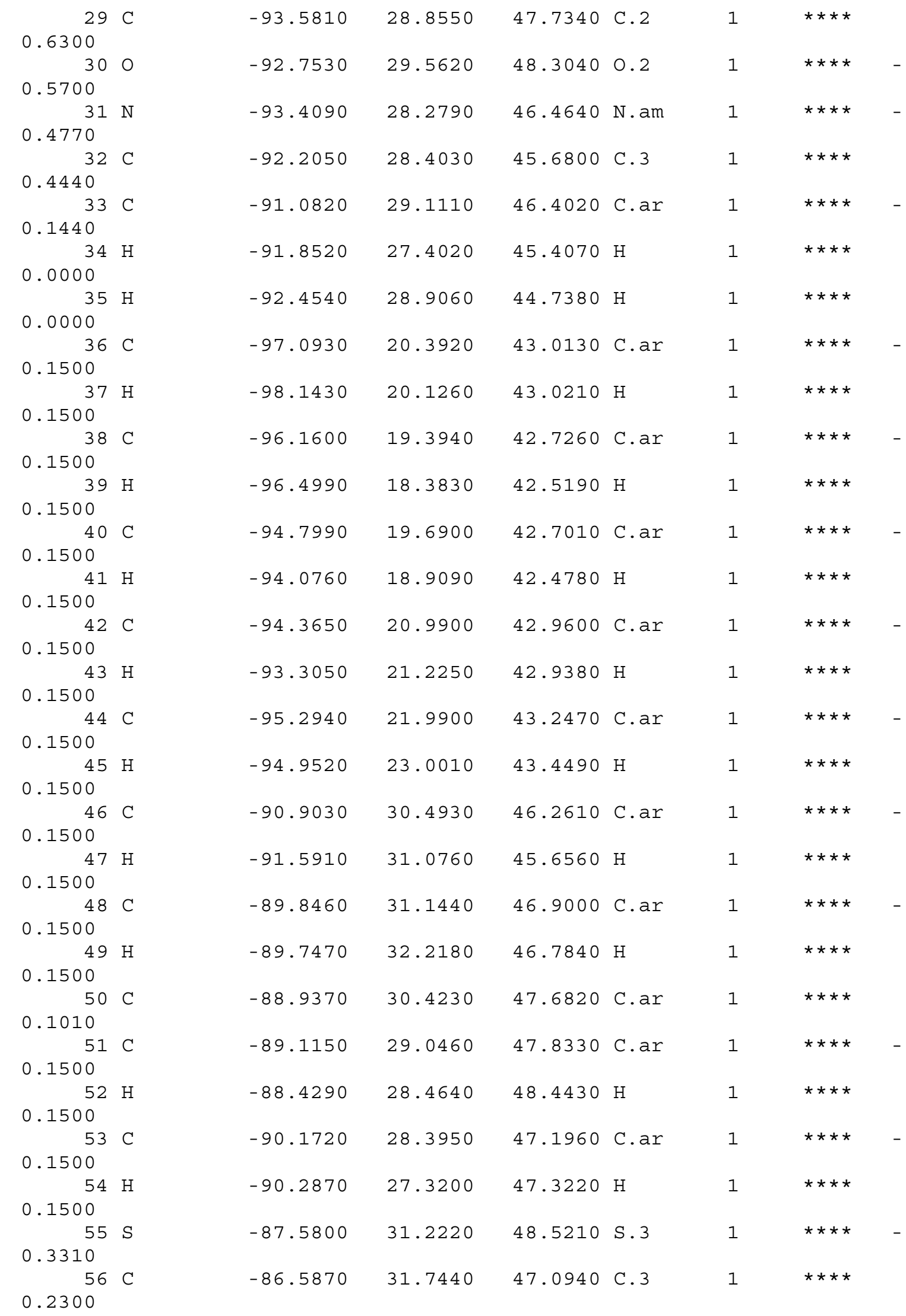




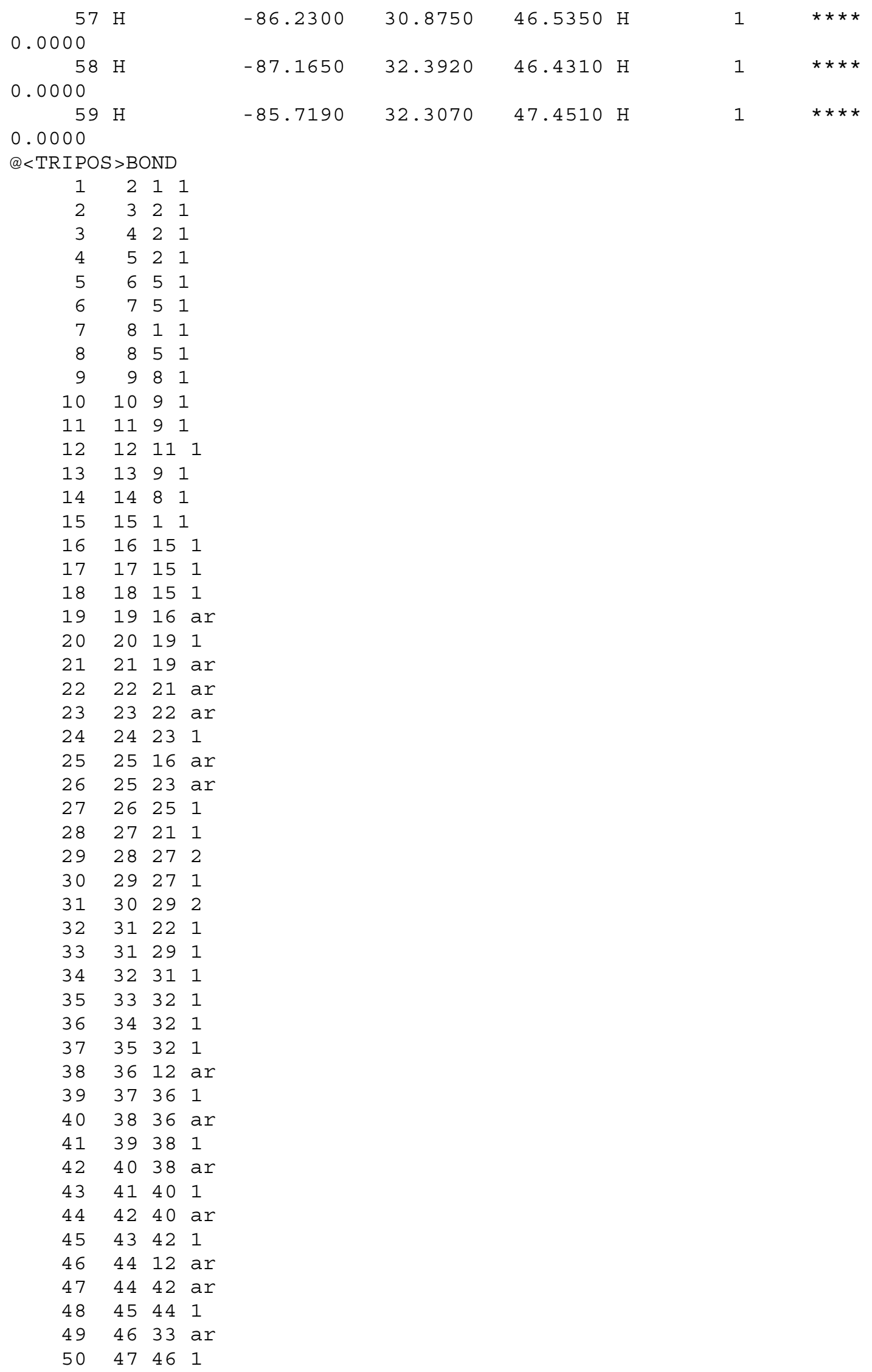




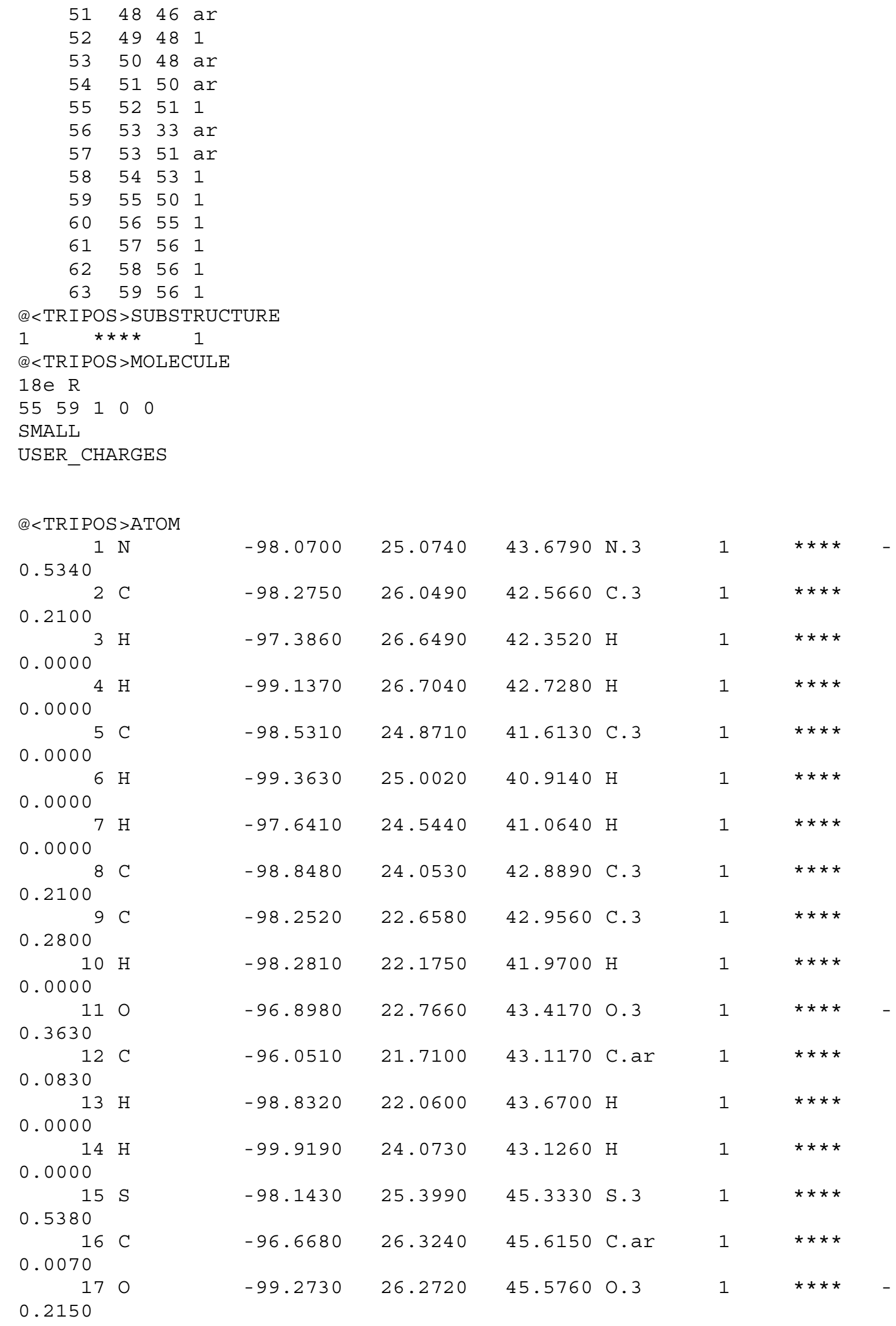




\begin{tabular}{|c|c|c|c|c|c|c|c|c|}
\hline 18 & 0 & -98.0200 & 24.1460 & 46.0510 & 0.3 & 1 & $\star \star \star \star$ & - \\
\hline \multicolumn{9}{|c|}{0.2150} \\
\hline 19 & $\mathrm{C}$ & -96.5380 & 27.0490 & 46.8020 & C.ar & 1 & $\star \star \star \star *$ & - \\
\hline \multicolumn{9}{|c|}{0.1500} \\
\hline 20 & $\mathrm{H}$ & -97.3290 & 27.0890 & 47.5440 & $\mathrm{H}$ & 1 & $\star \star \star \star *$ & \\
\hline \multicolumn{9}{|c|}{0.1500} \\
\hline 21 & $\mathrm{C}$ & -95.3250 & 27.6970 & 47.0470 & C.ar & 1 & $\star \star \star \star$ & \\
\hline \multicolumn{9}{|l|}{0.0860} \\
\hline 22 & $\mathrm{C}$ & -94.2930 & 27.6410 & 46.1090 & C.ar & 1 & $\star \star \star \star$ & \\
\hline \multicolumn{9}{|c|}{0.1170} \\
\hline 23 & $\mathrm{C}$ & -94.4350 & 26.9630 & 44.9110 & C.ar & 1 & 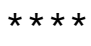 & - \\
\hline \multicolumn{9}{|l|}{0.1500} \\
\hline 24 & $\mathrm{H}$ & -93.6340 & 26.9230 & 44.1810 & $\mathrm{H}$ & 1 & $\star \star \star \star *$ & \\
\hline \multicolumn{9}{|l|}{0.1500} \\
\hline 25 & $\mathrm{C}$ & -95.6390 & 26.2990 & 44.6610 & C.ar & 1 & $\star \star \star \star \star$ & - \\
\hline \multicolumn{9}{|l|}{0.1500} \\
\hline 26 & $\mathrm{H}$ & -95.7560 & 25.7420 & 43.7340 & $\mathrm{H}$ & 1 & $\star \star \star \star *$ & \\
\hline \multicolumn{9}{|l|}{0.1500} \\
\hline 27 & $\mathrm{C}$ & -94.8490 & 28.4740 & 48.1840 & C. 2 & 1 & 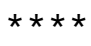 & \\
\hline \multicolumn{9}{|l|}{0.4840} \\
\hline 28 & 0 & -95.4580 & 28.7490 & 49.2010 & 0.2 & 1 & $\star \star \star \star *$ & - \\
\hline \multicolumn{9}{|l|}{0.5700} \\
\hline 29 & $\mathrm{C}$ & -93.4360 & 28.8800 & 47.8680 & C. 2 & 1 & $\star \star \star \star *$ & \\
\hline \multicolumn{9}{|l|}{0.6300} \\
\hline 30 & O & -92.6410 & 29.5620 & 48.5100 & 0.2 & 1 & $\star \star \star \star *$ & - \\
\hline \multicolumn{9}{|l|}{0.5700} \\
\hline 31 & $\mathrm{~N}$ & -93.1760 & 28.3330 & 46.5990 & N.am & 1 & $\star \star \star \star *$ & - \\
\hline \multicolumn{9}{|l|}{0.4770} \\
\hline 32 & $\mathrm{C}$ & -91.9160 & 28.4630 & 45.9090 & C. 3 & 1 & $\star \star \star \star$ & \\
\hline \multicolumn{9}{|l|}{0.4440} \\
\hline 33 & $\mathrm{C}$ & -90.8850 & 29.2580 & 46.6790 & C.ar & 1 & 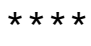 & - \\
\hline \multicolumn{9}{|l|}{0.1440} \\
\hline 34 & $\mathrm{H}$ & -91.5030 & 27.4620 & 45.7290 & $\mathrm{H}$ & 1 & 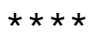 & \\
\hline \multicolumn{9}{|l|}{0.0000} \\
\hline 35 & $\mathrm{H}$ & -92.0990 & 28.8950 & 44.9200 & $\mathrm{H}$ & 1 & $\star \star \star \star *$ & \\
\hline 0.0000 & & & & & & & & \\
\hline 36 & $\mathrm{C}$ & -96.4490 & 20.3950 & 42.8320 & C.ar & 1 & $\star \star \star \star$ & - \\
\hline 0.1500 & & & & & & & & \\
\hline 37 & $\mathrm{H}$ & -97.4930 & 20.1030 & 42.8210 & $\mathrm{H}$ & 1 & $\star \star \star \star \star$ & \\
\hline 0.1500 & & & & & & & & \\
\hline 38 & $\mathrm{C}$ & -95.4890 & 19.4220 & 42.5480 & C.ar & 1 & 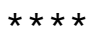 & - \\
\hline 0.1500 & & & & & & & & \\
\hline 39 & $\mathrm{H}$ & -95.7990 & 18.4060 & 42.3260 & $\mathrm{H}$ & 1 & $\star \star \star \star \star$ & \\
\hline 0.1500 & & & & & & & & \\
\hline 40 & $\mathrm{C}$ & -94.1340 & 19.7510 & 42.5450 & C.ar & 1 & 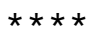 & - \\
\hline 0.1500 & & & & & & & & \\
\hline 41 & $\mathrm{H}$ & -93.3900 & 18.9900 & 42.3240 & $\mathrm{H}$ & 1 & 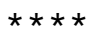 & \\
\hline 0.1500 & & & & & & & & \\
\hline 42 & $\mathrm{C}$ & -93.7360 & 21.0570 & 42.8230 & C.ar & 1 & $\star \star \star \star *$ & - \\
\hline 0.1500 & & & & & & & & \\
\hline 43 & $\mathrm{H}$ & -92.6820 & 21.3180 & 42.8180 & $\mathrm{H}$ & 1 & $\star \star \star \star *$ & \\
\hline 0.1500 & & & & & & & & \\
\hline 44 & $\mathrm{C}$ & -94.6920 & 22.0320 & 43.1080 & C.ar & 1 & $\star \star \star \star *$ & - \\
\hline 0.1500 & & & & & & & & \\
\hline $\begin{array}{r}45 \\
0.1500\end{array}$ & $\mathrm{H}$ & -94.3780 & 23.0480 & 43.3240 & $\mathrm{H}$ & 1 & 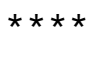 & \\
\hline
\end{tabular}




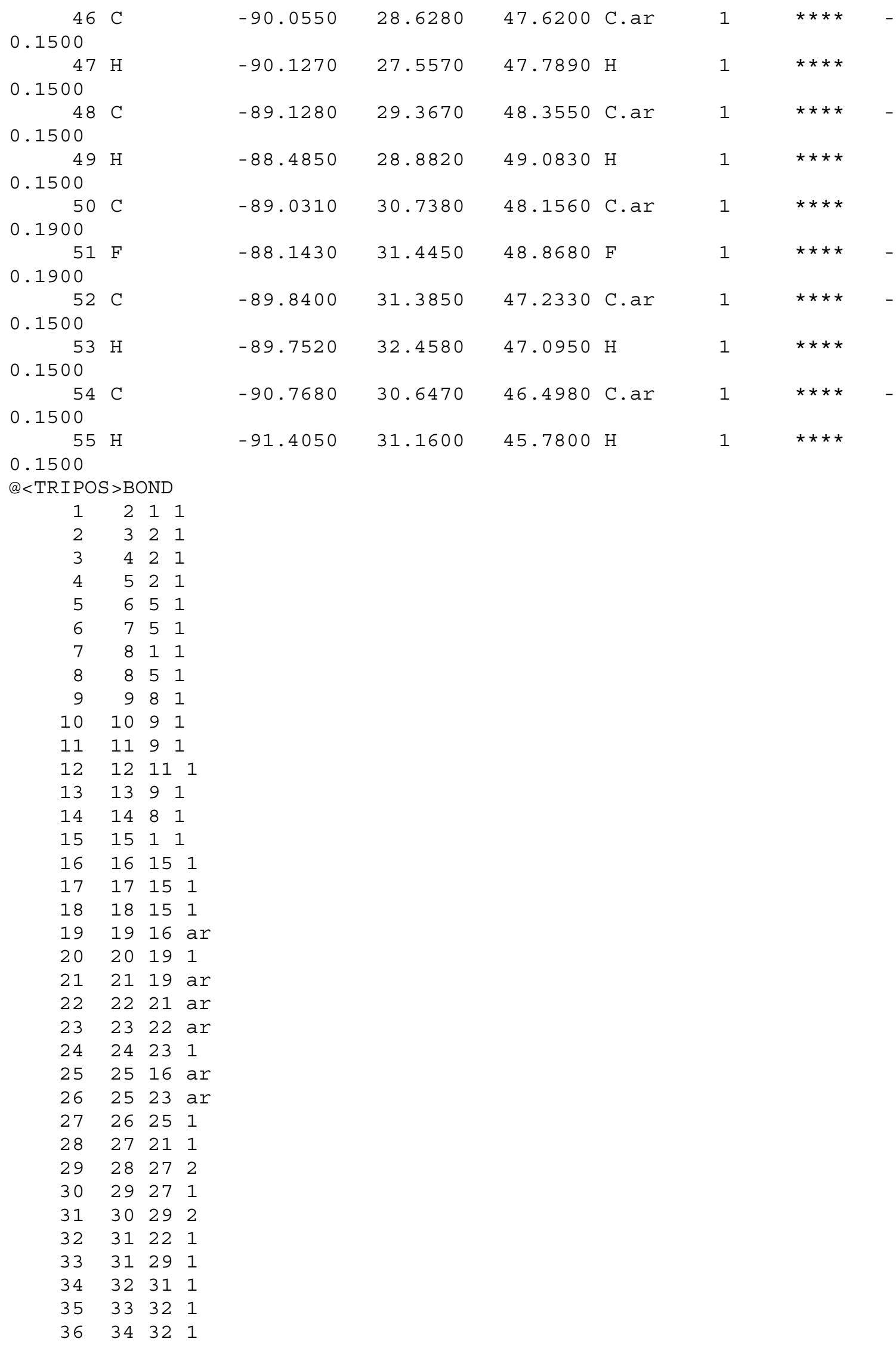




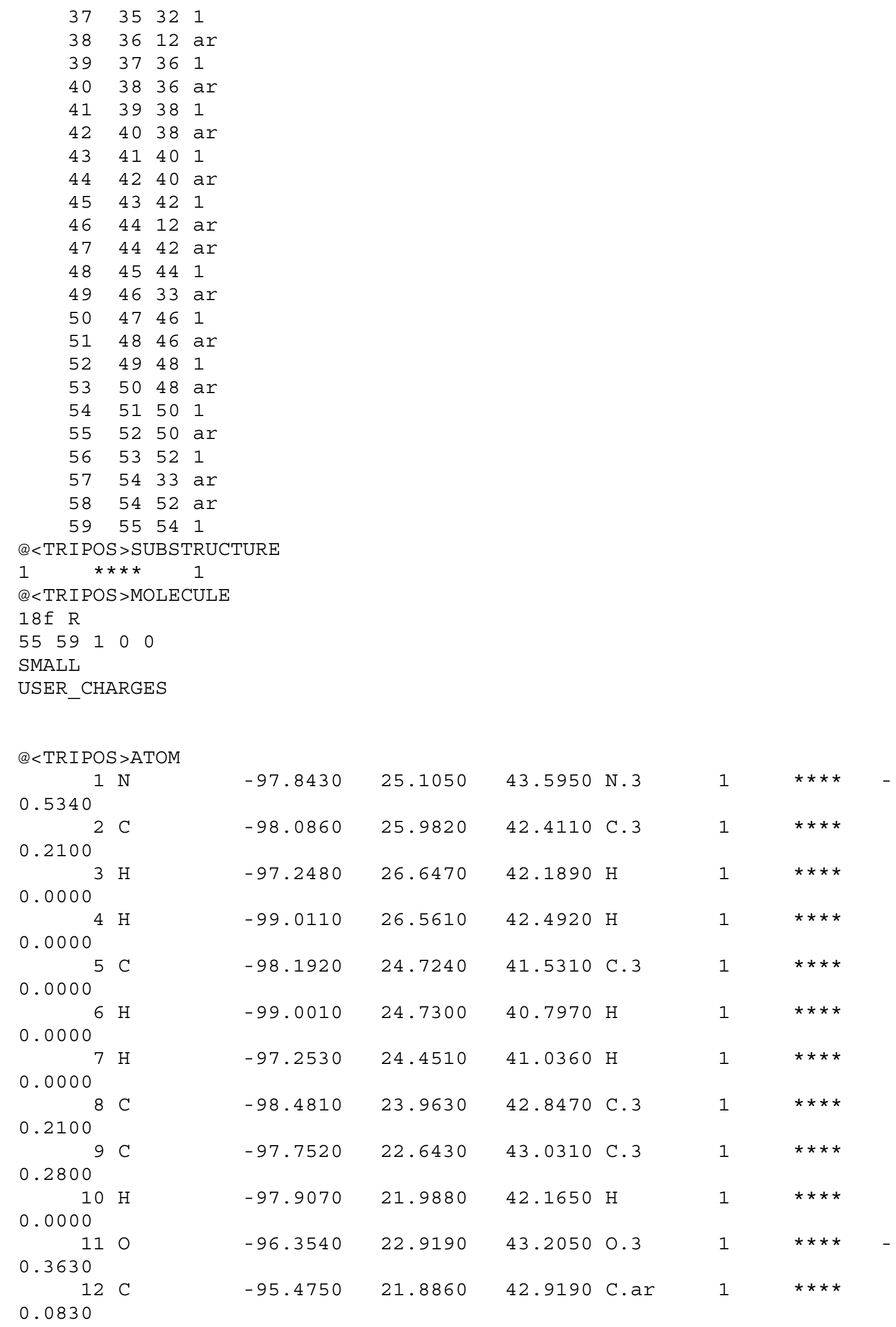




\begin{tabular}{|c|c|c|c|c|c|c|c|c|}
\hline 13 & $\mathrm{H}$ & -98.1360 & 22.1500 & 43.9330 & $\mathrm{H}$ & 1 & $\star \star \star \star$ & \\
\hline 0.0000 & & & & & & & & \\
\hline 14 & $\mathrm{H}$ & -99.5580 & 23.8890 & 43.0450 & $\mathrm{H}$ & 1 & $\star \star \star \star$ & \\
\hline 0.0000 & & & & & & & & \\
\hline 15 & $S$ & -98.0140 & 25.5250 & 45.2200 & S. 3 & 1 & $\star \star \star \star$ & \\
\hline 0.5380 & & & & & & & & \\
\hline 16 & $\mathrm{C}$ & -96.5320 & 26.4210 & 45.5520 & C.ar & 1 & 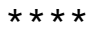 & \\
\hline 0.0070 & & & & & & & & \\
\hline 17 & O & -99.1320 & 26.4400 & 45.3330 & 0.3 & 1 & 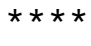 & \\
\hline 0.2150 & & & & & & & & \\
\hline 18 & O & -97.9800 & 24.3120 & 46.0110 & 0.3 & 1 & 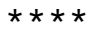 & \\
\hline 0.2150 & & & & & & & & \\
\hline 19 & C & -96.4270 & 27.1360 & 46.7470 & C.ar & 1 & $\star \star \star \star$ & \\
\hline 0.1500 & & & & & & & & \\
\hline 20 & $\mathrm{H}$ & -97.2430 & 27.1840 & 47.4640 & $\mathrm{H}$ & 1 & $\star \star \star \star$ & \\
\hline 0.1500 & & & & & & & & \\
\hline 21 & $\mathrm{C}$ & -95.2120 & 27.7560 & 47.0410 & C.ar & 1 & $\star \star \star \star$ & \\
\hline 0.0860 & & & & & & & & \\
\hline 22 & $\mathrm{C}$ & -94.1520 & 27.6970 & 46.1370 & C.ar & 1 & $\star \star \star \star$ & \\
\hline 0.1170 & & & & & & & & \\
\hline 23 & $\mathrm{C}$ & -94.2640 & 27.0280 & 44.9300 & C.ar & 1 & $\star \star \star \star ~$ & \\
\hline 0.1500 & & & & & & & & \\
\hline 24 & $\mathrm{H}$ & -93.4410 & 26.9730 & 44.2300 & $\mathrm{H}$ & 1 & $\star \star \star \star$ & \\
\hline 0.1500 & & & & & & & & \\
\hline 25 & $\mathrm{C}$ & -95.4710 & 26.3830 & 44.6360 & C.ar & 1 & $\star \star \star \star$ & \\
\hline 0.1500 & & & & & & & & \\
\hline 26 & $\mathrm{H}$ & -95.5630 & 25.8290 & 43.7030 & $\mathrm{H}$ & 1 & $\star \star \star \star$ & \\
\hline 0.1500 & & & & & & & & \\
\hline 27 & $\mathrm{C}$ & -94.7550 & 28.4910 & 48.2120 & C. 2 & 1 & $\star \star \star \star *$ & \\
\hline 0.4840 & & & & & & & & \\
\hline 28 & $\mathrm{O}$ & -95.3910 & 28.7530 & 49.2160 & 0.2 & 1 & 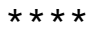 & \\
\hline 0.5700 & & & & & & & & \\
\hline 29 & $\mathrm{C}$ & -93.3220 & 28.8700 & 47.9550 & C. 2 & 1 & 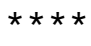 & \\
\hline 0.6300 & & & & & & & & \\
\hline 30 & O & -92.5250 & 29.4820 & 48.6610 & 0.2 & 1 & $\star \star \star \star *$ & \\
\hline 0.5700 & & & & & & & & \\
\hline $\begin{array}{r}31 \\
0.4770\end{array}$ & $\mathrm{~N}$ & -93.0420 & 28.3690 & 46.6690 & N.am & 1 & $\star \star \star * *$ & \\
\hline 32 & $\mathrm{C}$ & -91.7670 & 28.5250 & 46.0100 & C. 3 & 1 & $\star \star \star *$ & \\
\hline 0.4440 & & & & & & & & \\
\hline $\begin{array}{r}33 \\
0.1440^{3}\end{array}$ & $\mathrm{C}$ & -90.7780 & 29.3610 & 46.7810 & C.ar & 1 & 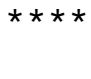 & \\
\hline $0.0000^{34}$ & $\mathrm{H}$ & -91.3220 & 27.5370 & 45.8420 & $\mathrm{H}$ & 1 & $\star \star \star \star$ & \\
\hline 35 & $\mathrm{H}$ & -91.9510 & 28.9440 & 45.0130 & $\mathrm{H}$ & 1 & 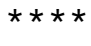 & \\
\hline 0.0000 & & & & & & & & \\
\hline 36 & $\mathrm{C}$ & -95.7850 & 20.5190 & 42.9290 & C.ar & 1 & $\star \star \star \star$ & \\
\hline 0.1500 & & & & & & & & \\
\hline 37 & $\mathrm{H}$ & -96.7790 & 20.1610 & 43.1660 & $\mathrm{H}$ & 1 & $\star \star \star \star$ & \\
\hline 0.1500 & & & & & & & & \\
\hline 38 & $\mathrm{C}$ & -94.7980 & 19.5790 & 42.6230 & C.ar & 1 & $\star \star \star \star \star$ & \\
\hline 0.1500 & & & & & & & & \\
\hline 39 & $\mathrm{H}$ & -95.0410 & 18.5200 & 42.6290 & $\mathrm{H}$ & 1 & $\star \star \star \star$ & \\
\hline 0.1500 & & & & & & & & \\
\hline 40 & $\mathrm{C}$ & -93.5050 & 19.9940 & 42.3080 & C.ar & 1 & $\star * * *$ & \\
\hline 0.1500 & 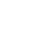 & & & & & & & \\
\hline
\end{tabular}




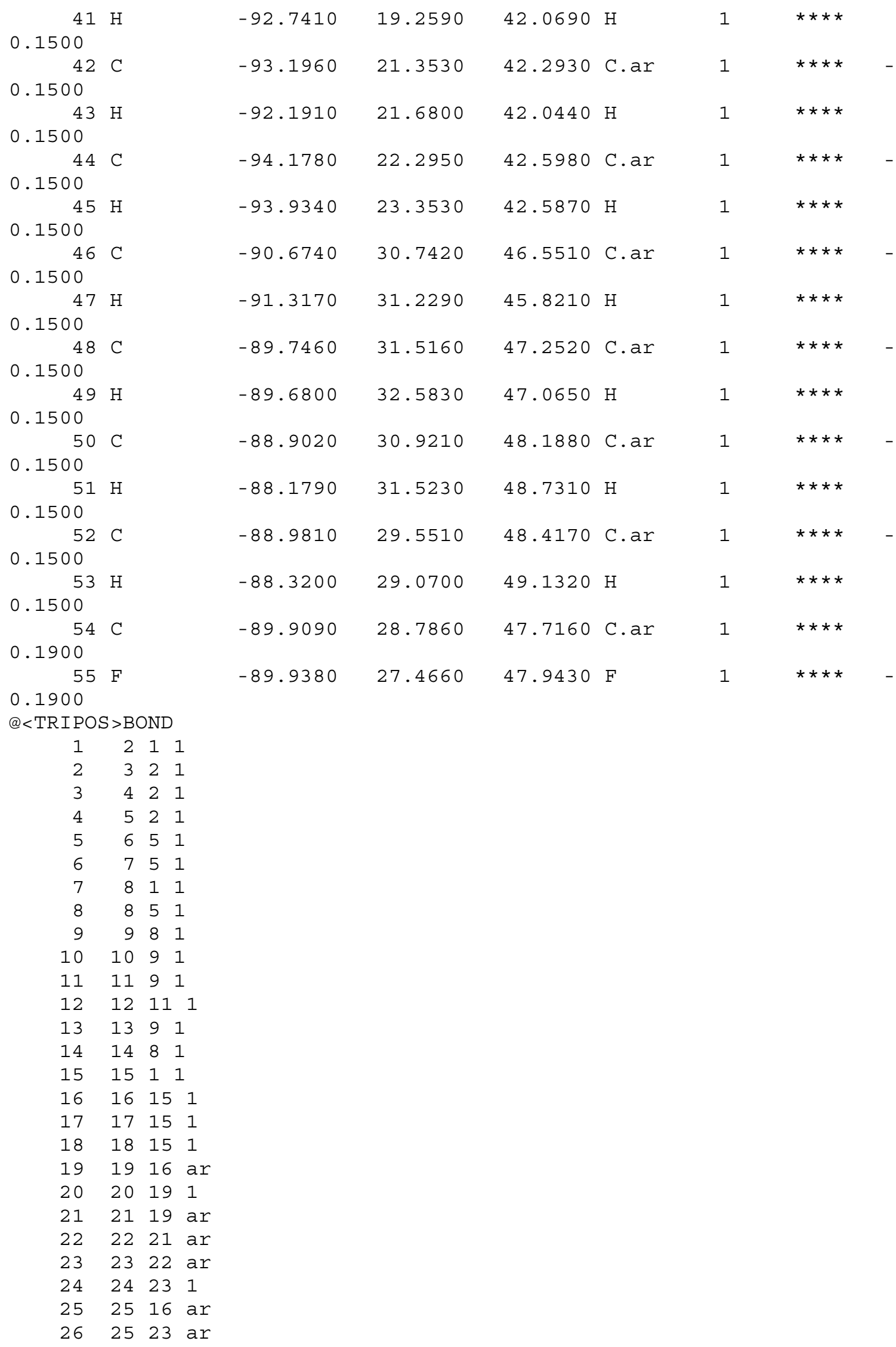




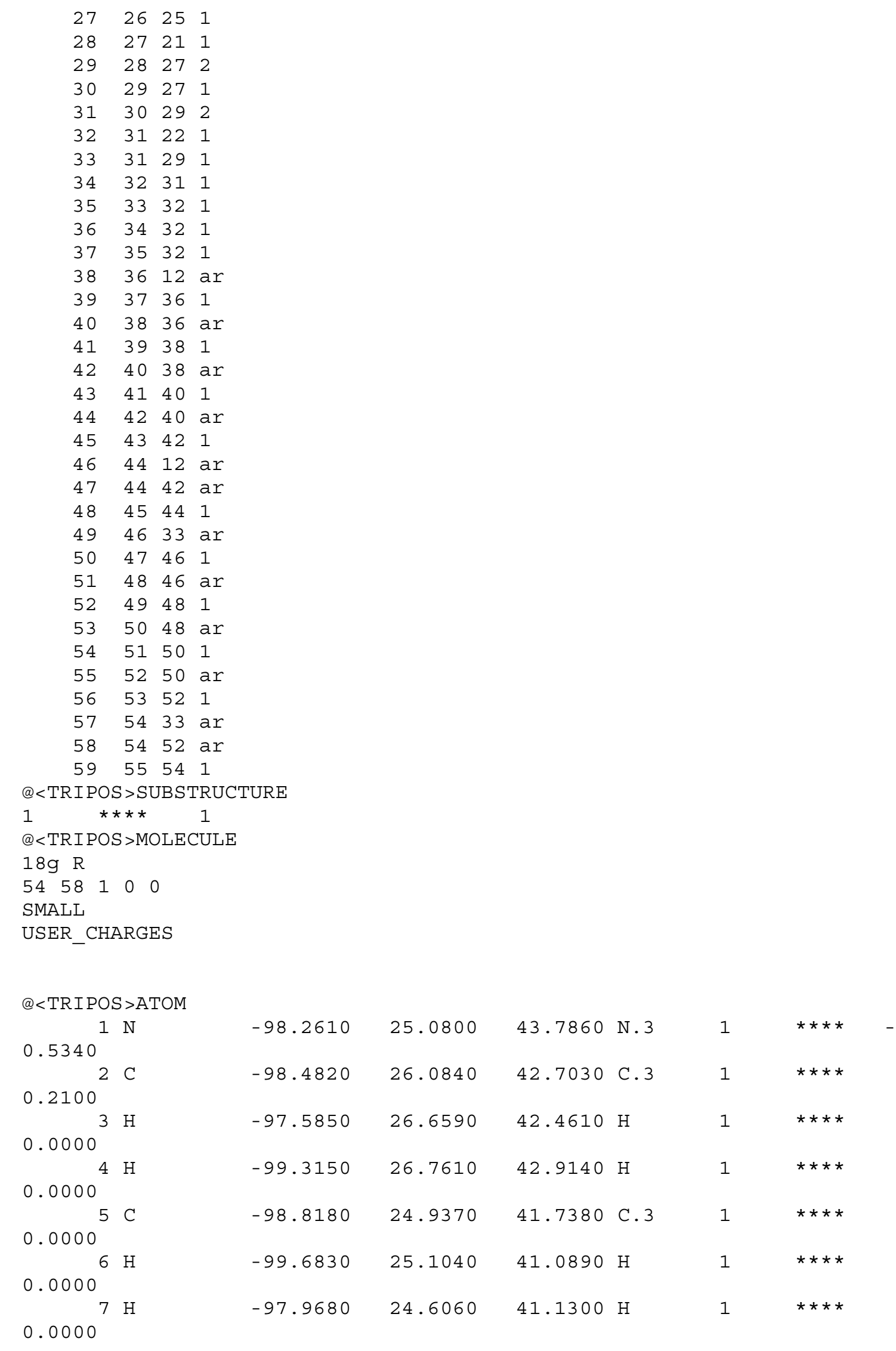




\begin{tabular}{|c|c|c|c|c|c|c|c|}
\hline 8 & $\mathrm{C}$ & -99.0820 & 24.0900 & 43.0060 & C. 3 & 1 & $\star \star \star \star \star$ \\
\hline \multicolumn{8}{|c|}{0.2100} \\
\hline 9 & $\mathrm{C}$ & -98.4940 & 22.6890 & 43.0050 & C. 3 & 1 & $\star \star \star \star$ \\
\hline \multicolumn{8}{|c|}{0.2800} \\
\hline 10 & $\mathrm{H}$ & -98.6030 & 22.2210 & 42.0190 & $\mathrm{H}$ & 1 & $\star \star \star \star *$ \\
\hline \multicolumn{8}{|c|}{0.0000} \\
\hline 11 & O & -97.1080 & 22.7860 & 43.3620 & 0.3 & 1 & 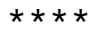 \\
\hline \multicolumn{8}{|c|}{0.3630} \\
\hline 12 & $\mathrm{C}$ & -96.3040 & 21.6990 & 43.0540 & C.ar & 1 & $\star \star \star \star *$ \\
\hline \multicolumn{8}{|c|}{0.0830} \\
\hline 13 & $\mathrm{H}$ & -99.0190 & 22.0840 & 43.7560 & $\mathrm{H}$ & 1 & $\star \star \star \star$ \\
\hline \multicolumn{8}{|c|}{0.0000} \\
\hline 14 & $\mathrm{H}$ & -100.1390 & 24.1120 & 43.2990 & $\mathrm{H}$ & 1 & $\star \star \star \star$ \\
\hline \multicolumn{8}{|c|}{0.0000} \\
\hline 15 & $S$ & -98.2310 & 25.3600 & 45.4470 & S. 3 & 1 & 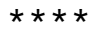 \\
\hline \multicolumn{8}{|c|}{0.5380} \\
\hline 16 & $\mathrm{C}$ & -96.7330 & 26.2610 & 45.6760 & C.ar & 1 & $\star \star \star \star$ \\
\hline \multicolumn{8}{|c|}{$\begin{array}{r}0.0070 \\
170\end{array}$} \\
\hline \multicolumn{8}{|c|}{0.2150} \\
\hline \multicolumn{7}{|c|}{0.2150} & $\star * \star *$ \\
\hline \multicolumn{8}{|c|}{0.1500} \\
\hline \multicolumn{5}{|c|}{0.1500} & $\mathrm{H}$ & & $\star \star \star \star$ \\
\hline \multicolumn{7}{|c|}{0.0860} & $\star \star \star \star$ \\
\hline \multicolumn{7}{|c|}{0.1170} & $\star \star \star \star$ \\
\hline \multicolumn{8}{|c|}{0.1500} \\
\hline \multicolumn{7}{|c|}{0.1500} & 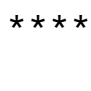 \\
\hline \multicolumn{7}{|c|}{0.1500} & $\star \star \star \star *$ \\
\hline \multicolumn{8}{|c|}{0.1500} \\
\hline \multicolumn{7}{|c|}{0.4840} & $\star \star \star \star *$ \\
\hline \multicolumn{7}{|c|}{0.5700} & $\star \star \star \star$ \\
\hline \multicolumn{8}{|c|}{0.6300} \\
\hline \multicolumn{8}{|c|}{0.5700} \\
\hline \multicolumn{7}{|c|}{0.4770} & $\star \star \star \star$ \\
\hline \multicolumn{7}{|c|}{0.4440} & $\star \star \star \star$ \\
\hline \multicolumn{8}{|c|}{0.1440} \\
\hline $\begin{array}{r}34 \\
0.0000^{34}\end{array}$ & $\mathrm{H}$ & -91.5450 & 27.3600 & 45.6990 & $\mathrm{H}$ & 1 & $\star \star \star * *$ \\
\hline $0.0000^{35}$ & $\mathrm{H}$ & -92.1040 & 28.7760 & 44.8450 & $\mathrm{H}$ & 1 & $\star \star \star \star$ \\
\hline
\end{tabular}




\begin{tabular}{|c|c|c|c|c|c|c|c|c|c|}
\hline 36 & $C$ & & -96.7460 & 20.3870 & 42.8360 & C.ar & 1 & $\star \star \star \star$ & - \\
\hline \multicolumn{10}{|c|}{0.1500} \\
\hline 37 & \multicolumn{2}{|c|}{$7 \mathrm{H}$} & -97.7950 & 20.1210 & 42.8900 & $\mathrm{H}$ & 1 & $\star \star \star \star$ & \\
\hline \multicolumn{9}{|c|}{0.1500} & \\
\hline 38 & \multicolumn{2}{|l|}{$\mathrm{C}$} & -95.8220 & 19.3840 & 42.5370 & C.ar & 1 & $\star \star \star \star$ & 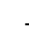 \\
\hline \multicolumn{9}{|c|}{0.1500} & \\
\hline 39 & \multicolumn{2}{|l|}{$\mathrm{H}$} & -96.1650 & 18.3670 & 42.3650 & $\mathrm{H}$ & 1 & $\star \star \star \star$ & \\
\hline \multicolumn{9}{|c|}{0.1500} & \\
\hline 40 & \multicolumn{2}{|l|}{$\mathrm{C}$} & -94.4620 & 19.6800 & 42.4540 & C.ar & 1 & $\star \star \star \star$ & 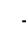 \\
\hline \multicolumn{9}{|c|}{0.1500} & \\
\hline 41 & \multicolumn{2}{|l|}{$\mathrm{H}$} & -93.7460 & 18.8950 & 42.2230 & $\mathrm{H}$ & 1 & $\star \star \star \star$ & \\
\hline \multicolumn{9}{|c|}{0.1500} & \\
\hline 42 & \multicolumn{2}{|l|}{$\mathrm{C}$} & -94.0210 & 20.9850 & 42.6670 & C.ar & 1 & $\star \star \star \star$ & 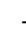 \\
\hline \multicolumn{9}{|l|}{0.1500} & \\
\hline 43 & \multicolumn{2}{|l|}{$\mathrm{H}$} & -92.9640 & 21.2230 & 42.6020 & $\mathrm{H}$ & 1 & $\star \star \star \star$ & \\
\hline \multicolumn{9}{|l|}{0.1500} & \\
\hline 44 & \multicolumn{2}{|l|}{$\mathrm{C}$} & -94.9420 & 21.9900 & 42.9650 & C.ar & 1 & $\star \star \star \star$ & 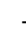 \\
\hline 0.1500 & & & & & & & & & \\
\hline 45 & $\mathrm{H}$ & & -94.5960 & 23.0060 & 43.1310 & $\mathrm{H}$ & 1 & $\star \star \star \star$ & \\
\hline 0.1500 & & & & & & & & & \\
\hline 46 & C & & -90.0330 & 28.5850 & 47.5070 & C.ar & 1 & $\star \star \star \star$ & \\
\hline 0.1600 & & & & & & & & & \\
\hline 47 & $\mathrm{H}$ & & -90.0600 & 27.5160 & 47.6970 & $\mathrm{H}$ & 1 & $\star \star \star \star$ & \\
\hline 0.1500 & & & & & & & & & \\
\hline 48 & $\mathrm{~N}$ & & -89.1080 & 29.2600 & 48.2310 & N.ar & 1 & $\star \star \star \star$ & \\
\hline 0.6200 & & & & & & & & & \\
\hline 49 & C & & -89.0400 & 30.5940 & 48.0270 & C.ar & 1 & $\star \star \star \star$ & \\
\hline 0.5000 & & & & & & & & & \\
\hline 50 & $F$ & & -88.1350 & 31.2830 & 48.7390 & $\mathrm{~F}$ & 1 & $\star \star \star \star$ & \\
\hline 0.1900 & & & & & & & & & \\
\hline 51 & C & & -89.8450 & 31.2830 & 47.1360 & C.ar & 1 & 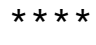 & \\
\hline 0.1500 & & & & & & & & & \\
\hline 52 & $\mathrm{H}$ & & -89.7360 & 32.3540 & 47.0140 & $\mathrm{H}$ & 1 & $\star \star \star \star$ & \\
\hline 0.1500 & & & & & & & & & \\
\hline 53 & C & & -90.7920 & 30.5640 & 46.4120 & C.ar & 1 & $\star \star \star \star ~$ & \\
\hline 0.1500 & & & & & & & & & \\
\hline 54 & $\mathrm{H}$ & & -91.4370 & 31.0860 & 45.7070 & $\mathrm{H}$ & 1 & $\star \star \star \star$ & \\
\hline 0.1500 & & & & & & & & & \\
\hline @ < TRIPO & $S>E$ & BOND & & & & & & & \\
\hline 1 & 2 & 11 & & & & & & & \\
\hline 2 & 3 & 21 & & & & & & & \\
\hline 3 & 4 & 21 & & & & & & & \\
\hline 4 & 5 & 21 & & & & & & & \\
\hline 5 & 6 & 51 & & & & & & & \\
\hline 6 & 7 & 51 & & & & & & & \\
\hline 7 & 8 & 11 & & & & & & & \\
\hline 8 & 8 & 51 & & & & & & & \\
\hline 9 & 9 & 81 & & & & & & & \\
\hline 10 & 10 & 91 & & & & & & & \\
\hline 11 & 11 & 91 & & & & & & & \\
\hline 12 & 12 & 111 & & & & & & & \\
\hline 13 & 13 & 91 & & & & & & & \\
\hline 14 & 14 & 81 & & & & & & & \\
\hline 15 & 15 & 11 & & & & & & & \\
\hline 16 & 16 & 151 & & & & & & & \\
\hline 17 & 17 & 151 & & & & & & & \\
\hline 18 & 18 & 151 & & & & & & & \\
\hline
\end{tabular}




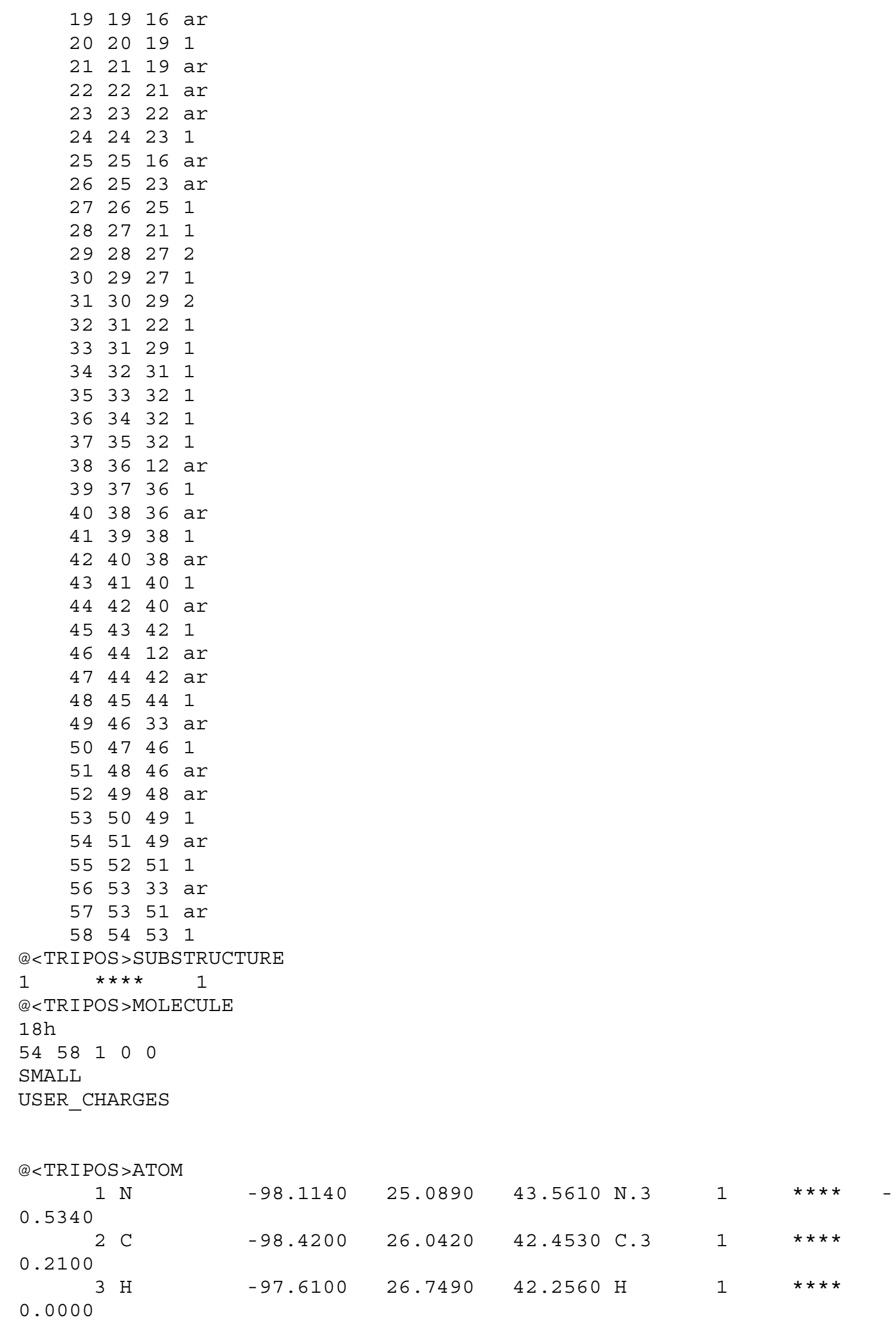




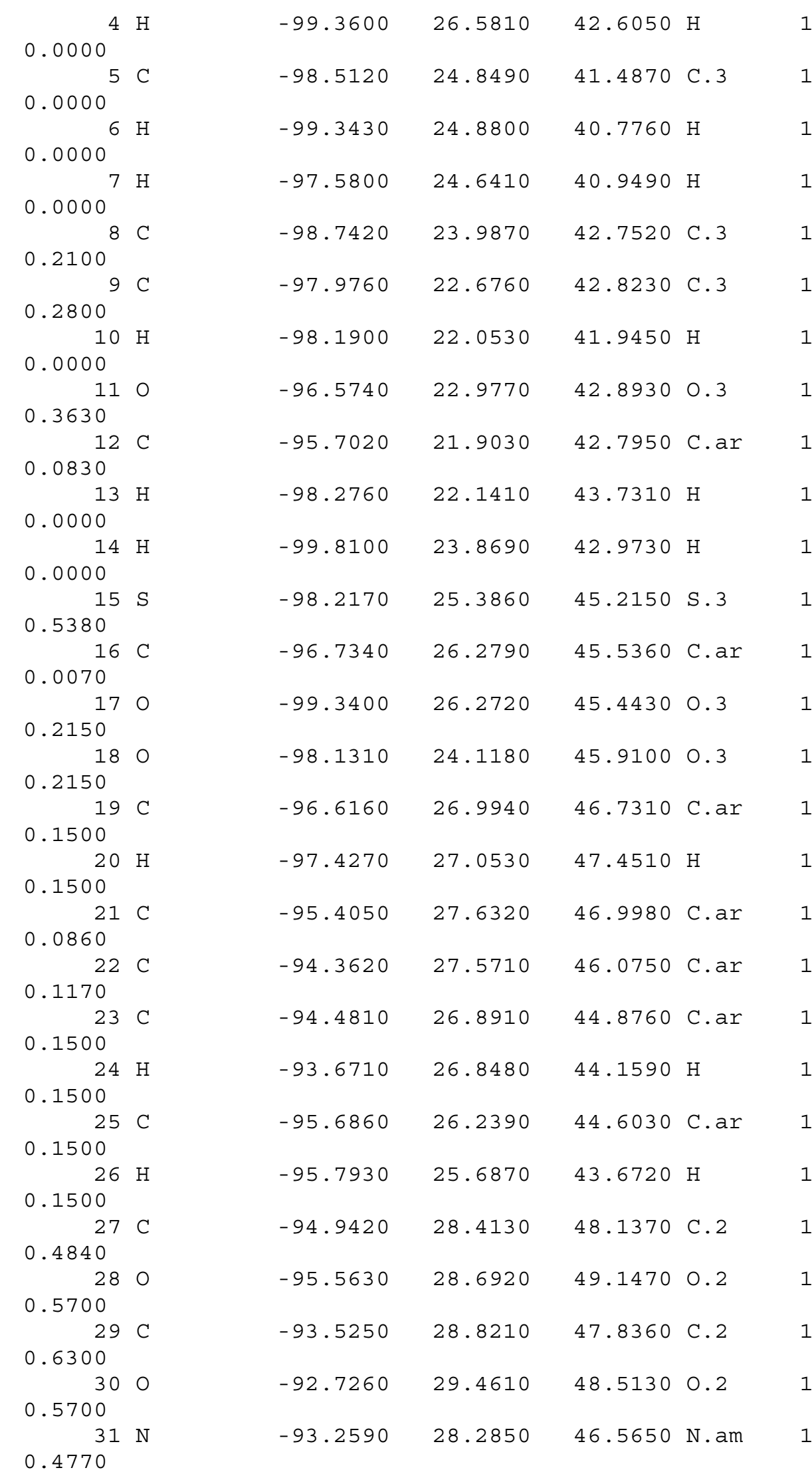




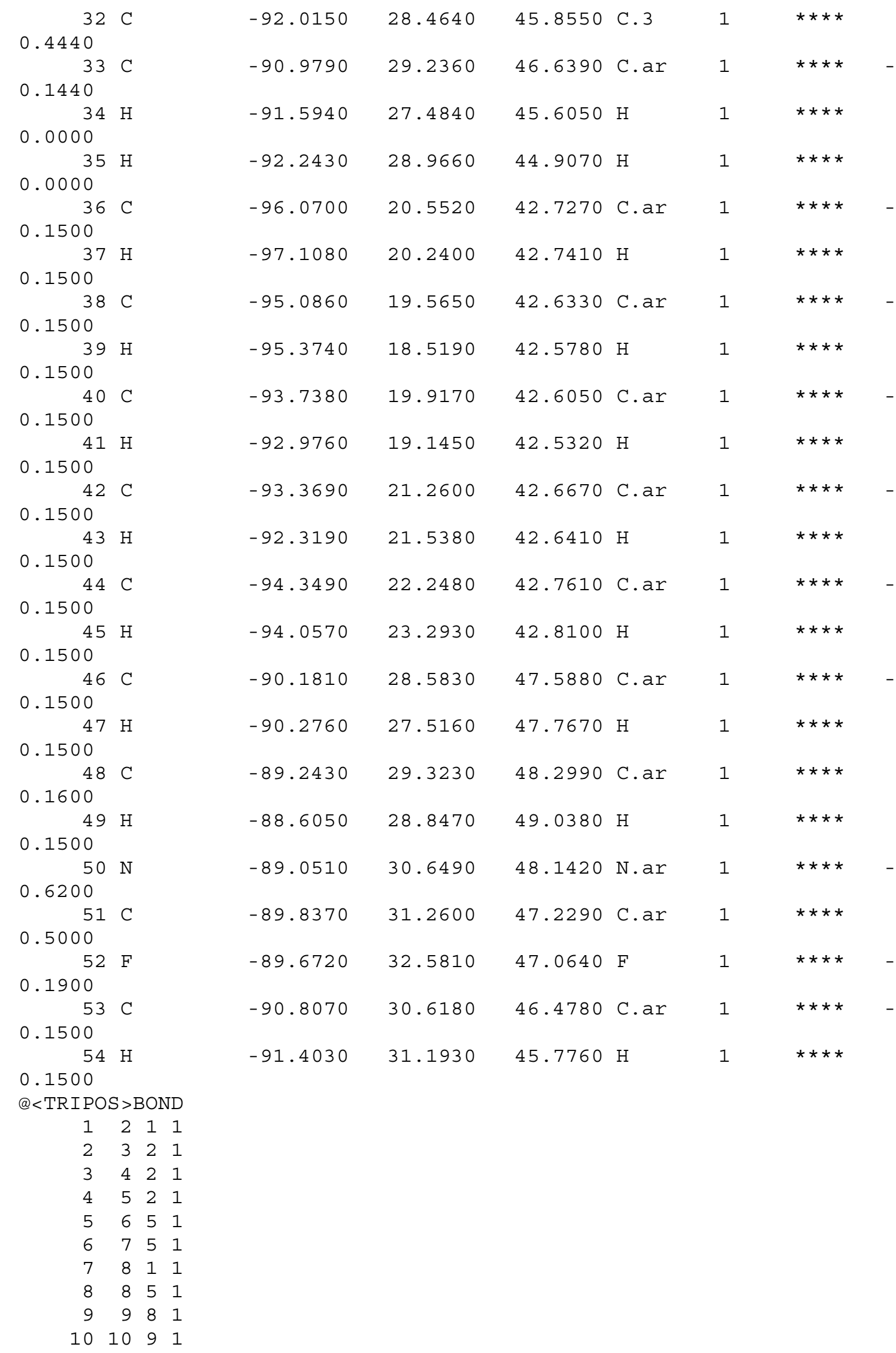




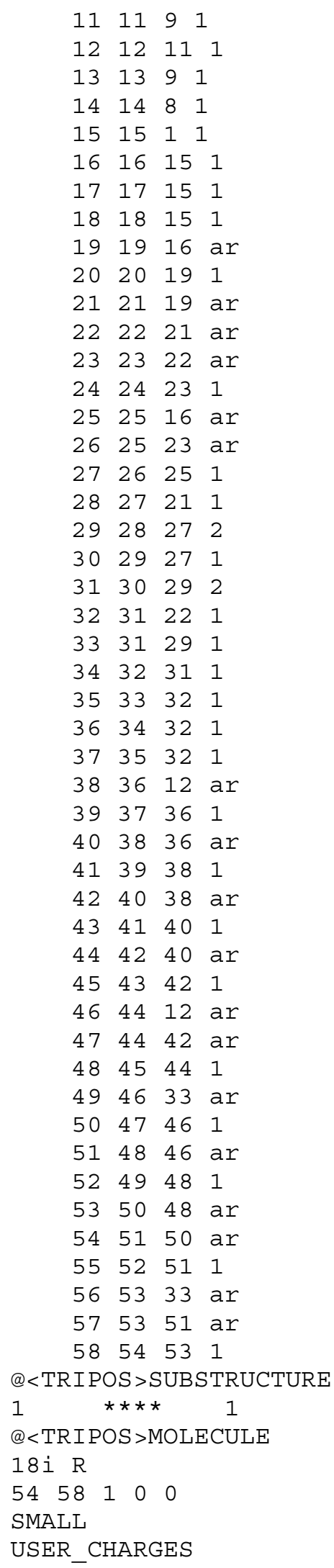




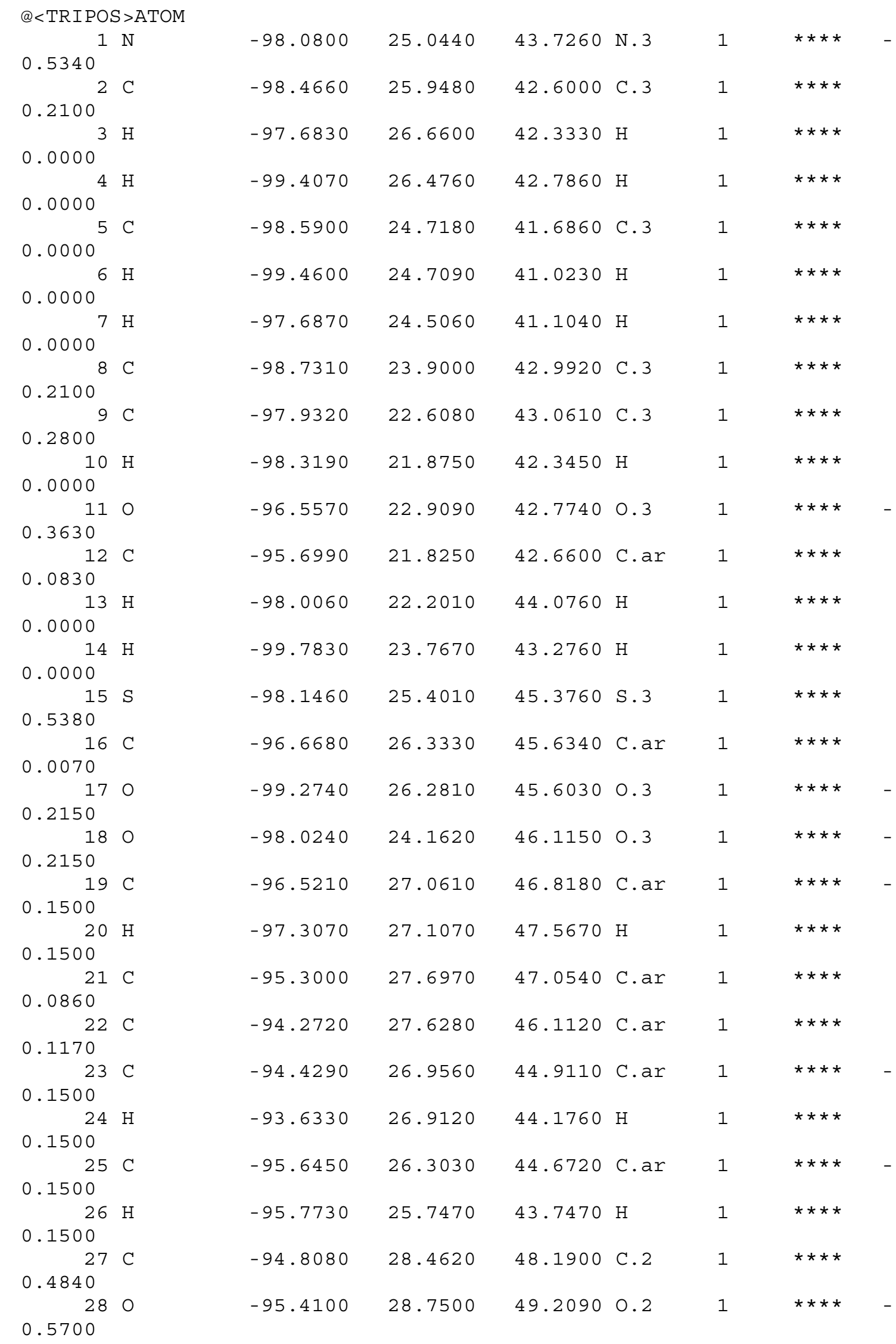




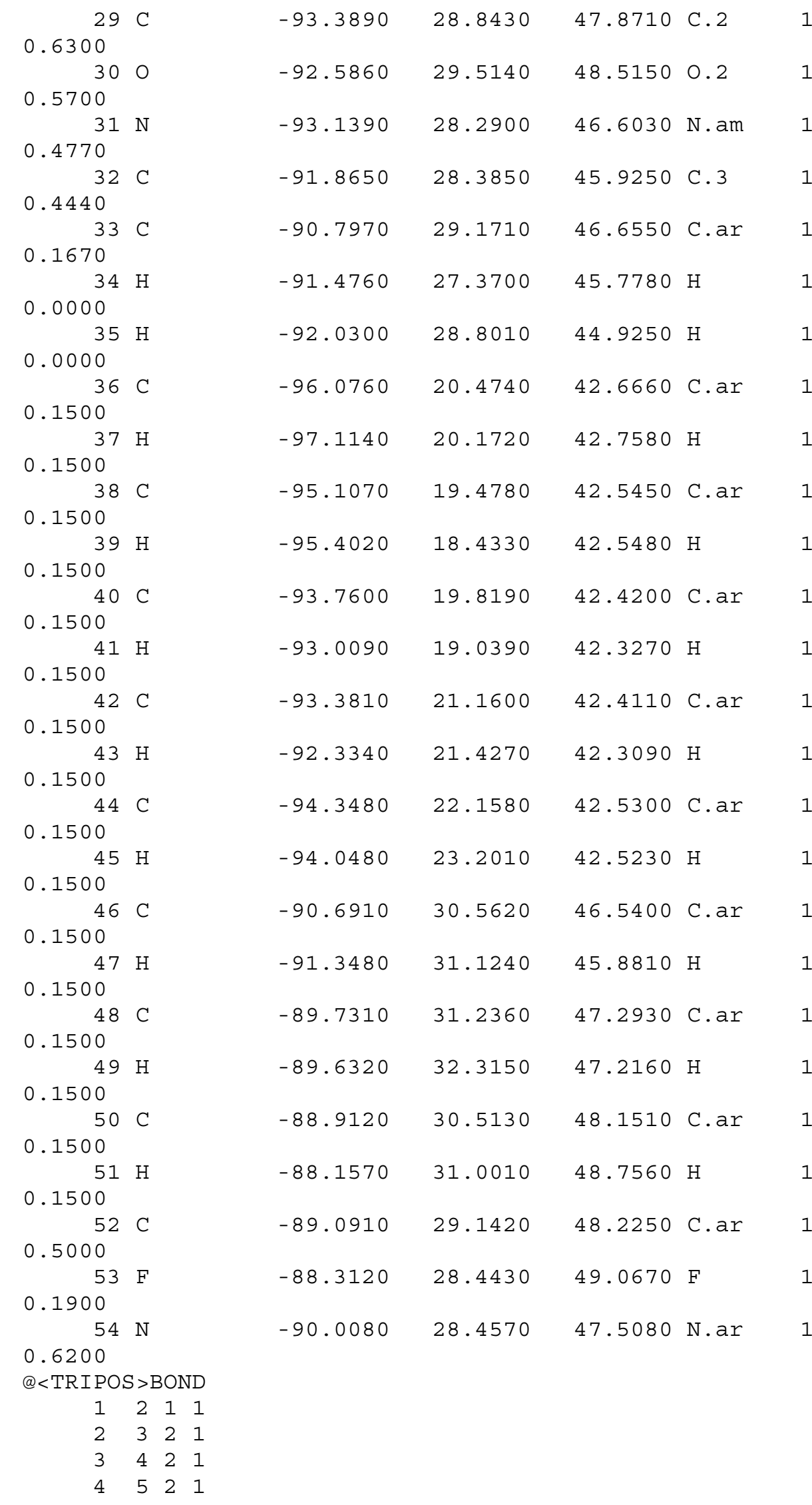




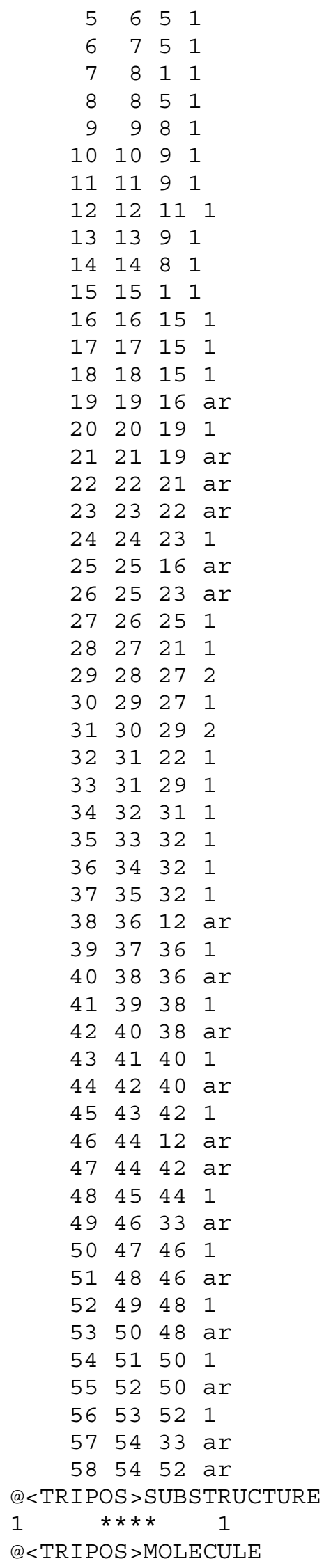




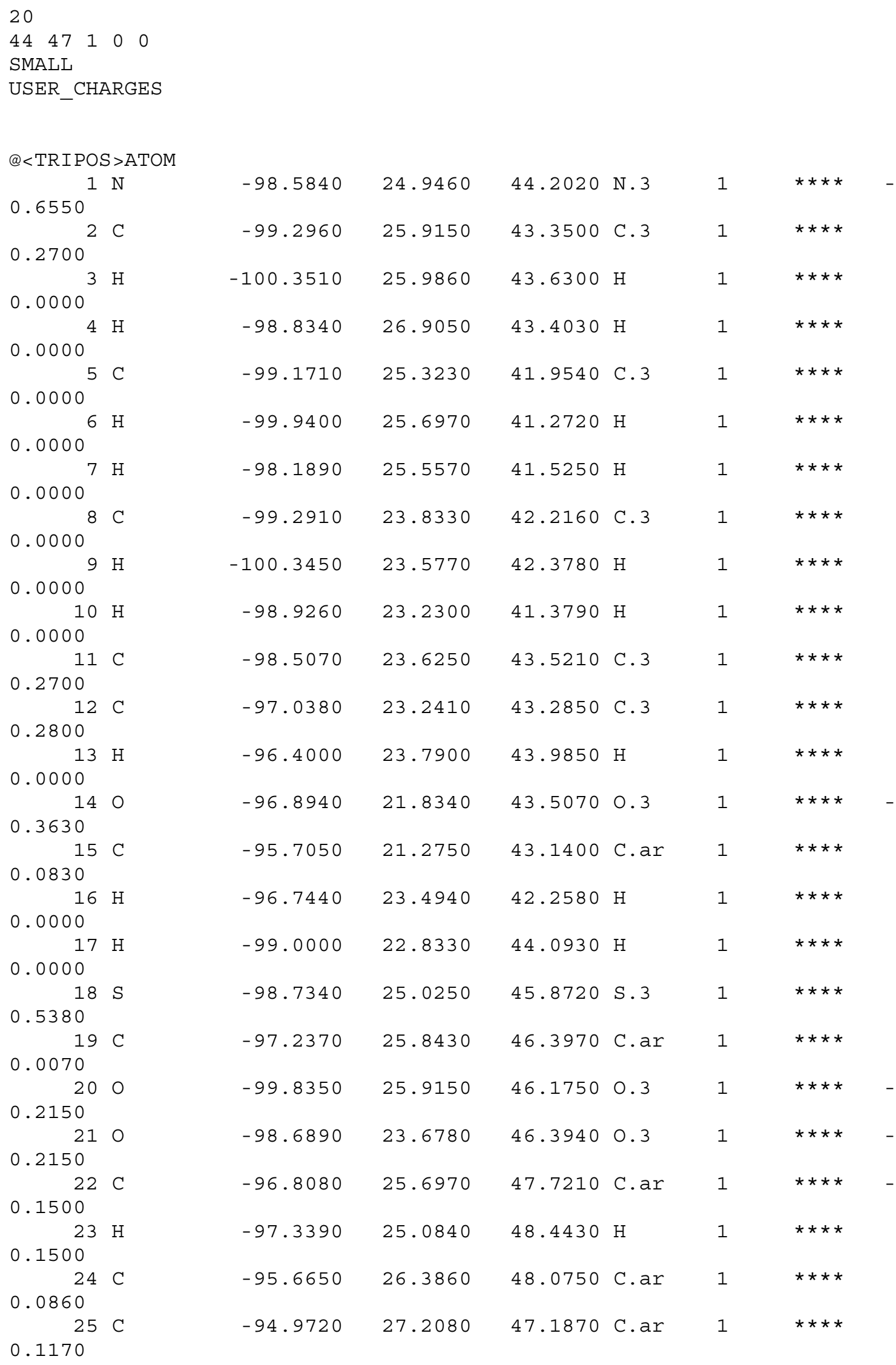




\begin{tabular}{|c|c|c|c|c|c|c|c|c|c|}
\hline 26 & C & & -95.4010 & 27.3810 & 45.8870 & C.ar & 1 & $\star \star \star \star$ & - \\
\hline \multicolumn{9}{|c|}{0.1500} & \\
\hline 27 & $\mathrm{H}$ & & -94.8780 & 28.0300 & 45.1930 & $\mathrm{H}$ & 1 & $\star \star \star \star$ & \\
\hline \multicolumn{9}{|c|}{0.1500} & \\
\hline 28 & $\mathrm{C}$ & & -96.5550 & 26.6910 & 45.4930 & C.ar & 1 & $\star \star \star \star$ & 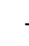 \\
\hline \multicolumn{9}{|c|}{0.1500} & \\
\hline 29 & $\mathrm{H}$ & & -96.9300 & 26.8230 & 44.4780 & $\mathrm{H}$ & 1 & 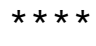 & \\
\hline \multicolumn{9}{|c|}{0.1500} & \\
\hline 30 & $\mathrm{C}$ & & -94.9340 & 26.4670 & 49.3360 & C. 2 & 1 & $\star \star \star \star$ & \\
\hline \multicolumn{9}{|c|}{0.4840} & \\
\hline 31 & 0 & & -95.2090 & 25.9220 & 50.3910 & 0.2 & 1 & $\star \star \star \star$ & 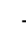 \\
\hline \multicolumn{9}{|c|}{0.5700} & \\
\hline 32 & - $\mathrm{C}$ & & -93.7480 & 27.3900 & 49.1090 & C. 2 & 1 & $\star \star \star \star$ & \\
\hline \multicolumn{9}{|c|}{0.6300} & \\
\hline 33 & 0 & & -92.9030 & 27.7040 & 49.9160 & 0.2 & 1 & $\star \star \star \star$ & 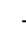 \\
\hline \multicolumn{9}{|c|}{0.5700} & \\
\hline 34 & $\mathrm{~N}$ & & -93.8630 & 27.7900 & 47.7920 & N. am & 1 & $\star \star \star \star$ & 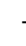 \\
\hline \multicolumn{9}{|c|}{0.5470} & \\
\hline 35 & $\mathrm{H}$ & & -93.2100 & 28.4260 & 47.3570 & $\mathrm{H}$ & 1 & $\star \star \star \star$ & \\
\hline \multicolumn{9}{|c|}{0.3700} & \\
\hline 36 & $\mathrm{C}$ & & -95.7630 & 19.9330 & 42.7690 & C.ar & 1 & $\star \star \star \star$ & 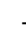 \\
\hline \multicolumn{9}{|c|}{0.1500} & \\
\hline 37 & $\mathrm{H}$ & & -96.7110 & 19.4040 & 42.7870 & $\mathrm{H}$ & 1 & $\star \star \star \star$ & \\
\hline \multicolumn{9}{|c|}{0.1500} & \\
\hline 38 & $\mathrm{C}$ & & -94.6040 & 19.2690 & 42.3740 & C.ar & 1 & $\star \star \star \star$ & \\
\hline \multicolumn{9}{|c|}{0.1500} & \\
\hline 39 & $\mathrm{H}$ & & -94.6300 & 18.2250 & 42.0820 & $\mathrm{H}$ & 1 & $\star \star \star \star$ & \\
\hline \multicolumn{9}{|c|}{0.1500} & \\
\hline 40 & $\mathrm{C}$ & & -93.4150 & 19.9780 & 42.3630 & C.ar & 1 & $\star \star \star \star$ & \\
\hline \multicolumn{9}{|c|}{0.1600} & \\
\hline 41 & - $\mathrm{H}$ & & -92.4830 & 19.5060 & 42.0620 & $\mathrm{H}$ & 1 & 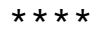 & \\
\hline \multicolumn{9}{|c|}{0.1500} & \\
\hline 42 & $\mathrm{~N}$ & & -93.3150 & 21.2760 & 42.7160 & N.ar & 1 & $\star \star \star \star$ & \\
\hline 0.6200 & & & & & & & & & \\
\hline 43 & C & & -94.4610 & 21.8930 & 43.0990 & C.ar & 1 & $\star \star \star \star ~$ & \\
\hline 0.1600 & & & & & & & & & \\
\hline 44 & $\mathrm{H}$ & & -94.3170 & 22.9320 & 43.3780 & $\mathrm{H}$ & 1 & $\star \star \star \star$ & \\
\hline 0.1500 & & & & & & & & & \\
\hline @ < TRIPO & $S>$ & BOND & & & & & & & \\
\hline 1 & 2 & 11 & & & & & & & \\
\hline 2 & 3 & 21 & & & & & & & \\
\hline 3 & 4 & 21 & & & & & & & \\
\hline 4 & 5 & 21 & & & & & & & \\
\hline 5 & 6 & 51 & & & & & & & \\
\hline 6 & 7 & 51 & & & & & & & \\
\hline 7 & 8 & 51 & & & & & & & \\
\hline 8 & 9 & 81 & & & & & & & \\
\hline 9 & 10 & 81 & & & & & & & \\
\hline 10 & 11 & 11 & & & & & & & \\
\hline 11 & 11 & 81 & & & & & & & \\
\hline 12 & 12 & 111 & & & & & & & \\
\hline 13 & 13 & 121 & & & & & & & \\
\hline 14 & 14 & 121 & & & & & & & \\
\hline 15 & 15 & 141 & & & & & & & \\
\hline 16 & 16 & 121 & & & & & & & \\
\hline 17 & 17 & 111 & & & & & & & \\
\hline 18 & 18 & 11 & & & & & & & \\
\hline
\end{tabular}




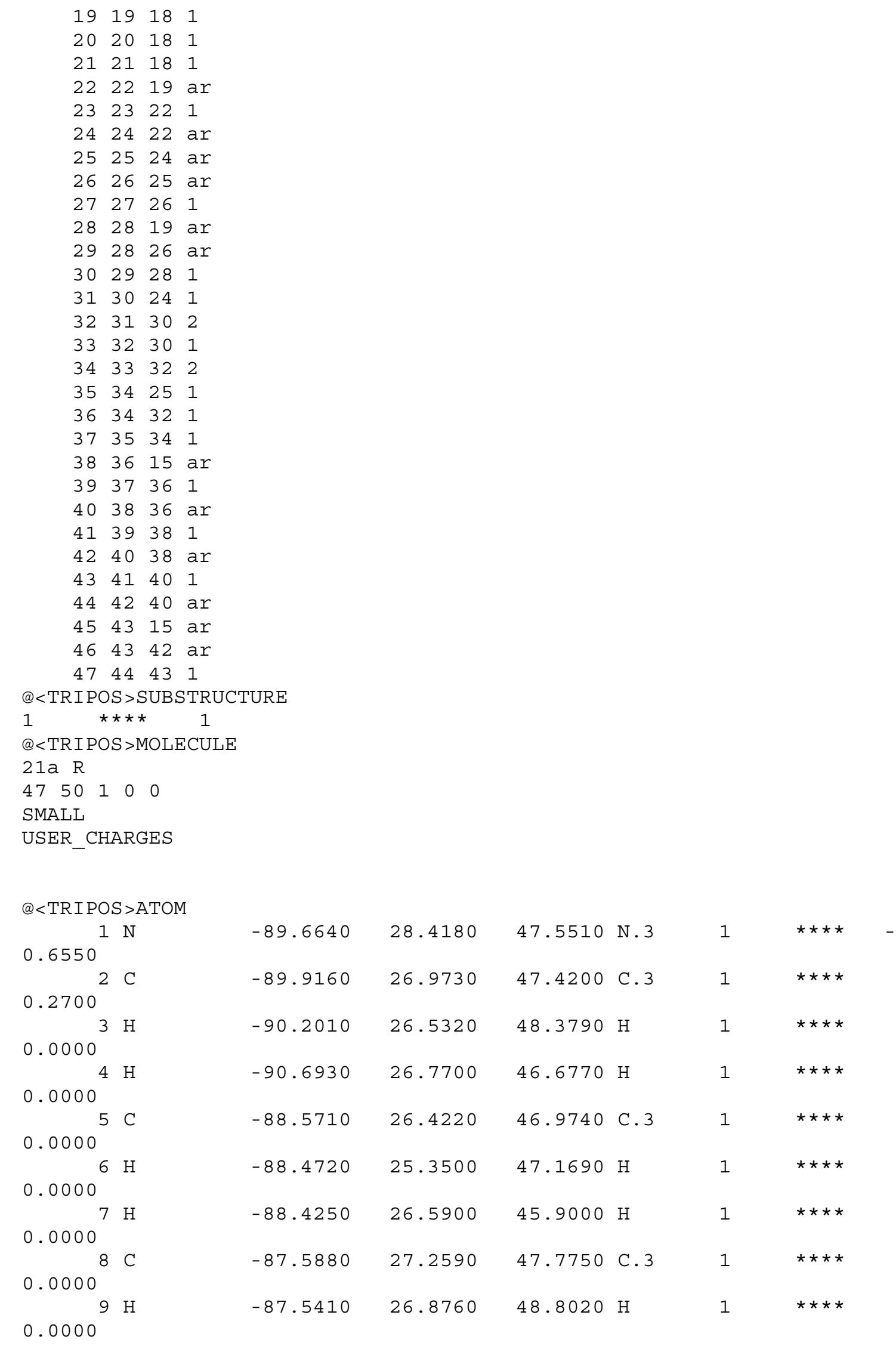




\begin{tabular}{|c|c|c|c|c|c|}
\hline \multirow{2}{*}{$0.0000^{10}$} & \multirow{2}{*}{$\mathrm{H}$} & \multirow[t]{2}{*}{-86.5760} & \multirow[t]{2}{*}{27.2290} & \multirow[t]{2}{*}{47.3610} & \multirow[t]{2}{*}{$\mathrm{H}$} \\
\hline & & & & & \\
\hline 11 & $\mathrm{C}$ & -88.2150 & 28.6620 & 47.7810 & C. 3 \\
\hline \multicolumn{6}{|c|}{0.2700} \\
\hline 12 & $\mathrm{C}$ & -87.6480 & 29.5750 & 46.6800 & C. 3 \\
\hline \multicolumn{6}{|c|}{0.2800} \\
\hline 13 & $\mathrm{H}$ & -88.2100 & 30.5080 & 46.5640 & $\mathrm{H}$ \\
\hline \multicolumn{6}{|c|}{0.0000} \\
\hline 14 & O & -86.2520 & 29.8320 & 46.8770 & 0.3 \\
\hline \multicolumn{6}{|c|}{0.3630} \\
\hline 15 & $\mathrm{C}$ & -85.6720 & 30.8190 & 46.1350 & C.ar \\
\hline \multicolumn{6}{|c|}{0.0830} \\
\hline 16 & $\mathrm{H}$ & -87.7340 & 29.0710 & 45.7100 & $\mathrm{H}$ \\
\hline \multicolumn{6}{|c|}{0.0000} \\
\hline 17 & $\mathrm{H}$ & -88.0350 & 29.1000 & 48.7660 & $\mathrm{H}$ \\
\hline \multicolumn{6}{|c|}{0.0000} \\
\hline 18 & $\mathrm{~S}$ & -90.8070 & 29.4110 & 48.2790 & S. 3 \\
\hline \multicolumn{6}{|c|}{0.5380} \\
\hline 19 & $\mathrm{C}$ & -92.3490 & 28.5520 & 48.0200 & C.ar \\
\hline \multicolumn{6}{|c|}{0.0070} \\
\hline 20 & 0 & -90.5480 & 29.4010 & 49.7030 & 0.3 \\
\hline \multicolumn{6}{|c|}{0.2150} \\
\hline 21 & 0 & -90.8740 & 30.6480 & 47.5340 & 0.3 \\
\hline \multicolumn{6}{|c|}{0.2150} \\
\hline 22 & $\mathrm{C}$ & -92.9200 & 27.8380 & 49.0780 & C.ar \\
\hline 0.1500 & & & & & \\
\hline 23 & $\mathrm{H}$ & -92.4710 & 27.8000 & 50.0650 & $\mathrm{H}$ \\
\hline 0.1500 & & & & & \\
\hline 24 & $\mathrm{C}$ & -94.0960 & 27.1670 & 48.8030 & C.ar \\
\hline 0.0860 & & & & & \\
\hline 25 & $\mathrm{C}$ & -94.6970 & 27.1590 & 47.5440 & C.ar \\
\hline 0.1170 & & & & & \\
\hline 26 & $\mathrm{C}$ & -94.1210 & 27.8410 & 46.4890 & C.ar \\
\hline 0.1500 & & & & & \\
\hline 27 & $\mathrm{H}$ & -94.5590 & 27.8400 & 45.4950 & $\mathrm{H}$ \\
\hline 0.1500 & & & & & \\
\hline 28 & $\mathrm{C}$ & -92.9310 & 28.5400 & 46.7310 & C.ar \\
\hline 0.1500 & & & & & \\
\hline 29 & $\mathrm{H}$ & -92.4520 & 29.0670 & 45.9050 & $\mathrm{H}$ \\
\hline 0.1500 & & & & & \\
\hline 30 & $\mathrm{C}$ & -94.9500 & 26.3520 & 49.6590 & C. 2 \\
\hline 0.4840 & & & & & \\
\hline 31 & 0 & -94.7750 & 26.0930 & 50.8370 & 0.2 \\
\hline 0.5700 & & & & & \\
\hline 32 & $\mathrm{C}$ & -96.1130 & 25.8640 & 48.8130 & C. 2 \\
\hline 0.6300 & & & & & \\
\hline 33 & 0 & -97.0240 & 25.1630 & 49.1950 & 0.2 \\
\hline 0.5700 & & & & & \\
\hline 34 & $\mathrm{~N}$ & -95.8730 & 26.3940 & 47.5540 & N.am \\
\hline 0.4770 & & & & & \\
\hline 35 & $\mathrm{C}$ & -96.7160 & 26.1830 & 46.4080 & C. 3 \\
\hline 0.3000 & & & & & \\
\hline 36 & $\mathrm{C}$ & -85.4150 & 32.0050 & 46.8200 & C.ar \\
\hline 0.1500 & & & & & \\
\hline $\begin{array}{r}37 \\
0.1500^{37}\end{array}$ & $\mathrm{H}$ & -85.6620 & 32.0870 & 47.8750 & $\mathrm{H}$ \\
\hline
\end{tabular}




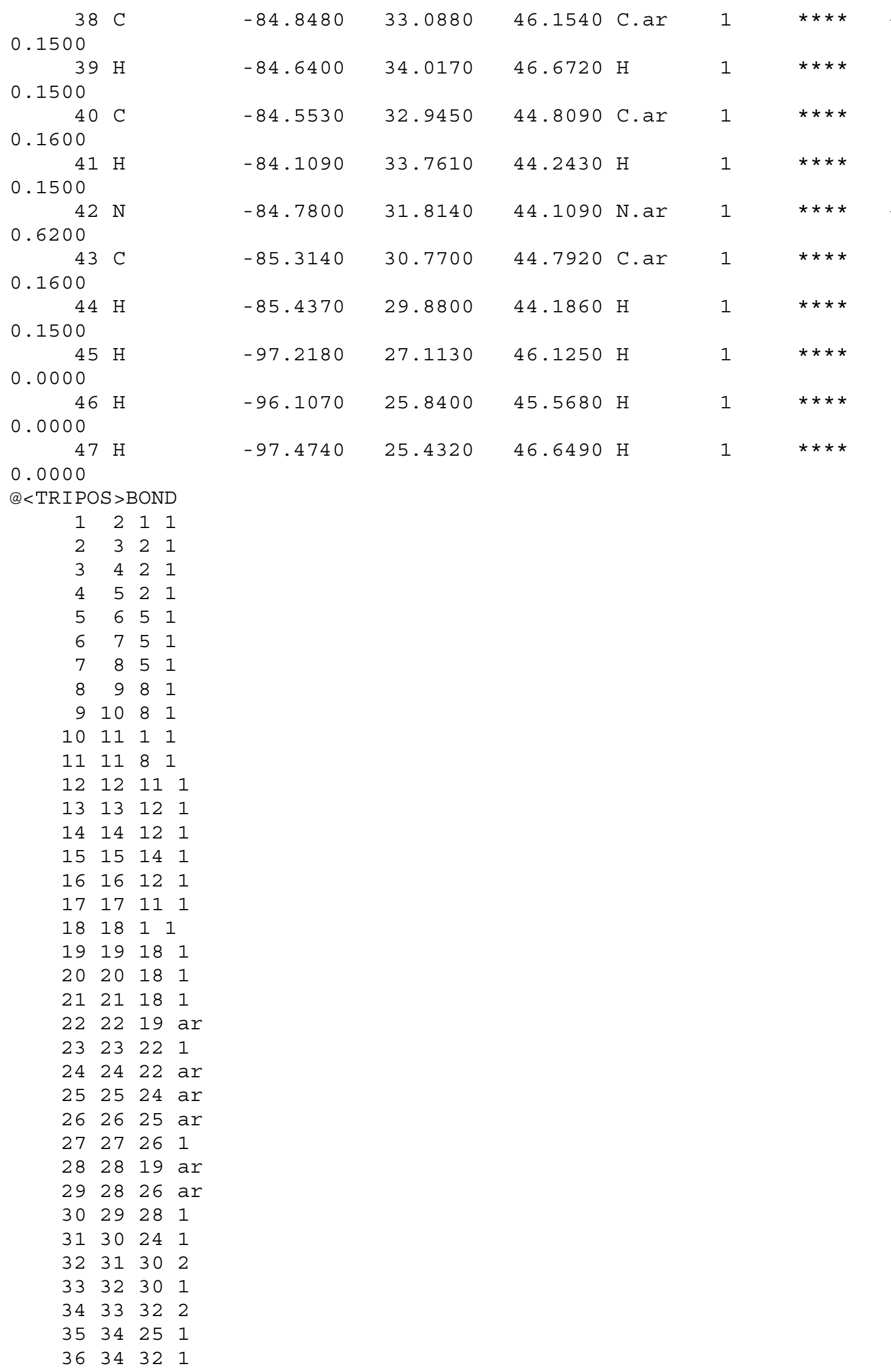




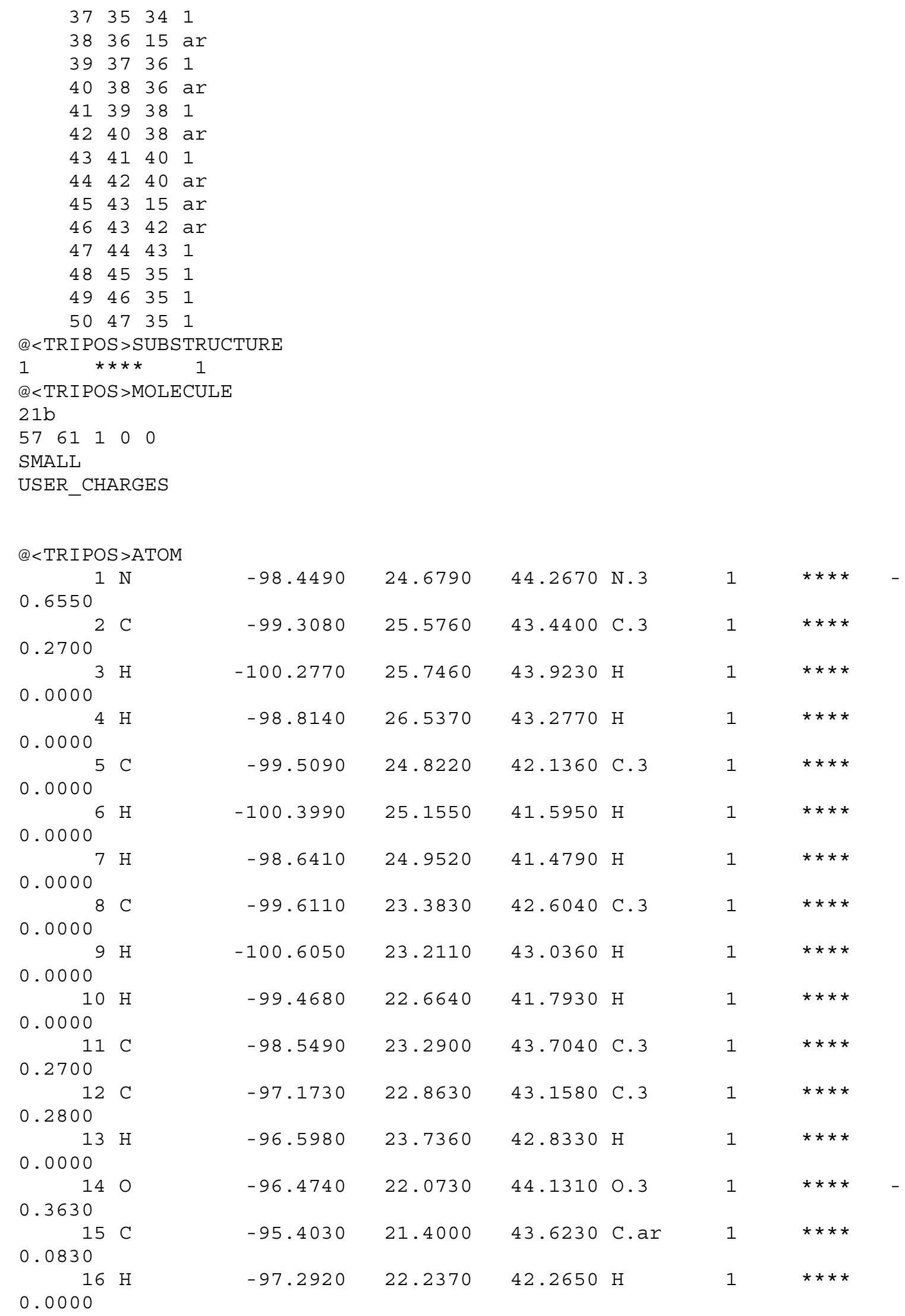




\begin{tabular}{|c|c|c|c|c|c|c|c|}
\hline $0.0000^{17}$ & $\mathrm{H}$ & -98.8870 & 22.5640 & 44.4500 & $\mathrm{H}$ & 1 & $\star \star \star *$ \\
\hline $0.5380^{18}$ & S & -98.7050 & 24.8490 & 45.9440 & S. 3 & 1 & $\star \star \star * *$ \\
\hline $\begin{array}{r}19 \\
0.0070^{19}\end{array}$ & $\mathrm{C}$ & -97.2700 & 25.7630 & 46.4600 & C.ar & 1 & 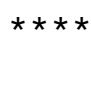 \\
\hline $0.2150^{20}$ & $\mathrm{O}$ & -99.8540 & 25.7080 & 46.1320 & 0.3 & 1 & $\star \star \star \star$ \\
\hline $0.2150^{21}$ & O & -98.6430 & 23.5290 & 46.5320 & 0.3 & 1 & 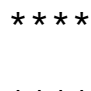 \\
\hline $0.1500^{22}$ & C & -96.8020 & 25.6360 & 47.7700 & C.ar & 1 & $\star \star \star \star *$ \\
\hline $0.1500^{23}$ & $\mathrm{H}$ & -97.2780 & 24.9920 & 48.5030 & $\mathrm{H}$ & 1 & $\star \star \star \star *$ \\
\hline $0.0860^{24}$ & $\mathrm{C}$ & -95.6790 & 26.3730 & 48.0920 & C.ar & 1 & $\star \star \star \star *$ \\
\hline $0.1170^{25}$ & $\mathrm{C}$ & -95.0250 & 27.2140 & 47.1900 & C.ar & 1 & $\star \star \star \star *$ \\
\hline $0.1500^{26}$ & $\mathrm{C}$ & -95.5010 & 27.3540 & 45.8990 & C.ar & 1 & $\star \star \star \star *$ \\
\hline $\begin{array}{r}27 \\
0.1500^{2}\end{array}$ & $\mathrm{H}$ & -95.0240 & 28.0060 & 45.1760 & $\mathrm{H}$ & 1 & 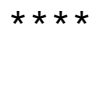 \\
\hline $\begin{array}{r}28 \\
0.1500^{2}\end{array}$ & $\mathrm{C}$ & -96.6400 & 26.6230 & 45.5340 & C.ar & 1 & $\star \star \star *$ \\
\hline $\begin{array}{r}29 \\
0.1500^{2}\end{array}$ & $\mathrm{H}$ & -97.0400 & 26.7190 & 44.5240 & $\mathrm{H}$ & 1 & 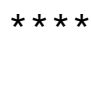 \\
\hline $\begin{array}{r}30 \\
0.4840^{3}\end{array}$ & $\mathrm{C}$ & -94.9340 & 26.4630 & 49.3410 & C. 2 & 1 & $\star \star \star \star *$ \\
\hline $0.5700^{31}$ & $\mathrm{O}$ & -95.1910 & 25.9130 & 50.3980 & 0.2 & 1 & 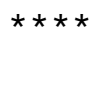 \\
\hline $0.6300^{32}$ & C & -93.7660 & 27.4010 & 49.1050 & C. 2 & 1 & $\star \star \star \star *$ \\
\hline $0.5700^{33}$ & O & -92.9310 & 27.7040 & 49.9300 & 0.2 & 1 & 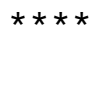 \\
\hline $\begin{array}{r}34 \\
0.4770^{3}\end{array}$ & $\mathrm{~N}$ & -93.9050 & 27.8140 & 47.7890 & N. am & 1 & $\star \star \star \star *$ \\
\hline $0.4440^{35}$ & C & -92.9980 & 28.7340 & 47.1440 & C. 3 & 1 & $\star \star \star \star$ \\
\hline $0.1500^{36}$ & $\mathrm{C}$ & -95.6220 & 20.1350 & 43.0920 & C.ar & 1 & $\star \star \star \star$ \\
\hline $0.1500^{37}$ & $\mathrm{H}$ & -96.6130 & 19.6940 & 43.1110 & $\mathrm{H}$ & 1 & 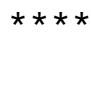 \\
\hline $0.1500^{38}$ & $\mathrm{C}$ & -94.5560 & 19.4330 & 42.5360 & C.ar & 1 & $\star \star \star \star *$ \\
\hline $0.1500^{39}$ & $\mathrm{H}$ & -94.6930 & 18.4390 & 42.1250 & $\mathrm{H}$ & 1 & $\star \star \star \star$ \\
\hline $\begin{array}{r}40 \\
0.1600^{4}\end{array}$ & $\mathrm{C}$ & -93.3080 & 20.0340 & 42.5200 & C.ar & 1 & 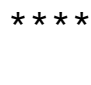 \\
\hline $0.1500^{41}$ & $\mathrm{H}$ & -92.4480 & 19.5200 & 42.0990 & $\mathrm{H}$ & 1 & $\star \star \star \star *$ \\
\hline $0.6200^{42}$ & $\mathrm{~N}$ & -93.0620 & 21.2670 & 43.0210 & N.ar & 1 & $\star \star \star \star *$ \\
\hline $0.1600^{43}$ & $\mathrm{C}$ & -94.1240 & 21.9320 & 43.5340 & C.ar & 1 & $\star \star \star \star *$ \\
\hline $0.1500^{44}$ & $\mathrm{H}$ & -93.9050 & 22.9400 & 43.8710 & $\mathrm{H}$ & 1 & $\star \star \star \star$ \\
\hline
\end{tabular}




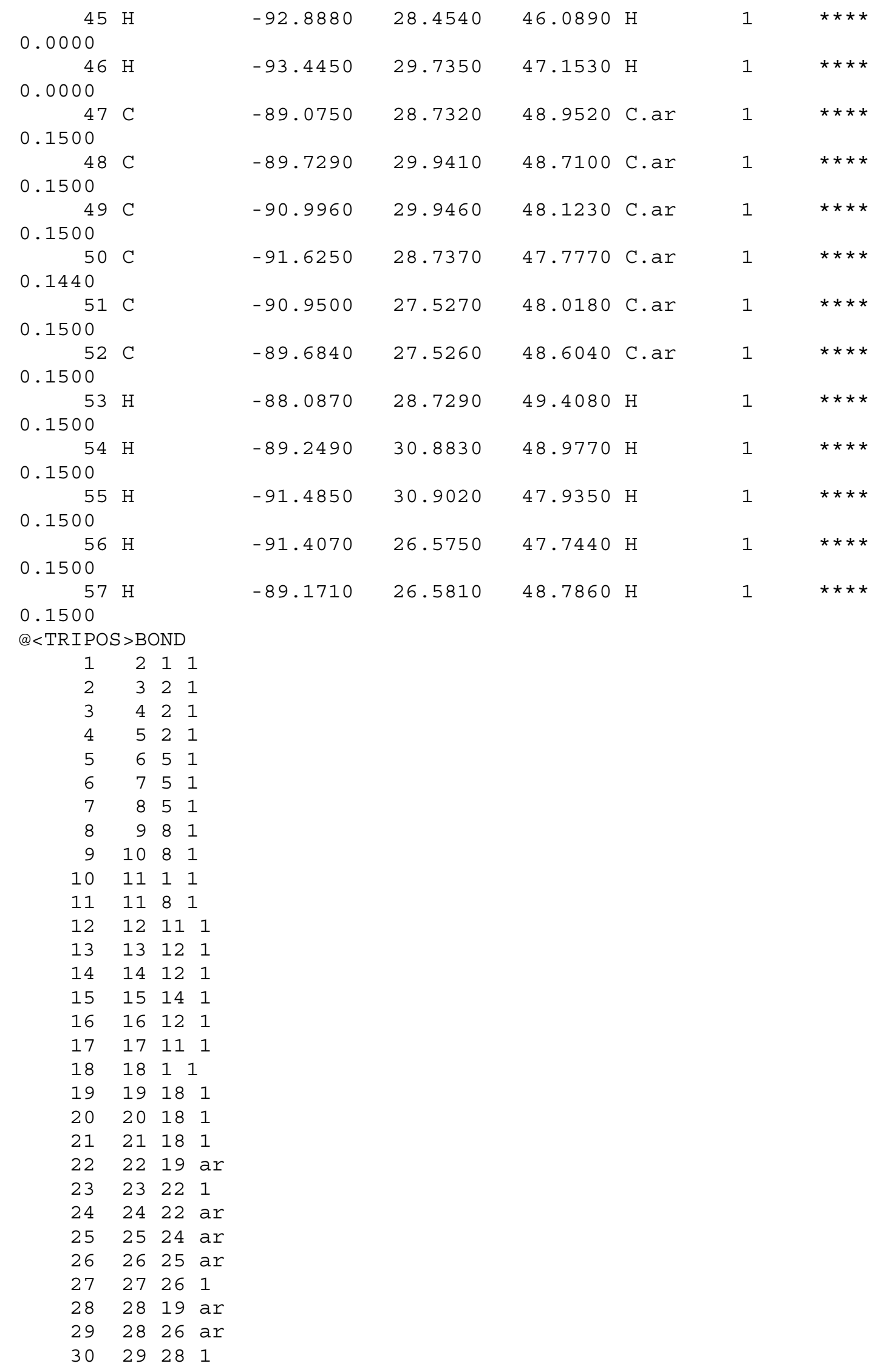




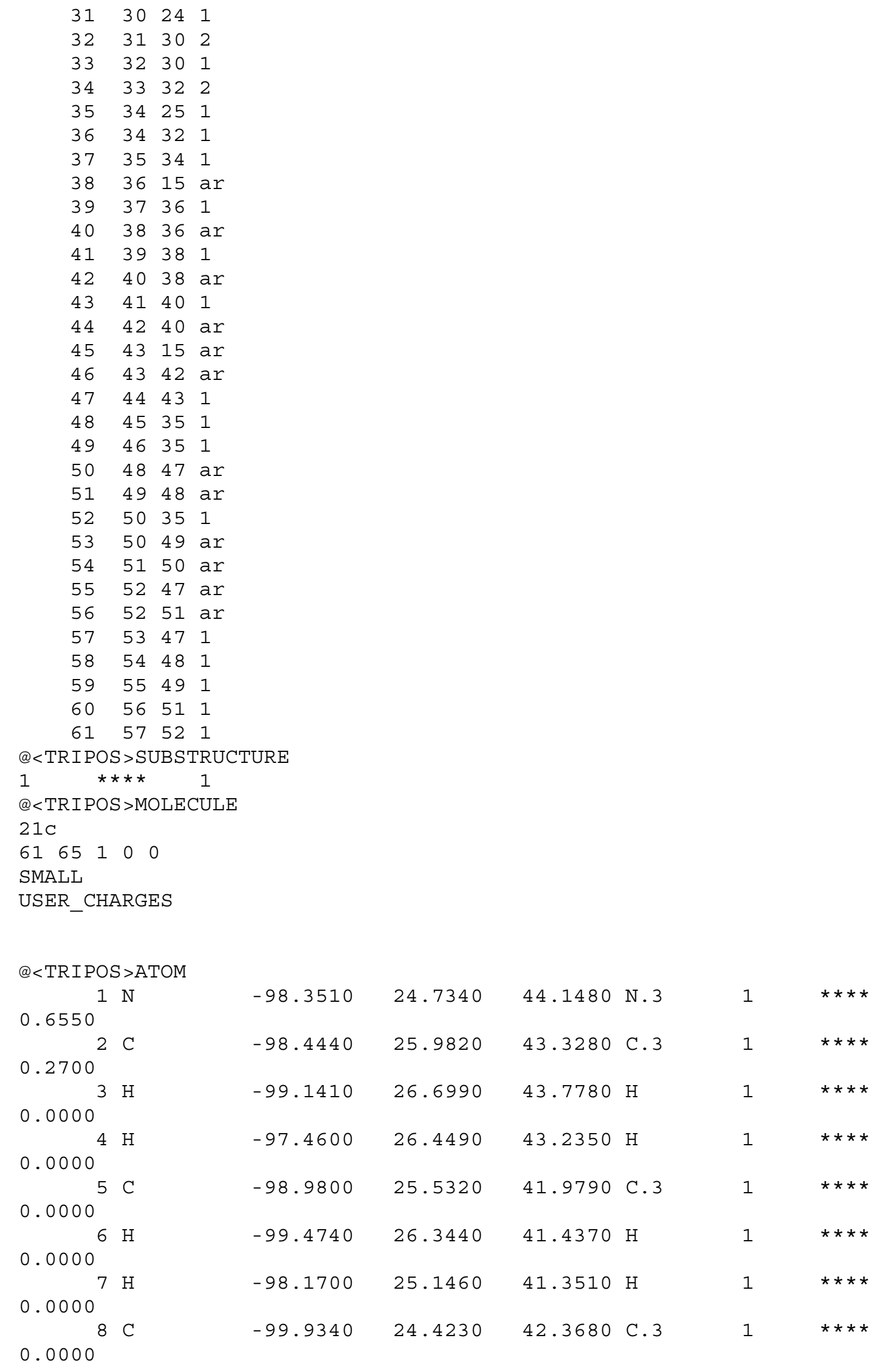




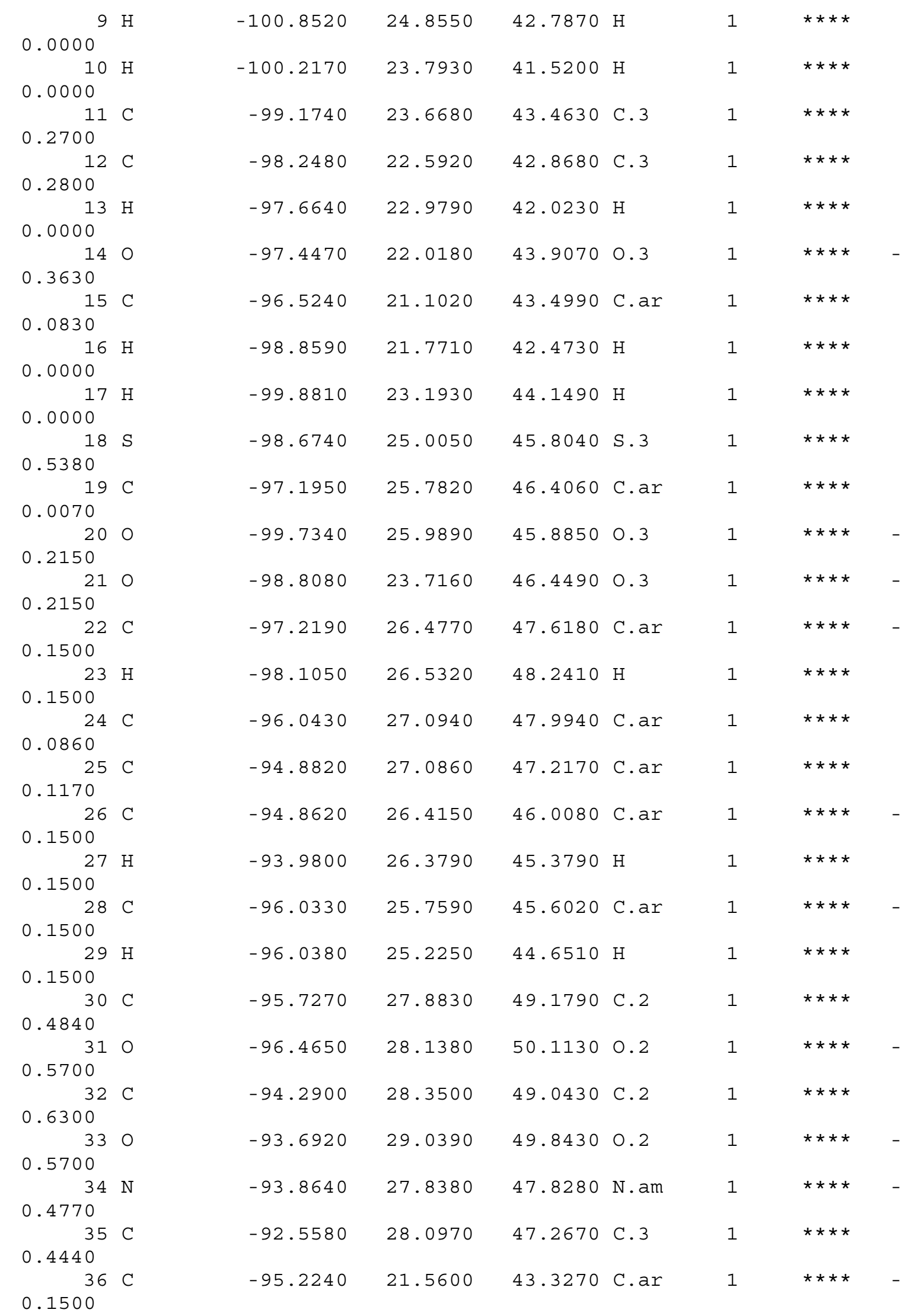




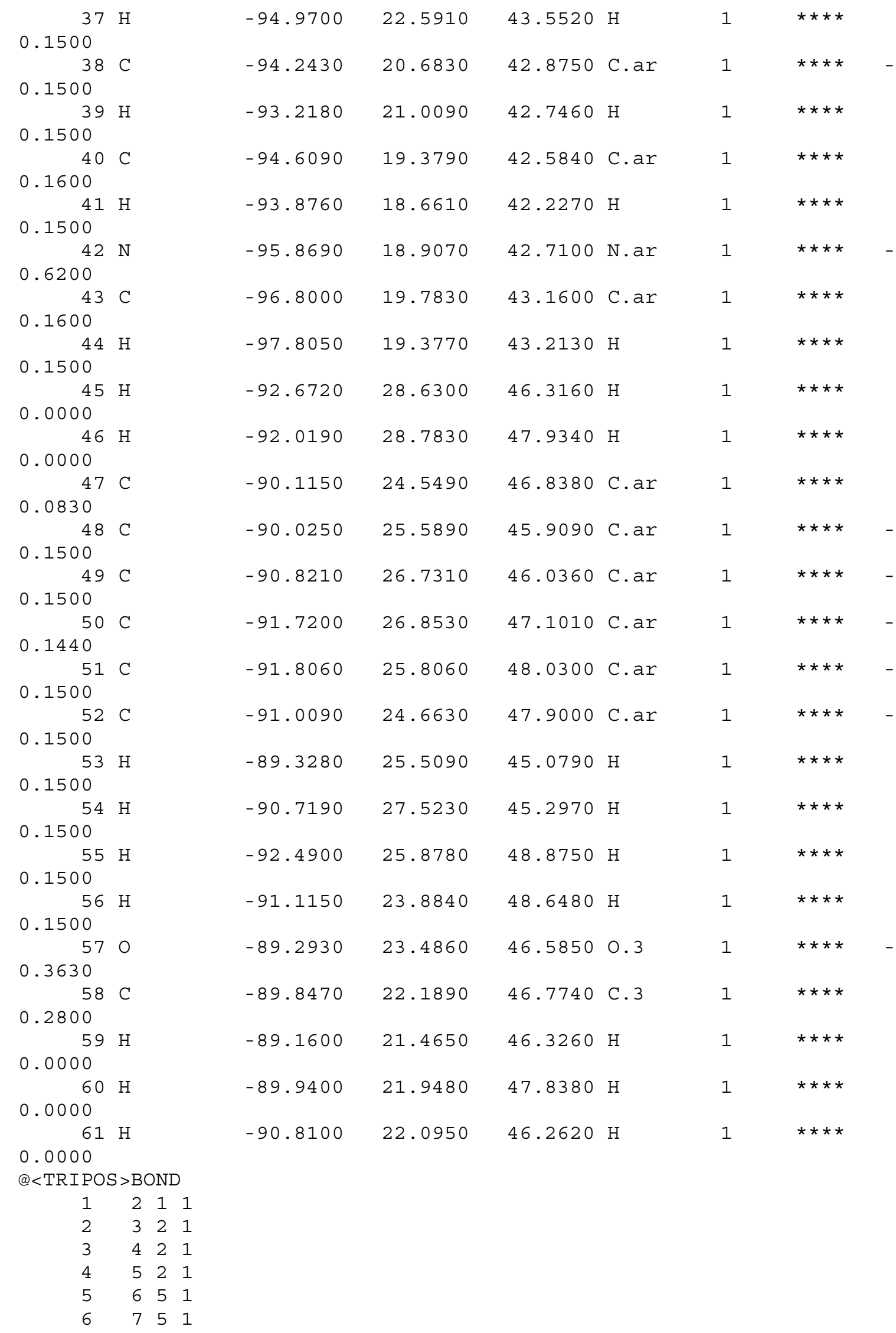




\begin{tabular}{|c|c|c|c|}
\hline 7 & 8 & 51 & 1 \\
\hline 8 & 9 & 81 & 1 \\
\hline 9 & 10 & 81 & 1 \\
\hline 10 & 11 & 11 & 1 \\
\hline 11 & 11 & 81 & 1 \\
\hline 12 & 12 & 11 & 1 \\
\hline 13 & 13 & 12 & 1 \\
\hline 14 & 14 & 12 & 1 \\
\hline 15 & 15 & 14 & 1 \\
\hline 16 & 16 & 12 & 1 \\
\hline 17 & 17 & 11 & 1 \\
\hline 18 & 18 & & 1 \\
\hline 19 & 19 & 18 & 1 \\
\hline 20 & 20 & 18 & 1 \\
\hline 21 & 21 & 18 & 1 \\
\hline 22 & 22 & 19 & $\operatorname{ar}$ \\
\hline 23 & 23 & 22 & 1 \\
\hline 24 & 24 & 22 & $\operatorname{ar}$ \\
\hline 25 & 25 & 24 & $\operatorname{ar}$ \\
\hline 26 & 26 & 25 & $\operatorname{ar}$ \\
\hline 27 & 27 & 26 & 1 \\
\hline 28 & 28 & 19 & $\operatorname{ar}$ \\
\hline 29 & 28 & 26 & $\operatorname{ar}$ \\
\hline 30 & 29 & 28 & 1 \\
\hline 31 & 30 & 24 & 1 \\
\hline 32 & 31 & 30 & 2 \\
\hline 33 & 32 & 30 & 1 \\
\hline 34 & 33 & 32 & 2 \\
\hline 35 & 34 & 25 & 1 \\
\hline 36 & 34 & 32 & 1 \\
\hline 37 & 35 & 34 & 1 \\
\hline 38 & 36 & 15 & $\operatorname{ar}$ \\
\hline 39 & 37 & 36 & 1 \\
\hline 40 & 38 & 36 & $\operatorname{ar}$ \\
\hline 41 & 39 & 38 & 1 \\
\hline 42 & 40 & 38 & $\operatorname{ar}$ \\
\hline 43 & 41 & 40 & 1 \\
\hline 44 & 42 & 40 & $\operatorname{ar}$ \\
\hline 45 & 43 & 15 & $a r$ \\
\hline 46 & 43 & 42 & $\operatorname{ar}$ \\
\hline 47 & 44 & 43 & 1 \\
\hline 48 & 45 & 35 & 1 \\
\hline 49 & 46 & 35 & 1 \\
\hline 50 & 48 & 47 & $\operatorname{ar}$ \\
\hline 51 & 49 & 48 & $\operatorname{ar}$ \\
\hline 52 & 50 & 35 & 1 \\
\hline 53 & 50 & 49 & ar \\
\hline 54 & 51 & 50 & $\operatorname{ar}$ \\
\hline 55 & 52 & 47 & $a r$ \\
\hline 56 & 52 & 51 & $\operatorname{ar}$ \\
\hline 57 & 53 & 48 & 1 \\
\hline 58 & 54 & 49 & 1 \\
\hline 59 & 55 & 51 & 1 \\
\hline 60 & 56 & 52 & 1 \\
\hline 61 & 57 & 47 & 1 \\
\hline 62 & 58 & 57 & 1 \\
\hline 63 & 59 & 58 & 1 \\
\hline
\end{tabular}




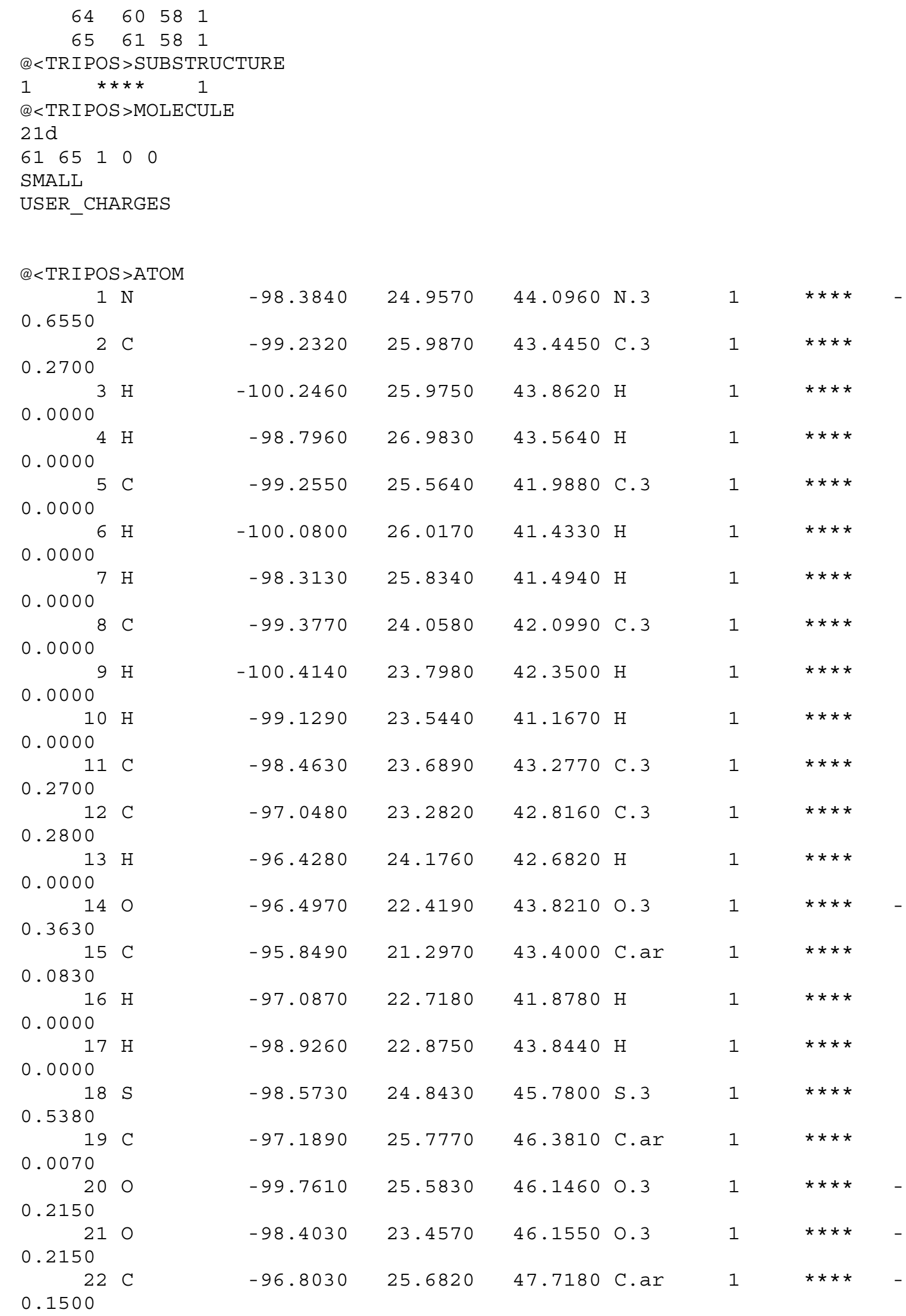




\begin{tabular}{|c|c|c|c|c|c|c|c|c|}
\hline 23 & $\mathrm{H}$ & -97.3250 & 25.0550 & 48.4340 & $\mathrm{H}$ & 1 & $\star \star \star \star$ & \\
\hline 0.1500 & & & & & & & & \\
\hline 24 & $\mathrm{C}$ & -95.6740 & 26.3920 & 48.0770 & C.ar & 1 & $\star \star \star \star$ & \\
\hline 0.0860 & & & & & & & & \\
\hline 25 & $\mathrm{C}$ & -94.9430 & 27.1810 & 47.1840 & C.ar & 1 & $\star \star \star \star$ & \\
\hline 0.1170 & & & & & & & & \\
\hline 26 & $\mathrm{C}$ & -95.3520 & 27.3100 & 45.8690 & C.ar & 1 & 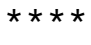 & - \\
\hline 0.1500 & & & & & & & & \\
\hline 27 & $\mathrm{H}$ & -94.8130 & 27.9170 & 45.1530 & $\mathrm{H}$ & 1 & $\star \star \star \star$ & \\
\hline 0.1500 & & & & & & & & \\
\hline 28 & $\mathrm{C}$ & -96.4900 & 26.5990 & 45.4670 & C.ar & 1 & 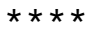 & - \\
\hline 0.1500 & & & & & & & & \\
\hline 29 & $\mathrm{H}$ & -96.8290 & 26.6680 & 44.4340 & $\mathrm{H}$ & 1 & $\star \star \star \star$ & \\
\hline 0.1500 & & & & & & & & \\
\hline 30 & $\mathrm{C}$ & -94.9600 & 26.4600 & 49.3460 & C. 2 & 1 & 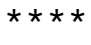 & \\
\hline 0.4840 & & & & & & & & \\
\hline 31 & 0 & -95.2720 & 25.9240 & 50.3950 & 0.2 & 1 & $\star \star \star \star ~$ & - \\
\hline 0.5700 & & & & & & & & \\
\hline 32 & $\mathrm{C}$ & -93.7330 & 27.3270 & 49.1310 & C. 2 & 1 & $\star \star \star \star$ & \\
\hline 0.6300 & & & & & & & & \\
\hline 33 & O & -92.8920 & 27.5830 & 49.9680 & 0.2 & 1 & $\star \star \star \star \star$ & - \\
\hline 0.5700 & & & & & & & & \\
\hline 34 & $\mathrm{~N}$ & -93.8080 & 27.7260 & 47.8080 & N.am & 1 & $\star \star \star \star$ & - \\
\hline 0.4770 & & & & & & & & \\
\hline 35 & $\mathrm{C}$ & -92.8170 & 28.5580 & 47.1650 & C. 3 & 1 & $\star \star \star \star \star$ & \\
\hline 0.4440 & & & & & & & & \\
\hline 36 & $\mathrm{C}$ & -94.4770 & 21.4340 & 43.2060 & C.ar & 1 & $\star \star \star \star$ & - \\
\hline 0.1500 & & & & & & & & \\
\hline 37 & $\mathrm{H}$ & -93.9820 & 22.3750 & 43.4360 & $\mathrm{H}$ & 1 & $\star \star \star * *$ & \\
\hline 0.1500 & & & & & & & & \\
\hline 38 & $\mathrm{C}$ & -93.7330 & 20.3650 & 42.7150 & C.ar & 1 & $\star \star \star \star *$ & - \\
\hline 0.1500 & & & & & & & & \\
\hline 39 & $\mathrm{H}$ & -92.6630 & 20.4540 & 42.5580 & $\mathrm{H}$ & 1 & $\star \star \star \star *$ & \\
\hline 0.1500 & & & & & & & & \\
\hline 40 & $\mathrm{C}$ & -94.3960 & 19.1850 & 42.4280 & C.ar & 1 & 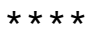 & \\
\hline 0.1600 & & & & & & & & \\
\hline 41 & $\mathrm{H}$ & -93.8610 & 18.3210 & 42.0440 & $\mathrm{H}$ & 1 & $\star \star \star \star *$ & \\
\hline 0.1500 & & & & & & & & \\
\hline 42 & $\mathrm{~N}$ & -95.7270 & 19.0160 & 42.5930 & N.ar & 1 & $\star \star \star \star *$ & - \\
\hline 0.6200 & & & & & & & & \\
\hline 43 & $\mathrm{C}$ & -96.4220 & 20.0740 & 43.0810 & C.ar & 1 & 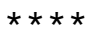 & \\
\hline 0.1600 & & & & & & & & \\
\hline 44 & $\mathrm{H}$ & -97.4840 & 19.8840 & 43.1790 & $\mathrm{H}$ & 1 & 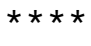 & \\
\hline 0.1500 & & & & & & & & \\
\hline 45 & $\mathrm{H}$ & -92.9070 & 28.4530 & 46.0770 & $\mathrm{H}$ & 1 & $\star \star \star \star$ & \\
\hline 0.0000 & & & & & & & & \\
\hline 46 & $\mathrm{H}$ & -93.0400 & 29.6080 & 47.3940 & $\mathrm{H}$ & 1 & $\star \star \star \star ~$ & \\
\hline 0.0000 & & & & & & & & \\
\hline 47 & $\mathrm{C}$ & -88.7740 & 27.5210 & 48.3020 & C.ar & 1 & $\star \star \star \star$ & \\
\hline 0.1010 & & & & & & & & \\
\hline 48 & $\mathrm{C}$ & -89.5440 & 26.6300 & 47.5510 & C.ar & 1 & $\star \star \star \star \star$ & - \\
\hline 0.1500 & & & & & & & & \\
\hline 49 & $\mathrm{C}$ & -90.8520 & 26.9630 & 47.1860 & C.ar & 1 & 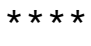 & - \\
\hline 0.1500 & & & & & & & & \\
\hline $\begin{array}{r}50 \\
0.1440\end{array}$ & C & -91.4040 & 28.1960 & 47.5580 & C.ar & 1 & $\star \star \star \star *$ & - \\
\hline
\end{tabular}




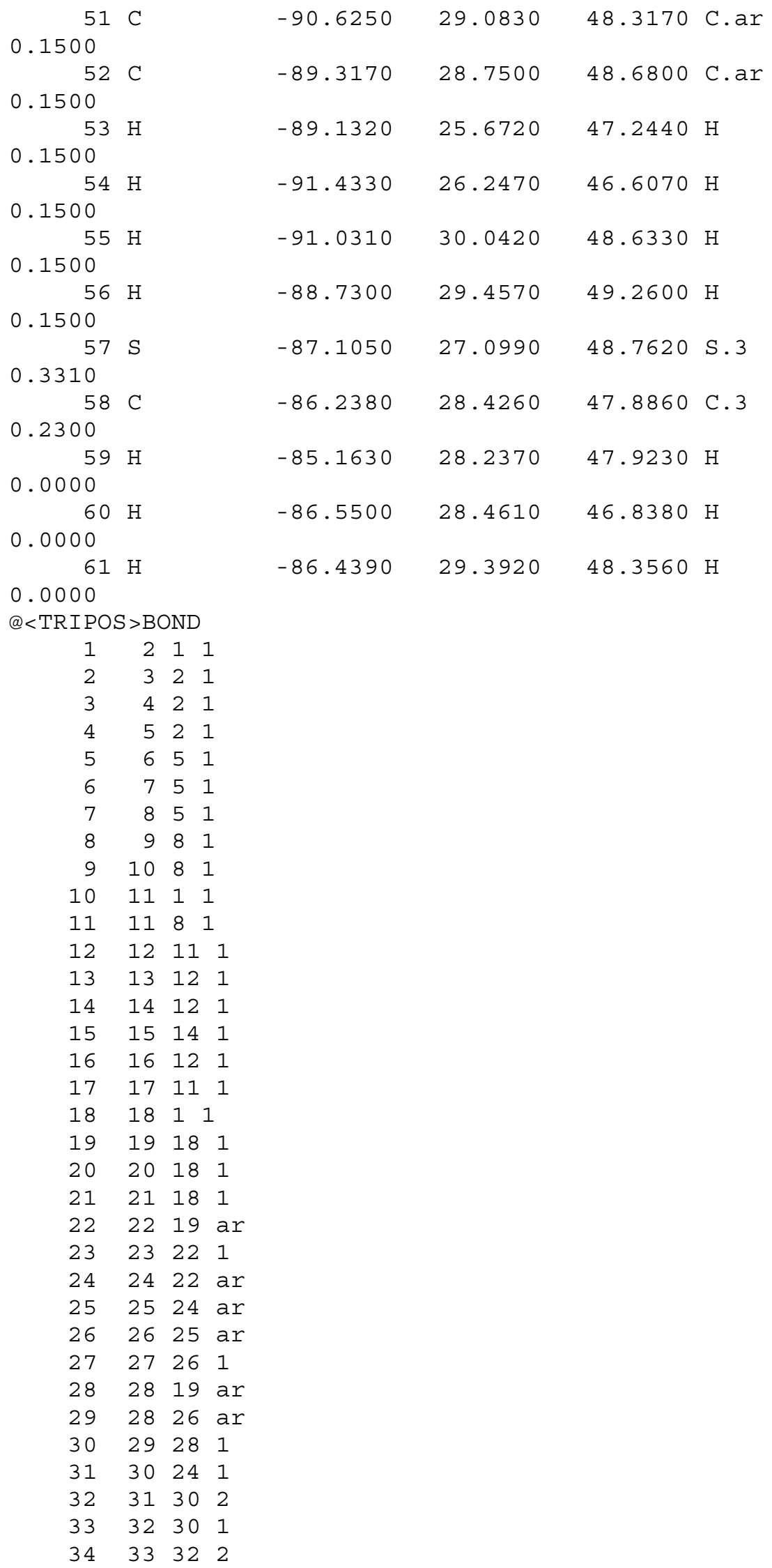




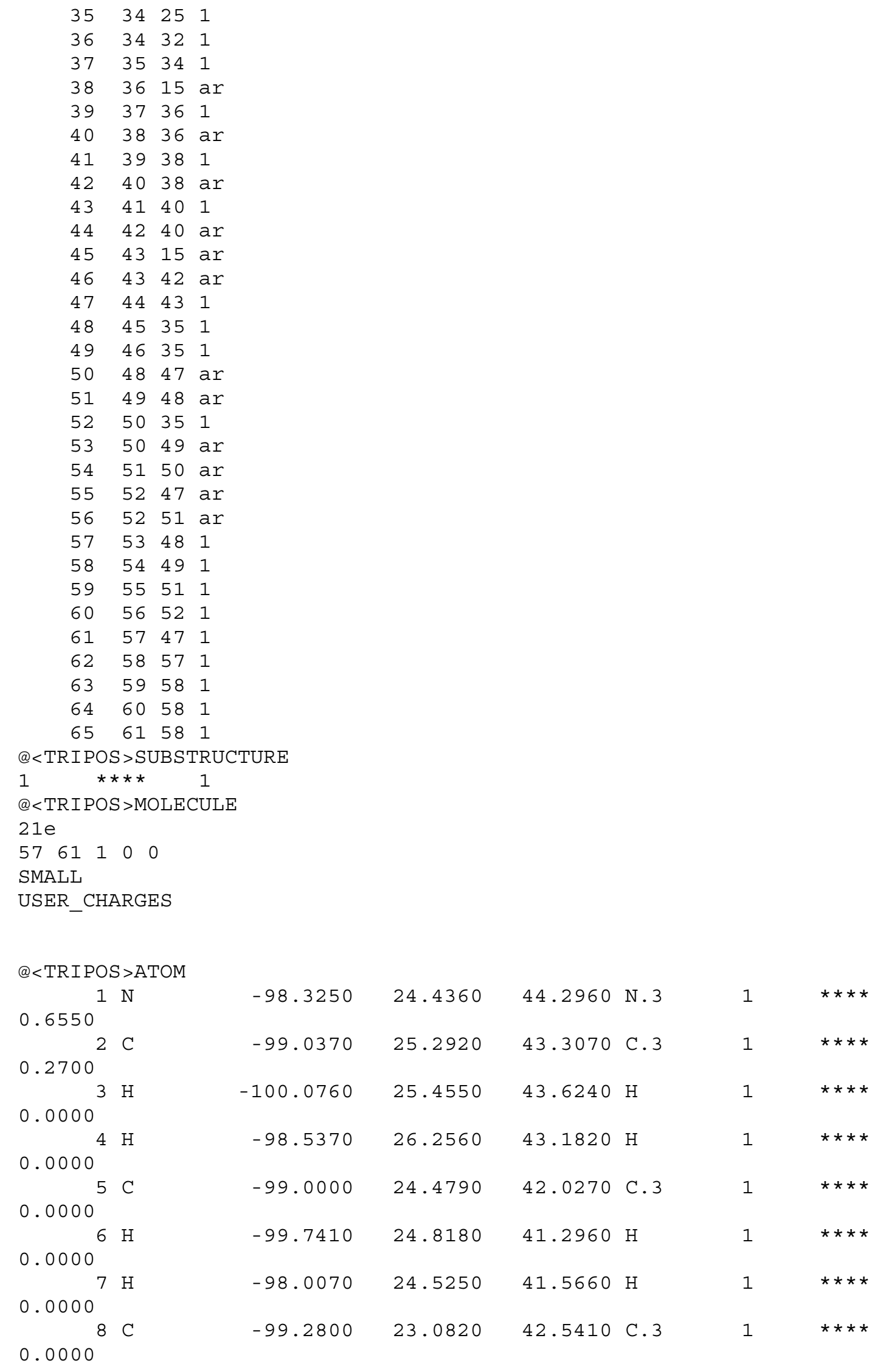




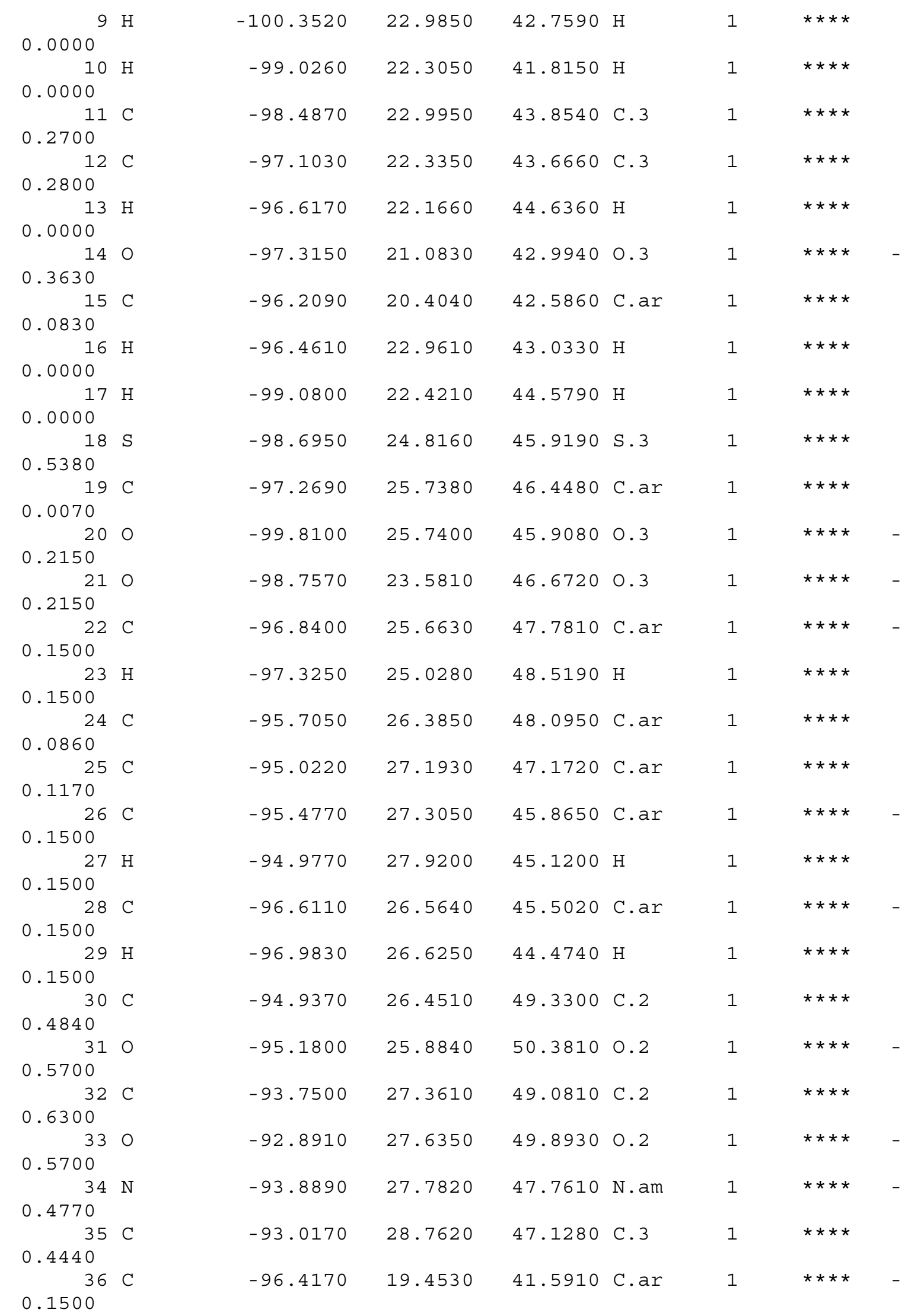




\begin{tabular}{|c|c|c|c|c|c|c|c|c|}
\hline \multirow{3}{*}{$\begin{array}{r}37 \\
0.1500 \\
38\end{array}$} & \multicolumn{2}{|l|}{$\mathrm{H}$} & \multirow[t]{2}{*}{-97.4040} & \multirow[t]{2}{*}{19.3210} & \multirow[t]{2}{*}{41.1540} & \multirow[t]{2}{*}{$\mathrm{H}$} & \multirow[t]{2}{*}{1} & \multirow[t]{2}{*}{$\star \star \star \star *$} \\
\hline & & & & & & & & \\
\hline & \multicolumn{2}{|l|}{$\mathrm{C}$} & -95.3580 & 18.6680 & 41.1450 & C.ar & 1 & $\star \star \star \star *$ \\
\hline \multicolumn{9}{|c|}{0.1500} \\
\hline 39 & \multicolumn{2}{|l|}{$\mathrm{H}$} & -95.5060 & 17.9220 & 40.3720 & $\mathrm{H}$ & 1 & $\star \star \star \star \star$ \\
\hline \multicolumn{9}{|c|}{0.1500} \\
\hline 40 & \multicolumn{2}{|l|}{ C } & -94.1150 & 18.8680 & 41.7170 & C.ar & 1 & $\star \star \star \star$ \\
\hline \multicolumn{9}{|c|}{0.1600} \\
\hline 41 & \multicolumn{2}{|l|}{$\mathrm{H}$} & -93.2560 & 18.2830 & 41.4020 & $\mathrm{H}$ & 1 & $\star \star \star \star \star$ \\
\hline \multicolumn{9}{|c|}{0.1500} \\
\hline 42 & $\mathrm{~N}$ & & -93.8750 & 19.7760 & 42.6870 & N.ar & 1 & 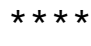 \\
\hline 0.6200 & & & & & & & & \\
\hline 43 & $\mathrm{C}$ & & -94.9290 & 20.5240 & 43.1000 & C.ar & 1 & $\star \star \star \star \star$ \\
\hline 0.1600 & & & & & & & & \\
\hline 44 & $\mathrm{H}$ & & -94.6880 & 21.2180 & 43.8960 & $\mathrm{H}$ & 1 & $\star \star \star \star$ \\
\hline 0.1500 & & & & & & & & \\
\hline 45 & $\mathrm{H}$ & & -92.9730 & 28.5520 & 46.0490 & $\mathrm{H}$ & 1 & $\star \star \star \star \star$ \\
\hline 0.0000 & & & & & & & & \\
\hline 46 & $\mathrm{H}$ & & -93.4330 & 29.7730 & 47.2240 & $\mathrm{H}$ & 1 & $\star \star \star \star \star$ \\
\hline 0.0000 & & & & & & & & \\
\hline 47 & $\mathrm{C}$ & & -88.9500 & 28.6420 & 48.5780 & C.ar & 1 & $\star \star \star \star$ \\
\hline 0.1900 & & & & & & & & \\
\hline 48 & $\mathrm{C}$ & & -89.5950 & 27.4580 & 48.2570 & C.ar & 1 & 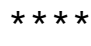 \\
\hline 0.1500 & & & & & & & & \\
\hline 49 & $\mathrm{C}$ & & -90.9260 & 27.4830 & 47.8190 & C.ar & 1 & $\star \star \star \star$ \\
\hline 0.1500 & & & & & & & & \\
\hline 50 & $\mathrm{C}$ & & -91.6080 & 28.6920 & 47.7050 & C.ar & 1 & 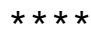 \\
\hline 0.1440 & & & & & & & & \\
\hline 51 & $\mathrm{C}$ & & -90.9380 & 29.8740 & 48.0540 & C.ar & 1 & $\star \star \star \star$ \\
\hline 0.1500 & & & & & & & & \\
\hline 52 & $\mathrm{C}$ & & -89.6080 & 29.8470 & 48.4880 & C.ar & 1 & $\star \star \star \star$ \\
\hline 0.1500 & & & & & & & & \\
\hline 53 & $F$ & & -87.6680 & 28.6130 & 48.9740 & $F$ & 1 & 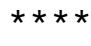 \\
\hline 0.1900 & & & & & & & & \\
\hline 54 & $\mathrm{H}$ & & -89.0560 & 26.5280 & 48.3350 & $\mathrm{H}$ & 1 & $\star \star \star \star *$ \\
\hline 0.1500 & & & & & & & & \\
\hline 55 & $\mathrm{H}$ & & -91.4100 & 26.5470 & 47.5650 & $\mathrm{H}$ & 1 & $\star \star \star \star$ \\
\hline 0.1500 & & & & & & & & \\
\hline 56 & $\mathrm{H}$ & & -91.4440 & 30.8290 & 47.9730 & $\mathrm{H}$ & 1 & 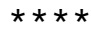 \\
\hline 0.1500 & & & & & & & & \\
\hline 57 & $\mathrm{H}$ & & -89.0910 & 30.7670 & 48.7350 & $\mathrm{H}$ & 1 & $\star \star \star \star$ \\
\hline 0.1500 & & & & & & & & \\
\hline @<TRIPOS & $5>\mathrm{BO}$ & $\mathrm{ND}$ & & & & & & \\
\hline 1 & 2 & 11 & & & & & & \\
\hline 2 & 3 & 21 & & & & & & \\
\hline 3 & 4 & 21 & & & & & & \\
\hline 4 & 5 & 21 & & & & & & \\
\hline 5 & 6 & 51 & & & & & & \\
\hline 6 & 7 & 51 & & & & & & \\
\hline 7 & 8 & 51 & & & & & & \\
\hline 8 & 9 & 81 & & & & & & \\
\hline 9 & 10 & 81 & & & & & & \\
\hline 10 & 11 & 11 & & & & & & \\
\hline 11 & 11 & 81 & & & & & & \\
\hline 12 & 12 & 111 & & & & & & \\
\hline 13 & 13 & 121 & & & & & & \\
\hline 14 & 14 & 121 & & & & & & \\
\hline
\end{tabular}




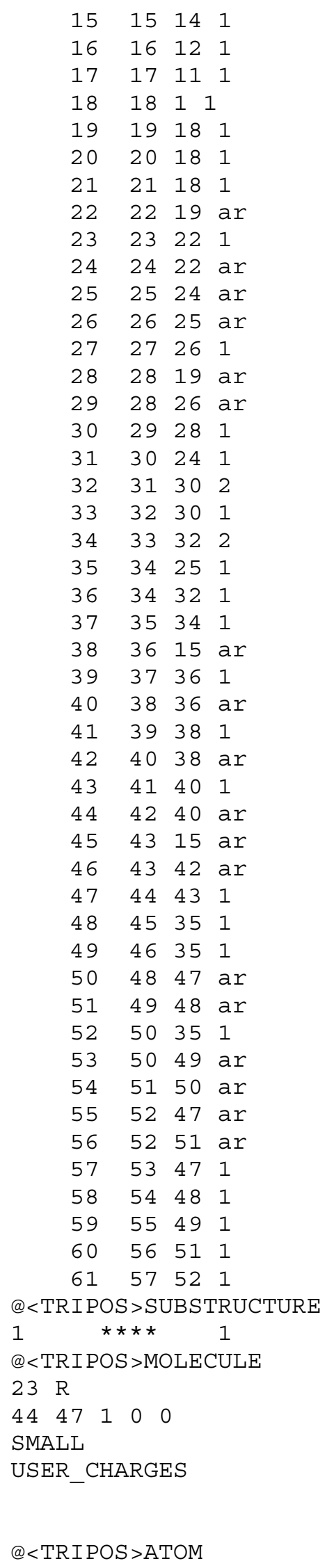




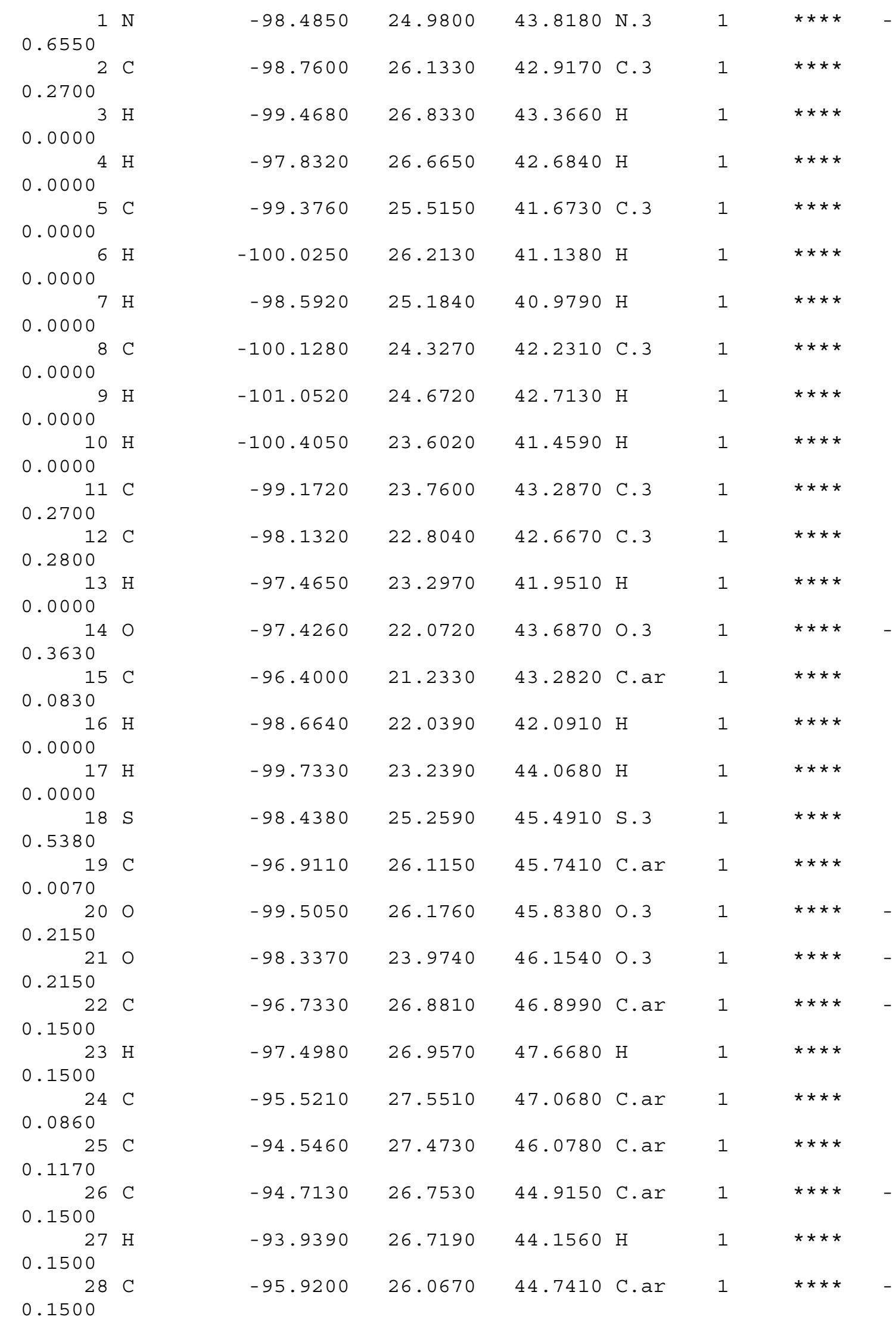




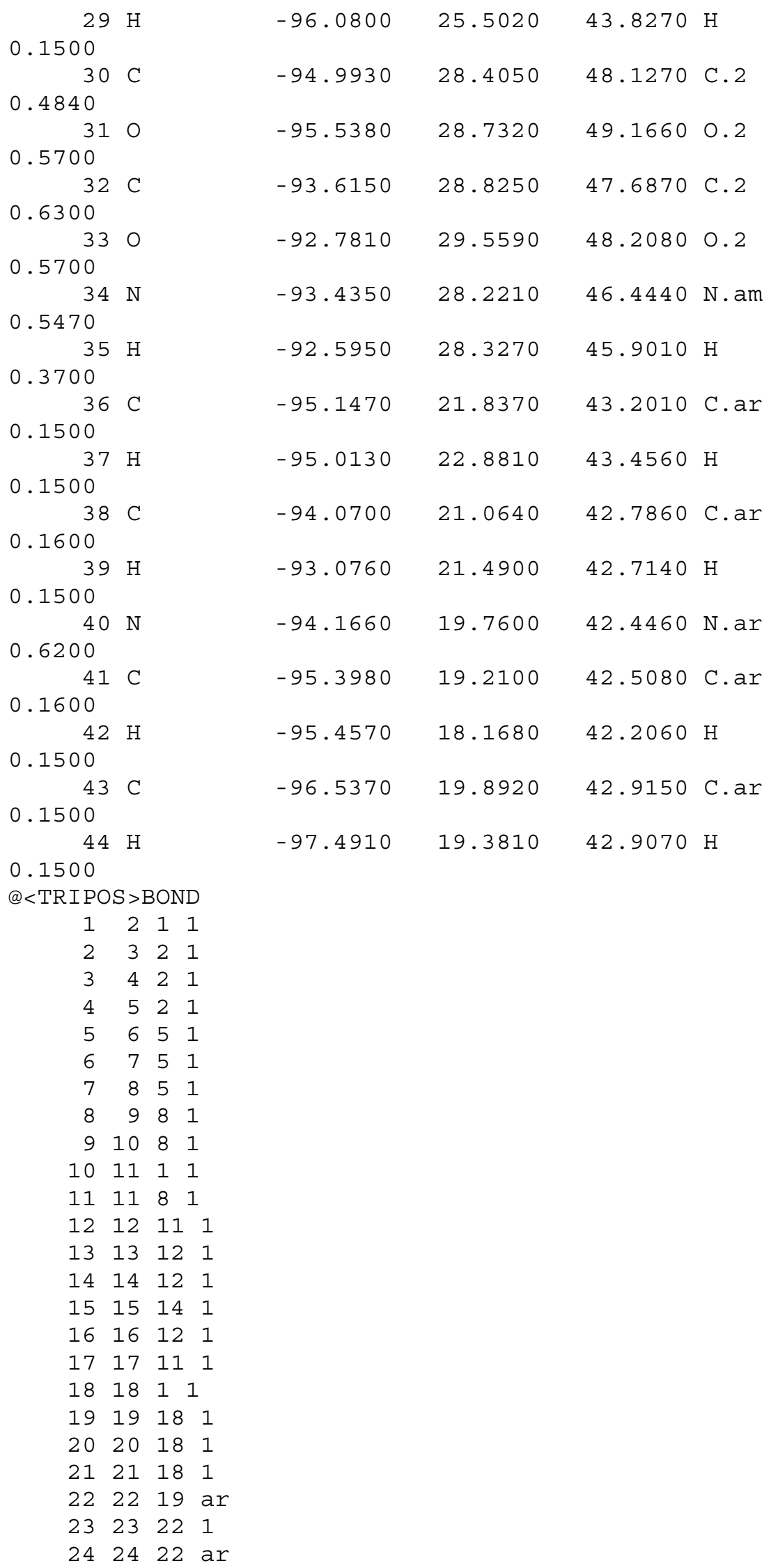




\begin{tabular}{|c|c|c|c|}
\hline 25 & 25 & 24 & ar \\
\hline 26 & 26 & 25 & ar \\
\hline 27 & 27 & 26 & 1 \\
\hline 28 & 28 & 19 & ar \\
\hline 29 & 28 & 26 & ar \\
\hline 30 & 29 & 28 & 1 \\
\hline 31 & 30 & 24 & 1 \\
\hline 32 & 31 & 30 & 2 \\
\hline 33 & 32 & 30 & 1 \\
\hline 34 & 33 & 32 & 2 \\
\hline 35 & 34 & 25 & 1 \\
\hline 36 & 34 & 32 & 1 \\
\hline 37 & 35 & 34 & 1 \\
\hline 38 & 36 & 15 & ar \\
\hline 39 & 37 & 36 & 1 \\
\hline 40 & 38 & 36 & ar \\
\hline 41 & 39 & 38 & 1 \\
\hline 42 & 40 & 38 & ar \\
\hline 43 & 41 & 40 & ar \\
\hline 44 & 42 & 41 & 1 \\
\hline 45 & 43 & 15 & ar \\
\hline 46 & 43 & 41 & ar \\
\hline 47 & 44 & 43 & 1 \\
\hline$@<$ TRIPC & $O S>S$ & SUB & STR \\
\hline $1+$ & $\star \star \star \star *$ & & 1 \\
\hline
\end{tabular}

